\title{
THE ORDER AND SYMBOL OF A DISTRIBUTION
}

BY

\author{
ALAN WEINSTEIN
}

\begin{abstract}
A definition is given, for an arbitrary distribution $g$ on a manifold $X$, of the order and symbol of $g$ at a point $(x, \xi)$ of the cotangent bundle $T^{*} X$.

If $X=\mathbf{R}^{n}$, the order of $g$ at $(0, \xi)$ is the growth order as $\tau \rightarrow \infty$ of the distributions

$$
g^{\tau}(x)=e^{-i \sqrt{\tau}\langle x, \xi\rangle} g(x / \sqrt{\tau}) ;
$$

if the order is less than or equal to $N$, the $N$-symbol of $g$ is the family $g^{T}$ modulo $O\left(\tau^{N-1 / 2}\right)$.

It is shown that the order and symbol behave in a simple way when $g$ is acted upon by a pseudo-differential operator. If $g$ is a Fourier integral distribution, suitable identifications can be made so that the symbol defined here agrees with the bundle-valued symbol defined by Hörmander.
\end{abstract}

\section{PREFACE}

Since the introduction of pseudo-differential operators, the analysis of distributions on a manifold $X$ has involved the geometry of the cotangent bundle $T^{*} X$. With the notion of wavefront set [6], one can localize a distribution at a nonzero cotangent vector $\xi$ to obtain its "microgerm", just as one localizes at $x \in X$ to obtain the ordinary germ. On the base space, $X$, one can go beyond the local level to the infinitesimal one; for a $C^{\infty}$ function, the result is its "jet", which can be thought of as a function on the tangent space $T_{x} X$. In this paper, we describe an analogous procedure for distributions: given a distribution $g$ on $X$ and a cotangent vector $\xi$, we construct a jet-like object called its symbol, which is a distribution on $T_{x} X$ depending on certain parameters. (In [13], we show that the symbol may be thought of as an object on $T_{\xi} T^{*} X$, thus completing the analogy with jets.)

In Chapter I, we define the symbol and the space in which it lies, we prove the elementary properties of the symbol, and we give some examples. Chapter II, written in collaboration with K. Sklower, establishes the relation between our symbol and the wavefront set. In Chapter III, we show that our symbol construction contains the one given by Hörmander [6] for a very special class of distributions-the Fourier integral distributions.

This last result was the basis for the whole paper. In lectures at the Nordic

Received by the editors November 24, 1976.

AMS (MOS) subject classifications (1970). Primary 46F10; Secondary 53C15, $58 \mathrm{G} 15$. 
Summer School in Grebbestad (1975), we gave an "analytic" description of the line bundle in which Hörmander's symbol takes its values. This description suggested the possibility that a general symbol construction existed; it was found a few months later.

There are some precursors to our definition of the symbol, due to Calderon [1] and kojasiewicz [8], [9]. A similar localization process is used by Hörmander [4] and Melin [10]. I would like to thank L. Boutet de Monvel, B. Fuglede, and G. Grubb for calling these references to my attention.

I would like to thank K. Andersson for the invitation to the Nordic Summer School and for his encouragement. I would also like to thank K. Sklower for his contribution to Chapter II, as well as his comments on the rest of the manuscript. Y. Colin de Verdière and L. Hörmander have made many valuable comments, especially pertaining to Chapter II.

The debt to [6], both in form and in content, should be evident to anyone who is familiar with that paper.

Finally, I would like to express thanks to the National Science Foundation for financial support (grant \#MPS74-23180) during the early stages of this research, and to N. H. Kuiper for the hospitality of the I.H.E.S., where this paper was written.

\section{ElementARY THEORY}

1.0. Introduction. We present here the definition and elementary properties of the symbol of a distribution. By "elementary", we mean that the analytic machinery of Fourier analysis and stationary phase is not used. Except for the calculation of examples, we do not even use integration by parts.

The chapter begins with the localization process (1.1) and enough of its properties (1.2) to justify the local definition of the symbol (1.3). After proving some further invariance properties (1.4), (1.5), we define the symbol for vector-bundle-valued distributions on a manifold (1.6).

1.1. Localization and order of distributions. We begin by summarizing some notation and basic facts from [11] concerning distributions on $\mathbf{R}^{n}$. A subset $A$ of $\mathscr{D}\left(\mathbf{R}^{n}\right)$, the compactly supported $C^{\infty}$ complex valued functions on $\mathbf{R}^{n}$, is called bounded if all the elements of $A$ are supported in a fixed compact subset $K \subseteq \mathbf{R}^{n}$, and if the partial derivatives of each order of the elements of $A$ are uniformly bounded. The distribution space $\mathscr{D}^{\prime}\left(\mathbf{R}^{n}\right)$ consists of those linear functionals $g: \mathcal{D}\left(\mathbf{R}^{n}\right) \rightarrow \mathbf{C}$ which are bounded on each bounded subset. We write $\langle g, u\rangle$ rather than $g(u)$ for the action of $g \in \mathscr{D}^{\prime}\left(\mathbf{R}^{n}\right)$. A subset $C \subseteq \mathscr{D}^{\prime}\left(\mathbf{R}^{n}\right)$ is called bounded if, for each bounded $A \subseteq \mathscr{D}\left(\mathbf{R}^{n}\right),\langle C, A\rangle=$ $\{\langle g, u\rangle \mid g \in C, u \in A\}$ is bounded in $C$. Some proofs in this paper may be simplified by the use of Theorem IX of Chapter III of [11], which states that a 
sufficient condition for boundedness of $C \subseteq \mathcal{D}^{\prime}\left(\mathbf{R}^{n}\right)$ is that $\langle C, u\rangle$ be bounded for each fixed $u \in \mathscr{D}\left(\mathbf{R}^{n}\right)$; however, we will not use this fact in any essential way.

We wish to localize a distribution $g \in \mathcal{D}^{\prime}\left(\mathbf{R}^{n}\right)$ at the origin and, simultaneously, at a "cotangent vector" $\xi \in \mathbf{R}^{n^{*}}$. To do this, we will choose a real valued function $\varphi \in C^{\infty}\left(\mathbf{R}^{n}\right)$ with $\varphi(0)=0$ and $d \varphi(0)=\xi$ and consider the asymptotic behavior as $\tau \uparrow \infty$ of the family $g_{\varphi}^{\tau}$ of distributions defined as follows.

Definition 1.1.1. Let $g \in \mathscr{D}^{\prime}\left(\mathbf{R}^{n}\right)$ and let $\varphi: \mathbf{R}^{n} \rightarrow \mathbf{R}$ be a $C^{\infty}$ function with $\varphi(0)=0$. We define the distribution $g_{\varphi}^{\tau}$ for $\tau \geqslant 1$ by

$$
\left\langle g_{\varphi}^{\tau}, u\right\rangle=\left\langle g, \tau^{n / 2} e^{-i \tau \varphi(x)} u(\sqrt{\tau} x)\right\rangle
$$

EXAMPLE 1.1.2. If $g$ is given by an ordinary (locally integrable) function $g(x)$ in the sense that

$$
\langle g, u\rangle=\int g(x) u(x) d x
$$

then we have

$$
\left\langle g_{\varphi}^{\tau}, u\right\rangle=\int g(x) \tau^{n / 2} e^{-i \tau \varphi(x)} u(\sqrt{\tau} x) d x=\int g\left(\frac{x}{\sqrt{\tau}}\right) e^{-i \tau \varphi(x / \sqrt{\tau})} u(x) d x .
$$

Thus, $g_{\varphi}^{\tau}$ is given by the locally integrable function

$$
g_{\varphi}^{\tau}(x)=\left(g e^{-i \tau \varphi}\right)(x / \sqrt{\tau}) .
$$

Even when $g$ is not an ordinary function, we will still use the symbolic notation (1.1.2) for $\langle g, u\rangle$, so (1.1.3) may be thought of as a general formula for $g_{\varphi}^{\tau}$. Note that the family $g_{\varphi}^{\tau}$ is obtained from $g$ by the successive action of two one-parameter groups: multiplication by the rapidly oscillating (as $\tau \uparrow \infty$ ) function $e^{-i \tau \varphi}$ and magnification by composition with the transformation $x \mapsto x / \sqrt{\tau}$. The choice of the square root in (1.1.1) turns out to be the best adapted to a general theory. For certain problems, it may be useful to replace $\sqrt{\tau}$ by $\tau^{\alpha}$ for some $\alpha$ other than $1 / 2$ (see, for example, [5]).

The idea of combining magnification with multiplication by rapidly oscillating functions goes back at least as far as [4] (see also [1], [10]), where it is used in the localization of subelliptic estimates for differential operators with variable coefficients. Even earlier, kojasiewicz [8], [9] studied the limiting behavior of distributions under magnification.

To measure the growth rate of $g_{\varphi}^{\tau}$, we make the following definition.

DeFINITION 1.1.3. If $\mathcal{E}$ is any vector space with a distinguished class of subsets called "bounded sets", we denote by $S^{N}(\mathcal{E})$ the set of families $[1, \infty) \ni \tau \mapsto g^{\tau} \in \mathcal{E}$ for which the set $\left\{\tau^{-N} g^{\tau} \mid \tau \geqslant 1\right\}$ is bounded. The intersection $\bigcap_{N \in \mathbf{R}} S^{N}(\mathcal{E})$ is denoted by $S^{-\infty}(\mathcal{E})$. If $\tau \mapsto g^{\tau}$ belongs to $S^{N}(\mathcal{E})$, we write $g^{\tau}=O\left(\tau^{N}\right)$. 
LEMMA 1.1.4. For any real $N$ and $\tau_{0} \geqslant 1$, the set $\left\{\tau^{N} g_{\varphi}^{\tau} \mid \tau_{0} \geqslant \tau \geqslant 1\right\}$ is bounded in $\mathscr{D}^{\prime}\left(\mathbf{R}^{n}\right)$.

Proof. Since $\left\langle\tau^{N} g_{\varphi}^{\tau}, u\right\rangle=\left\langle g, \tau^{N+n / 2} e^{-i \tau \varphi(x)} u(\sqrt{\tau} x)\right\rangle$ and $g$ is bounded on each bounded subset of $\mathscr{D}\left(\mathbf{R}^{n}\right)$, the conclusion of the lemma reduces to the fact that $\left\{\tau^{N+n / 2} e^{-i \Gamma \varphi(x)} u(\sqrt{\tau} x) \mid \tau_{0} \geqslant \tau \geqslant 1, u \in A\right\}$ is bounded for each bounded set $A$.

LEMMA 1.1.5. The truth of the statement " $g_{\varphi}^{\tau}=O\left(\tau^{N}\right)$ " depends only on the germs of $g$ and $\varphi$ at the origin.

Proof. Suppose that $g$ agrees with $h$ and $\varphi$ agrees with $\psi$ on some neighborhood $\mathcal{Q}$ of the origin, and that $g_{\varphi}^{\tau}=O\left(\tau^{N}\right)$. Let $A \subseteq \mathscr{D}\left(\mathbf{R}^{n}\right)$ be any bounded set of test functions, supported in the compact subset $K$. If $\tau_{0}$ is large enough so that $K \subseteq \tau_{0} \mathcal{Q}$, then $\left\langle g_{\varphi}^{\tau}, u\right\rangle=\left\langle h_{\psi}^{\tau}, u\right\rangle$ for all $u \in A$ and $\tau \geqslant \tau_{0}$. Since $g_{\varphi}^{\tau}=O\left(\tau^{N}\right)$, the set $\left\{\left\langle\tau^{-N} h_{\psi}^{\tau}, u\right\rangle \mid \tau \geqslant \tau_{0}, u \in A\right\}$ is bounded. By Lemma 1.1.4, the set $\left\{\left\langle\tau^{-N} h_{\psi}^{\tau}, u\right\rangle \mid \tau_{0} \geqslant \tau \geqslant 1, u \in A\right\}$ is bounded as well. Since the union of two bounded sets is bounded, $\left\{\left\langle\tau^{-N} h_{\psi}^{\tau}, u\right\rangle \mid \tau \geqslant 1, u \in A\right\}$ is bounded. $A$ was any bounded set, so we have $h_{\psi}^{\tau} \in O\left(\tau^{N}\right)$.

LEMMA 1.1.6. For every $g$ and $\varphi$, there exists an $N$ such that $g_{\varphi}^{\tau}=O\left(\tau^{N}\right)$.

Proof. Suppose first that $g$ is a measure. If $A$ is any bounded set of test functions, the set $\left\{e^{-i \pi \varphi(x)} u(\sqrt{\tau} x) \mid \tau \geqslant 1, u \in A\right\}$ is bounded in the uniform norm, so the set

$$
\left\{\left\langle\tau^{-n / 2} g_{\varphi}^{\tau}, u\right\rangle \mid \tau \geqslant 1, u \in A\right\}=\left\{\left\langle g, e^{-i \tau \varphi(x)} u(\sqrt{\tau} x)\right\rangle \mid \tau \geqslant 1, u \in A\right\}
$$

is bounded because $g$ is a measure, and we have $g_{\varphi}^{\tau}=O\left(\tau^{n / 2}\right)$.

For the general case, we may assume by Lemma 1.1.5 that $g$ has compact support. By the results of $\S I I I, 7$ of [11], $g$ has finite order $m$ and is therefore a finite sum of $m$ th derivatives of measures. Now if $A$ is a bounded set, the set of $m$ th derivatives of the elements of $\left\{\tau^{-m} e^{-i r \varphi(x)} u(\sqrt{\tau} x) \mid \tau \geqslant 1, u \in A\right\}$ is bounded in the uniform norm. Repeating the previous argument and using the fact that a finite sum of bounded sets is bounded leads to the conclusion that $g_{\varphi}^{\tau}=O\left(\tau^{m+n / 2}\right)$.

DEFINITION 1.1.7. If the set $S=\left\{N \mid g_{\varphi}^{\tau}=O\left(\tau^{N}\right)\right\}$ is of the form $[a, \infty)$, we define the order of $g$ at $(0, \varphi)$ to be $a$, and we denote it by $O_{(0, \varphi)}(g)$. If $S=(-\infty, \infty)$, we define $O_{(0, \varphi)}(g)=-\infty$. If $S=(a, \infty)$, we define $O_{(0, \varphi)}(g)$ to be $a^{+}$, where $a^{+}$is a "fictitious number" considered to be greater than $a$ but less than every number in $(a, \infty)$.

It follows from Lemma 1.1.5 and the proof of Lemma 1.1.4 that $O_{(0, \varphi)}(g)$ depends only upon the germs of $g$ and $\varphi$ at the origin. Furthermore, $O_{(0, p)}(g) \leqslant n / 2+m$, where $m$ is the distribution order of $\mathcal{X} g$ for any $\mathcal{X} \equiv 1$ near the origin, and $O_{(0,0)}(g) \leqslant n / 2+m / 2$. 
1.2. Change of phase function. Our first main result concerns the dependence of $g_{\varphi}^{\tau}$ (and hence $O_{(0, \varphi)}(g)$ ) upon the choice of $\varphi$.

Proposition 1.2.1. Let $\psi(x)=\varphi(x)+q(x)+r(x)$, where $q$ is a homogeneous quadratic polynomial and $r(x)$ vanishes to order at least 3 at the origin. Then $O_{(0, \varphi)}(g) \leqslant N$ implies

$$
g_{\psi}^{\tau}-e^{-i q} g_{\varphi}^{\tau}=O\left(\tau^{N-1 / 2}\right) .
$$

Proof. By the quadratic homogeneity of $q, \tau q(x / \sqrt{\tau})=q(x)$, so $g_{\varphi+q}^{\tau}$ is equal to $e^{-i q} g_{\varphi}^{\tau}$. We conclude that $g_{\varphi+q}^{\tau}=O\left(\tau^{N}\right)$; writing

$$
\begin{aligned}
g_{\varphi+q+r}^{\tau}-e^{-i q} g_{\varphi}^{\tau} & =g_{\varphi+q+r}^{\tau}-g_{\varphi+q}^{\tau}+g_{\varphi+q}^{\tau}-e^{-i q} g_{\varphi}^{\tau} \\
& =g_{\varphi+q+r}^{\tau}-g_{\varphi+q}^{\tau}
\end{aligned}
$$

and substituting $\varphi+q$ for $\varphi$, we have reduced the problem to the case $q=0$. Assuming, then, that $\psi=\varphi+r$, we have

$$
\begin{aligned}
\tau^{-n / 2}\left\langle g_{\psi}^{\tau}-g_{\varphi}^{\tau}, u\right\rangle & =\left\langle g,\left\{e^{-i \tau(\varphi(x)+r(x))}-e^{-i \tau \varphi(x)}\right\} u(\sqrt{\tau} x)\right\rangle \\
& =\left\langle g^{-i \tau \varphi(x)}\left\{e^{-i \tau r(x)}-1\right\} u(\sqrt{\tau} x)\right\rangle \\
& =\left\langle g, e^{-i \tau \varphi(x)} v_{u}^{\tau}(\sqrt{\tau} x)\right\rangle,
\end{aligned}
$$

where

$$
v_{u}^{\tau}(x)=\left[e^{-i \tau r(x / \sqrt{\tau})}-1\right] u(x) .
$$

It suffices to show that $B_{A}=\left\{\tau^{1 / 2} v_{u}^{\tau}(x) \mid \tau \geqslant 1, u \in A\right\}$ is bounded whenever $A$ is bounded, for we will have

$$
\tau^{-(N-1 / 2)}\left\langle g_{\psi}^{\tau}-g_{\varphi}^{\tau}, u\right\rangle=\tau^{-N+1 / 2}\left\langle g_{\varphi}^{\tau}, v_{u}^{\tau}\right\rangle=\tau^{-N}\left\langle g_{\varphi}^{\tau}, \tau^{1 / 2} v_{u}^{\tau}\right\rangle
$$

which, since $g_{\varphi}^{\tau}=O\left(\tau^{N}\right)$, will be bounded for $\tau \geqslant 1, u \in A$.

To show that $B_{A}$ is bounded, we begin by choosing a function $\mathscr{X} \in \mathscr{D}\left(\mathbf{R}^{n}\right)$ which is identically 1 on the supports of all $u$ in $A$. Then

$$
v_{u}^{\tau}(x)=\left[e^{-i \tau r(x / \sqrt{\tau})}-1\right] \mathfrak{X}(x) u(x) .
$$

Since the product of two bounded sets is bounded (by Leibniz' rule for derivatives), it suffices to show the boundedness of

$$
B_{\mathcal{X}}=\left\{\tau^{1 / 2}\left[e^{-i \pi r(x / \sqrt{\tau})}-1\right] \mathcal{X}(x) \mid \tau \geqslant 1\right\} .
$$

Since $r$ vanishes to order at least 3 at the origin, Taylor's formula gives

$$
r(x)=\sum_{j, k, l} r_{j k l}(x) x_{j} x_{k} x_{l}
$$

with $r_{j k l}$ smooth. Hence, the typical element of $B_{\mathscr{X}}$ may be written as

$$
w_{\tau}(x)=\tau^{1 / 2}\left[e^{-i / \sqrt{\tau} \sum r_{j k 1}(x / \sqrt{\tau}) x_{j} x_{k} x_{i}}-1\right] \mathcal{X}(x) .
$$


Writing $e^{-i y}-1=y h(y)$, where $h$ is $C^{\infty}$, we have

$$
\begin{aligned}
& w_{\tau}(x)=\tau^{1 / 2}\left[\tau^{-1 / 2} \sum r_{j k l}(x / \sqrt{\tau}) x_{j} x_{k} x_{l} h\right. \\
& \left.\cdot\left(1 / \sqrt{\tau} \sum r_{j k l}(x / \sqrt{\tau}) x_{j} x_{k} x_{l}\right)\right] \chi(x) \\
& =\sum r_{j k l}(x / \sqrt{\tau}) x_{j} x_{k} x_{l} h\left(1 / \sqrt{\tau} \sum r_{j k l}(x / \sqrt{\tau}) x_{j} x_{k} x_{l}\right) \chi(x) \text {. }
\end{aligned}
$$

Let $K$ be a closed ball about the origin containing the support of $\mathcal{X}$. By estimating the derivatives of $\mathcal{X}$ and $r_{j k l}$ on $K$, and the derivatives of $h$ on the compact $\operatorname{set}\left\{\sum r_{j k l}(y) x_{j} x_{k} x_{l} \mid x, y \in K\right\}$, we obtain estimates, uniform in $\tau$, for the derivatives of $w_{\tau}(x)$, so $B_{\odot x}$ is bounded.

COROllaRY 1.2.2. The order $O_{(0, \varphi)}(g)$ depends upon $\varphi$ only through its differential $d \varphi(0)$.

Proof. Suppose that $g_{\varphi}^{\tau}=O\left(\tau^{N}\right)$, and $d \varphi(0)=d \psi(0)$. Then $\psi(x)=\varphi(x)$ $+q(x)+r(x)$, as in Proposition 1.2.1, and we have $g_{\psi}^{\tau}-e^{-i q} g_{\varphi}^{\tau}=$ $O\left(\tau^{N-1 / 2}\right)$, so

$$
g_{\psi}^{\tau}=e^{-i q} g_{\varphi}^{\tau}+O\left(\tau^{N-1 / 2}\right)=e^{-i q} O\left(\tau^{N}\right)+O\left(\tau^{N-1 / 2}\right)=O\left(\tau^{N}\right) .
$$

Definition 1.2.3. For $g \in \mathscr{D}^{\prime}\left(\mathbf{R}^{n}\right)$ and $\xi \in \mathbf{R}^{n^{*}}$, we define the order $O_{(0, \xi)}(g)$ of $g$ at $(0, \xi)$ to be $O_{(0, \varphi)}(g)$, for any $\varphi$ such that $d \varphi(0)=\xi$.

In computing $O_{(0, \xi)}(g)$, it is often convenient to take $\varphi$ to be the linear function $\varphi(x)=\langle\xi, x\rangle$; in this case, we will write $g_{\xi}^{\tau}$ for $g_{\varphi}^{\tau}$.

EXAMPLE 1.2.4. Let $g$ be a $C^{k}$ function vanishing to order at least $k$ at 0 , i.e. $g(x)=\sum_{|\alpha|=k} c_{\alpha}(x) x^{\alpha}$, where the $c_{\alpha}$ 's are continuous functions. We compute $O_{(0,0)}(g)$ by looking at

$$
g_{0}^{\tau}(x)=\tau g\left(\frac{x}{\sqrt{\tau}}\right)=\tau^{-k / 2} \sum_{|\alpha|=k} c_{\alpha}\left(\frac{x}{\sqrt{\tau}}\right) x^{\alpha} .
$$

The continuous functions $\tau^{k / 2} g_{0}^{\tau}(x)=\sum_{|\alpha|=k} c_{\alpha}(x / \sqrt{\tau}) x^{\alpha}$ are uniformly bounded on compact sets, so they are bounded in $\mathscr{D}^{\prime}\left(\mathbf{R}^{n}\right)$, and we conclude that $O_{(0,0)}(g) \leqslant-k / 2$. If $c_{\alpha}(0) \neq 0$ for some $\alpha$, i.e. if $g$ vanishes exactly to order $k$, we can conclude that $O_{(0,0)}(g)=-k / 2$. (Use a test function $u$ supported in a set on which $\Sigma_{|\alpha|=k} c_{\alpha}(0) x^{\alpha}>0$ )

For $\xi \neq 0$, we have

$$
\left\langle g_{\xi}^{\tau}, u\right\rangle=\int g\left(\frac{x}{\sqrt{\tau}}\right) e^{-i \tau\langle\xi, x / \sqrt{\tau}\rangle} u(x) d x=\int e^{-i \sqrt{\tau}\langle\xi, x\rangle} g\left(\frac{x}{\sqrt{\tau}}\right) u(x) d x .
$$

Since $\xi \neq 0$, we may integrate by parts $k$ times with the operator $L=$ $\Sigma \xi_{i} \partial / \partial x_{i}$ to obtain

$$
\left\langle g_{\xi}^{\tau}, u\right\rangle=\left(\frac{-1}{-i \sqrt{\tau}|\xi|^{2}}\right)^{k} \int e^{-i \sqrt{\tau}\langle\xi, x\rangle} L^{k}\left(g\left(\frac{x}{\sqrt{\tau}}\right) u(x)\right) d x .
$$


Now

$$
L^{k}\left(g\left(\frac{x}{\sqrt{\tau}}\right) u(x)\right)=\sum_{j=0}^{k}\left(\begin{array}{l}
k \\
j
\end{array}\right) \tau^{-j / 2}\left(L^{j} g\right)\left(\frac{x}{\sqrt{\tau}}\right) \cdot\left(L^{k-j} u\right)(x) .
$$

Since $g$ vanishes to order at least $k$ at the origin, $L^{j} g$ vanishes to order at least $k-j$ there, and the estimate

$$
\left(L^{j} g\right)(x / \sqrt{\tau})=O\left(\tau^{-(k-j) / 2}\right)
$$

holds uniformly on bounded sets of $x$. It follows from (1.2.2) that, for any bounded set $A$ in $\mathscr{D}\left(\mathbf{R}^{n}\right)$, the functions $\left\{\tau^{k / 2} L^{k}(g(x / \sqrt{\tau}) u(x)) \mid \tau \geqslant 1, u \in\right.$ $A\}$ are uniformly bounded on $\mathbf{R}^{n}$. Since the supports of these functions are contained in a fixed bounded set, it follows from (1.2.1) that $g_{\xi}^{\tau}=$ $O\left(\tau^{-k / 2-k / 2)}=O\left(\tau^{-k}\right)\right.$, and $O_{(0, \xi)}(g) \leqslant-k$.

If we ignore the vanishing of $g$ at the origin, we still have uniform boundedness for $\left\{L^{k} g(x / \sqrt{\tau}) u(x) \mid \tau \geqslant 1, u \in A\right\}$, and we conclude that $O_{(0, \xi)}(g) \leqslant-k / 2$. In particular, if $g$ is $C^{\infty}$ near the origin, we have $O_{(0, \xi)}=$ $-\infty$. Now if $g$ is $C^{k}$ near the origin, it is the sum of a polynomial and a function which vanishes to order $k$, so we may conclude that $O_{(0, \xi)} \leqslant-k$. To summarize:

Proposition 1.2.5. Let $g$ be equal to a $C^{l}$ function on a neighborhood of the origin. Then $O_{(0, \xi)}(g)=-l$ for $\xi \neq 0$, while $O_{(0,0)}(g)=-k / 2$, where $k$ is the order of vanishing of $g$ at the origin.

EXAMPLE 1.2.6. Let $g(x)=\delta\left(x_{1}\right) \delta\left(x_{2}\right) \cdots \delta\left(x_{r}\right)$ for some $r \leqslant n$, i.e.

$$
\langle g, u\rangle=\int u\left(0,0, \ldots, 0, x_{r+1}, \ldots, x_{n}\right) d x_{r+1} \cdots d x_{n} .
$$

Then we have

$$
\begin{aligned}
& \left\langle g_{\xi}^{\tau}, u\right\rangle \\
& \quad=\tau^{n / 2} \int e^{-i \tau \sum_{j-r+1}^{n} \xi x_{j}} u\left(0,0, \ldots, 0, \sqrt{\tau} x_{r+1}, \ldots, \sqrt{\tau} x_{n}\right) d x_{r+1} \cdots d x_{n} \\
& \quad=\tau^{r / 2} \int e^{-i V_{\tau} \sum_{j-r+1}^{n} \xi, x_{j}} u\left(0,0, \ldots, 0, x_{r+1}, \ldots, x_{n}\right) d x_{r+1} \cdots d x_{n} .
\end{aligned}
$$

If $\xi_{r+1}=\cdots=\xi_{n}=0$, we have $O_{(0, \xi)}(g)=r / 2$, while if $\xi_{r+1}$ through $\xi_{n}$ are not all zero we can integrate by parts as in Example 1.2.4 to conclude that $O_{(0, \xi)}(g)=-\infty$.

The order $O_{(0, \xi)}(g)$ appears to be a measure of the degree of singularity of the distribution $g$ at 0 , as observed in the direction $\xi$. We will show in $\$ 2.2$ that $O_{(0, \xi)}(g)=-\infty$ if $\xi \neq 0$ and $(0, \xi)$ does not belong to the wavefront set (see [6]) of $g$. 
1.3. Definition of the symbol. If $O_{(0, \xi)}(g)<N$, we will define the symbol of order $N$ of $g$ at $(0, \xi)$ to be, essentially, the image of $g_{\varphi}^{\tau}$ in $S^{N}\left(\mathscr{D}^{\prime}\left(\mathbf{R}^{n}\right)\right) / S^{N-1 / 2}\left(\mathscr{D}^{\prime}\left(\mathbf{R}^{n}\right)\right)$, where $d \varphi(0)=\xi$. The following definitions are designed to accommodate the dependence of $g_{\varphi}^{\tau}$ on the quadratic part of $\varphi$.

Definition 1.3.1. For $\xi \in \mathbf{R}^{n^{*}}$, we define $g_{(0, \xi)}$ to be the set of equivalence classes $[\varphi]$ of real valued $C^{\infty}$ functions $\varphi$ such that $\varphi(0)=0, d \varphi(0)=\xi$, two functions $\varphi_{1}$ and $\varphi_{2}$ being considered equivalent if $\varphi_{1}-\varphi_{2}$ vanishes to order at least three at 0 . The equivalence class $[\varphi]$ is called the 2-jet of $\varphi$.

REMARKs 1.3.2. (a) If $[\varphi]$ and $[\psi]$ are any two elements of $g_{(0, \xi)}$, there is a unique homogeneous quadratic polynomial $q$ on $\mathbf{R}^{n}$ such that $[\psi]=[\varphi+q]$. Thus, $g_{(0, \xi)}$ is an affine space modeled on the vector space of quadratic functions.

(b) We can identify the elements of $g_{(0, \xi)}$ with certain lagrangian subspaces (see [ஞ]) of the tangent space at $(0, \xi)$ to the cotangent bundle $T^{*} \mathbf{R}^{n} \approx \mathbf{R}^{n} \times$ $\mathbf{R}^{n^{*}}$. In fact, given $\varphi$ with $d \varphi(0)=\xi$, the graph $\left\{(x, d \varphi(x)) \mid x \in \mathbf{R}^{n}\right\}$ of the differential $d \varphi$ is a lagrangian submanifold of $T^{*} R^{n}$, passing through $(0, \xi)$, whose tangent space at $(0, \xi)$ depends only upon the 2-jet $[\varphi]$. Every lagrangian subspace of $T_{(0, \xi)} T^{*} R^{n}$ which is transversal to the "vertical" $T_{(0, \xi)} T_{0}^{*} R^{n}$ arises in this way from an element of $g_{(0, \xi)}$.

Definition 1.3.3. Let $\delta$ be any module over $C^{\infty}\left(\mathbf{R}^{n}\right)$. For any $\xi \in \mathbf{R}^{n^{*}}$, we define the "twisted" space $\tilde{\varepsilon}_{(0, \xi)}$ to be the set of mappings $[\varphi] \mapsto g_{[\varphi]}$ from $g_{(0, \xi)}$ to $\mathcal{E}$ such that

$$
g[\varphi+q]=e^{-i q} g_{[\varphi]}
$$

for each quadratic function $q$.

It follows from Remarks 1.3.2 that, for any $[\varphi] \in \mathscr{g}_{(0, \xi)}$, the evaluation map $g_{[]} \mapsto g_{[\varphi]}$ is an isomorphism from $\tilde{E}_{(0, \xi)}$ to $\mathcal{E}$. Any structure on $\mathcal{E}$ which is left invariant by all operators of multiplication by $e^{-i q}, q$ quadratic, may be carried over to a structure of the same type on $\tilde{E}_{(0, \xi)}$. For example, $\tilde{E}_{(0, \xi)}$ is always a module over $C^{\infty}\left(\mathbf{R}^{n}\right)$. Furthermore, if $\mathcal{E}$ has an invariant class of bounded sets, we can define the spaces $S^{N}\left(\tilde{\delta}_{(0, \xi)}\right)$.

Definition 1.3.4. For $\xi \in \mathbf{R}^{n^{*}}$, we define the symbol space $\delta_{(0, \xi)}^{N}\left(\mathbf{R}^{n}\right)$ to be

$$
\left.\widehat{\left[S^{N}\left(\mathscr{D}^{\prime}\left(\mathbf{R}^{n}\right)\right) / S^{N-1 / 2}\left(\mathscr{Q}^{\prime}\left(\mathbf{R}^{n}\right)\right)\right.}\right]_{(0, \xi)} \text {. }
$$

REMARK 1.3.5. $\delta_{(0, \xi)}^{N}\left(\mathbf{R}^{n}\right)$ is naturally isomorphic to

$$
\left.\left.S^{N}\left(\widetilde{\mathcal{D}^{\prime}\left(\mathbf{R}^{n}\right.}\right)_{(0, \xi)}\right) / S^{N-1 / 2}\left(\widetilde{\mathbb{D}^{\prime}\left(\mathbf{R}^{n}\right.}\right)_{(0, \xi)}\right) \text {. }
$$

Definition 1.3.6. Given $g \in \mathscr{D}^{\prime}\left(\mathbf{R}^{n}\right)$ and $\xi \in \mathbf{R}^{n^{*}}$ such that $O_{(0, \xi)}(g)<N$, we define the symbol of order $N \sigma_{(0, \xi)}^{N}(g)$ of $g$ at $(0, \xi)$ to be the element of $\delta_{(0, \xi)}^{N}\left(\mathbf{R}^{n}\right)$ which assigns to each $[\varphi] \in \mathscr{g}_{(0, \xi)}$ the image in $S^{N}\left(\mathscr{D}^{\prime}\left(\mathbf{R}^{n}\right)\right) / S^{N-1 / 2}\left(\mathcal{D}^{\prime}\left(\mathbf{R}^{n}\right)\right)$ of the family $g_{\varphi}^{\tau}$ of Definition 1.1.1. 
It is Proposition 1.2.1 which makes this definition allowable. In computing $\sigma_{(0, \xi)}^{N}(g)$ for a specific $g$, it suffices to determine the behavior modulo $S^{N-1 / 2}\left(\mathscr{D}^{\prime}\left(\mathbf{R}^{n}\right)\right)$ of the family $g_{\varphi}^{\tau}$ for a particular $\varphi$ with $d \varphi(0)=\xi$. In practice, we usually use $\varphi(x)=\langle\xi, x\rangle$, perhaps after a change of coordinates adapted to the distribution $g$ under study.

The next lemma is a trivial consequence of Definition 1.3.6.

LEMMA 1.3.7. (a) If $O_{(0, \xi)}(g) \leqslant N$, then $O_{(0, \xi)}(g) \leqslant N-1 / 2$ if and only if $\sigma_{(0, \xi)}^{N}(g)=0$.

(b) If $O_{(0, \xi)}\left(g_{1}\right) \leqslant N$ and $O_{(0, \xi)}\left(g_{2}\right) \leqslant N$, then

$$
\sigma_{(0, \xi)}^{N}\left(g_{1}+g_{2}\right)=\sigma_{(0, \xi)}^{N}\left(g_{1}\right)+\sigma_{(0, \xi)}^{N}\left(g_{2}\right) \text {. }
$$

EXAMPLE 1.3.8. Let $g$ be a $C^{k+1}$ function vanishing to order at least $k$ at 0 . We may write $g(x)=\sum_{|\alpha|=k} c_{\alpha} x^{\alpha}+h(x)$, where the $c_{\alpha}$ 's are constants and $h$ is a $C^{k+1}$ function vanishing to order at least $k+1$ at 0 .

For $\xi=0$, we found in Example 1.2.4 that $O_{(0,0)}(g) \leqslant-k / 2$, so it is natural to compute the symbol of order $-k / 2$. But $O_{(0,0)}(h)<-k / 2-$ $1 / 2$, so by Lemma 1.3 .7 we have

$$
g_{0}^{\tau}(x) \equiv \tau^{-k / 2} \sum_{|\alpha|=k} c_{\alpha} x^{\alpha} \bmod S^{-k / 2-1 / 2}\left(\mathcal{D}^{\prime}\left(\mathbf{R}^{n}\right)\right) .
$$

Thus, the symbol $\sigma_{(0,0)}^{-k / 2}(g)$ is essentially $\tau^{-k / 2}$ times the terms of degree $k$ in the Taylor series for $g$ at the origin.

For $\xi \neq 0$, we have by Example 1.2.4 that $O_{(0, \xi)}(g) \leqslant-k$, so the natural symbol to calculate is that of order $-k$; but $O_{(0, \xi)}(h) \leqslant-k-1$ and

so $\sigma_{(0, \xi)}^{-k}(g)=0$.

$$
O_{(0, \xi)}\left(\sum_{|\alpha|=k} c_{\alpha} x^{\alpha}\right)=-\infty \quad \text { (Proposition 1.2.5), }
$$

EXAMPLE 1.3.9. Let $g(x)=\delta\left(x_{1}\right) \cdots \delta\left(x_{r}\right)$, as in Example 1.2.6. We saw there that $O_{(0, \xi)}(g)=r / 2$ if $\xi_{r+1}=\cdots=\xi_{n}=0$ and $O_{(0, \xi)}(g)=-\infty$ otherwise. When $\xi_{r+1}=\cdots=\xi_{n}=0$, we have

$$
\left\langle g_{\xi}^{\tau}, u\right\rangle=\tau^{r / 2} u\left(0, \ldots, 0, x_{r+1}, \ldots, x_{n}\right) d x_{r+1} \cdots d x_{n}=\tau^{r / 2}\langle g, u\rangle,
$$

so the symbol $\sigma_{(0, \xi)}^{r / 2}(g)$ is essentially $\tau^{r / 2}$ times $g$ itself.

EXAMPLE 1.3.10. Let $n=1, g(x)=\delta^{(m)}(x), m \geqslant 0$; i.e. $\langle g, u\rangle=$ $(-1)^{m} u^{(m)}(0)$. Then

$$
\begin{aligned}
\left\langle g_{\xi}^{\tau}, u\right\rangle & =\tau^{1 / 2}\left\langle g, e^{-i \tau \xi x} u(\sqrt{\tau} x)\right\rangle=\tau^{1 / 2}(-1)^{m}\left\{e^{-i \tau \xi x} u(\sqrt{\tau} x)\right\}^{(m)}(0) \\
& =\tau^{1 / 2}\left\{(i \tau \xi)^{m} u(0)+O\left(\tau^{m-1 / 2}\right)\right\} .
\end{aligned}
$$

If $\xi \neq 0$, we have $O_{(0, \xi)}\left(\delta^{(m)}\right)=m+1 / 2$, and $\left\{\delta^{(m)}\right\}_{\xi}^{\tau} \equiv \tau^{m+1 / 2}(i \xi)^{m} \delta^{(0)}$ $\bmod O\left(\tau^{m}\right)$, so the symbol of order $m+1 / 2$ of $\delta^{(m)}$ is essentially $\tau^{m+1 / 2}$ times $(i \xi)^{m} \delta^{(0)}$. 
If $\xi=0$, we have $\left\langle\left\{\delta^{(m)}\right\}_{0}^{\tau}, u\right\rangle=\tau^{1 / 2}(-1)^{m}\{u(\sqrt{\tau} x)\}^{(m)}(0)=$ $\tau^{m / 2+1 / 2}(-1)^{m} u^{(m)}(0)$, so $\left\{\delta^{(m)}\right\}_{0}^{\tau}=\tau^{m / 2+1 / 2} \delta^{(m)}, O_{(0,0)}\left(\delta^{(m)}\right)=m / 2+1 / 2$, and the symbol of $\delta^{(m)}$ at $(0,0)$ is essentially $m / 2+1 / 2$ times $\delta^{(m)}$ itself.

We leave it to the reader to verify that everything we have stated remains true if $m \leqslant 0$, e.g. for the Heaviside function

$$
\delta^{(-1)}(x)=\left\{\begin{array}{ll}
0 & x \leqslant 0 \\
1 & x>0
\end{array}\right\}
$$

In all the examples above, the symbol exhibited a certain homogeneity with respect to $\tau$. We will now formalize this situation with some definitions.

DEFINITION 1.3.11. For each real number $N$, we define an embedding $\left.c_{N}: \widetilde{D^{\prime}\left(\mathbf{R}^{n}\right.}\right)_{(0, \xi)} \rightarrow \mathcal{S}_{(0, \xi)}^{N}\left(\mathbf{R}^{n}\right)$ by assigning to each element $h$ of $\widetilde{\mathcal{D}^{\prime}\left(\mathbf{R}^{n}\right)_{(0, \xi)}}$ the image in

$$
\left.\left.S^{N}\left(\widetilde{\mathcal{D}^{\prime}\left(\mathbf{R}^{n}\right.}\right)_{(0, \xi)}\right) / S^{N-1 / 2}\left(\widetilde{\mathcal{D}^{\prime}\left(\mathbf{R}^{n}\right.}\right)_{(0, \xi)}\right)
$$

of the family $\tau \mapsto \tau^{N} h$.

Definition 1.3.12. Let $O_{(0, \xi)}(g) \leqslant N$, and suppose that $\sigma_{(0, \xi)}^{N}(g)$ belongs to the image of $C_{N}$. Then we say that $g$ is homogeneous of degree $N$ at $(0, \xi)$ and we call $\left.C_{N}^{-1}\left(\sigma_{(x, \xi)}^{N}(g)\right) \in \widetilde{\mathbb{D}^{\prime}\left(\mathbf{R}^{n}\right.}\right)_{(0, \xi)}$ the homogeneous $N$-symbol of $g$ at $(0, \xi)$.

To establish that a given distribution $g$ is homogeneous of degree $N$ at $(0, \xi)$, it suffices to check that, for a particular $\varphi$ with $d \varphi(0)=\xi, g_{\varphi}^{\tau}=\tau_{\varphi}^{N} \gamma+$ $O\left(\tau^{N-1 / 2}\right)$, where $\gamma$ is a fixed element of $\mathscr{D}^{\prime}\left(\mathbf{R}^{n}\right)$. The element of $\mathcal{D}^{\prime}\left(\mathbf{R}^{n}\right)_{(0, \xi)}$ which assigns $\gamma$ to $[\varphi]$ is then the homogeneous $N$-symbol of $g$ at $(0, \xi)$. For instance, the computation of Example 1.3.10 shows that $\delta^{(m)}$ is homogeneous of order $m+1 / 2$ [order $m / 2+1 / 2]$ at $(0, \xi)$ if $\xi \neq 0[\xi=0]$, and its principal symbol is $\delta^{(0)}\left[\delta^{(m)}\right]$.

Here is an example of a nonhomogeneous distribution.

EXAMPLE 1.3.13. Let $g(x)=1 /\left(x^{2}-\ln \left(x^{2}\right)\right)(g(0)=0)$. For $\xi=0$, we have

$$
g_{0}^{\tau}(x)=g\left(\frac{x}{\sqrt{\tau}}\right)=\frac{\tau}{x^{2}-\tau \ln x^{2}+\tau \ln \tau} .
$$

As $\tau \rightarrow \infty, g_{0}^{\tau}(x)$ approaches zero uniformly on compact sets, but more slowly than any power of $\tau$. By testing $g_{0}^{\tau}$ against nonnegative functions, we find that $O_{(0,0)}(g)=0$, but $g$ is clearly not homogeneous at $(0,0)$. (Note that, by Example 1.2.4, 0 is the largest order which a continuous function can have. Any continuous function vanishing at 0 and satisfying a Hölder condition of order $\alpha$ at 0 would have order $\leqslant-\alpha / 2$ at $(0,0)$.)

To give a description of the symbol, and also to analyze the case $\xi \neq 0$, we write

$$
g\left(\frac{x}{\sqrt{\tau}}\right)=\frac{1}{\ln \tau}\left\{1 / 1-\frac{\ln x^{2}-x^{2} / \tau}{\ln \tau}\right\}
$$


Expanding this by the formula $1 /(1-a)=1+a+a^{2} /(1-a)$, we get

$$
\begin{aligned}
g\left(\frac{x}{\sqrt{\tau}}\right)= & \frac{1}{\ln \tau}\left(1+\frac{1}{\ln \tau}\left(\ln x^{2}-\frac{x^{2}}{\tau}\right)\right) \\
& +\frac{1}{(\ln \tau)^{2}}\left(\ln x^{2}-\frac{x^{2}}{\tau}\right)^{2} g\left(\frac{x}{\sqrt{\tau}}\right) .
\end{aligned}
$$

lt follows, first of all, that $g_{0}^{\tau}=g(x / \sqrt{\tau})=1 / \ln \tau+O\left(1 /(\ln \tau)^{2}\right)$, so we have found the symbol of $g^{\tau}$ at $(0,0)$, modulo $1 /(\ln \tau)^{2}$.

For $\xi \neq 0$, we look at

$$
\begin{aligned}
& g_{\xi}^{\tau}= e^{-i \sqrt{\tau} \xi x} g(x / \sqrt{\tau}) \\
&=e^{-i \sqrt{\tau} \xi x}\left(\frac{1}{\ln \tau}+\frac{1}{(\ln \tau)^{2}}\left(\ln x^{2}-\frac{x^{2}}{\tau}\right)\right. \\
&\left.\quad+\frac{1}{(\ln \tau)^{2}}\left(\ln x^{2}-\frac{x^{2}}{\tau}\right)^{2} g\left(\frac{x}{\sqrt{\tau}}\right)\right] .
\end{aligned}
$$

The first term is $O\left(\tau^{-\infty}\right)$ since $\xi \neq 0$. The last term is $O\left(1 /(\ln \tau)^{3}\right)$, since $g(x / \sqrt{\tau})=O(1 / \ln \tau)$. We conclude that $g_{\xi}^{\tau}=O\left(1 /(\ln \tau)^{2}\right)$ and that

$$
g_{\xi}^{\tau} \equiv e^{-i V_{\tau} \xi x} \frac{\ln x^{2}}{(\ln \tau)^{2}} \quad \bmod O\left(\frac{1}{(\ln \tau)^{3}}\right) .
$$

Thus $g_{\xi}^{\tau}$ goes to zero at least "twice as fast" for $\xi \neq 0$ as for $\xi=0$. (We have not been able to determine the asymptotic behavior of

$$
\left.e^{-i \sqrt{\tau} \xi x} \ln x^{2} /(\ln \tau)^{2} .\right)
$$

1.4. Composition with diffeomorphisms. If $\theta: \mathbf{R}^{n} \rightarrow \mathbf{R}^{n}$ is a diffeomorphism and $g$ is an ordinary function on $\mathbf{R}^{n}$, we have the change of variables formula

$$
\int g(\theta(x)) u(x) d x=\int g(x) u\left(\theta^{-1}(x)\right) J_{\theta},(x) d x,
$$

where $J_{\theta}$, is the absolute value of the Jacobian determinant $\operatorname{det}\left(\partial\left(x_{i} \circ \theta^{-1}\right) / \partial x_{j}\right)$. If $g$ is any distribution, the pullback $g \circ \theta$ is accordingly defined by

$$
\langle g \circ \theta, u\rangle=\left\langle g, J_{\theta-1} \cdot\left(u \circ \theta^{-1}\right)\right\rangle .
$$

The following proposition shows that our symbols behave nicely under pullback.

Proposition 1.4.1. Let $\theta: \mathbf{R}^{n} \rightarrow \mathbf{R}^{n}$ be a diffeomorphism such that $\theta(0)=0$. Then $O_{(0, \varphi)}(g) \leqslant N$ implies

$$
(g \circ \theta)_{\varphi \circ \theta}^{\tau}-g_{\theta}^{\tau} \circ T_{0} \theta=O\left(\tau^{N-1 / 2}\right),
$$


where $T_{0} \theta: \mathbf{R}^{n} \rightarrow \mathbf{R}^{n}$ is the derivative of $\theta$ at 0 .

Proof. If $\theta$ is a linear mapping, it is easy to check that $(g \circ \theta)_{\varphi \circ \theta}^{\tau}$ is equal to $g_{\varphi}^{\tau} \circ T_{0} \theta$. On the other hand, the set of $\theta$ for which the proposition is true is closed under composition, so it suffices to consider the case where $T_{0} \theta$ is the identity mapping.

We have

$$
\begin{aligned}
\tau^{-n / 2} & \left\langle(g \circ \theta)_{\varphi \circ \theta}^{\tau}-g_{\varphi}^{\tau}, u\right\rangle \\
& =\int(g \circ \theta)(x) e^{-i \tau(\varphi \circ \theta)(x)} u(\sqrt{\tau} x) d x-\int g(x) e^{-i \tau \varphi(x)} u(\sqrt{\tau} x) d x \\
& =\int g(x) e^{-i \tau \varphi(x)} u\left(\sqrt{\tau} \theta^{-1}(x)\right) J_{\theta^{-1}}(x) d x-\int g(x) e^{-i \tau \varphi(x)} u(\sqrt{\tau} x) d x \\
& =\int g(x) e^{-i \tau \varphi(x)}\left\{u\left(\sqrt{\tau} \theta^{-1}(x)\right) J_{\theta^{-1}}(x)-u(\sqrt{\tau} x)\right\} d x .
\end{aligned}
$$

The proof now follows the pattern established in Proposition 1.2.1. The expression in braces in the last integral is equal to $v_{u}^{\tau}(\sqrt{\tau} x)$, where

$$
v_{u}^{\tau}(x)=u\left(\sqrt{\tau} \theta^{-1}(x / \sqrt{\tau})\right) J_{\theta^{-1}}(x / \sqrt{\tau})-u(x) .
$$

It suffices to show that the set $B_{A}=\left\{\tau^{1 / 2} v_{u}^{\tau}(x) \mid \tau \geqslant 1, u \in A\right\}$ is bounded whenever $A$ is bounded.

We will split up $v_{u}^{\tau}(x)$ as

$$
\begin{aligned}
v_{u}^{\tau}(x)= & {\left[u\left(\sqrt{\tau} \theta^{-1}(x / \sqrt{\tau})\right)-u(x)\right] J_{\theta^{-1}}(x / \sqrt{\tau}) } \\
& +u(x)\left[J_{\theta^{-1}}(x / \sqrt{\tau})-1\right]
\end{aligned}
$$

and analyze each of the bracketed expressions by Taylor's theorem. In fact, we have:

$$
u(y)=u(x)+\sum_{j=1}^{n} a_{j}^{u}(x, y)\left(y_{j}-x_{j}\right)
$$

where

$$
a_{j}^{u}(x, y)=\int_{0}^{1} \frac{\partial u}{\partial x_{j}}(x+t(y-x)) d t
$$

Also,

$$
\left[\theta^{-1}(x)\right]_{j}=x_{j}+\sum_{k, l=1}^{n} b_{j k l}(x) x_{k} x_{l},
$$

and

$$
J_{\theta^{-1}}(x)=1+\sum_{j=1}^{n} c_{j}(x) x_{j}
$$


From (1.4.5), we have

$$
\begin{aligned}
{\left[\sqrt{\tau} \theta^{-1}\left(\frac{x}{\sqrt{\tau}}\right)\right]_{j} } & =\sqrt{\tau}\left\{\frac{x_{j}}{\sqrt{\tau}}+\sum_{k, l=1}^{n} b_{j k l}\left(\frac{x}{\sqrt{\tau}}\right) \frac{x_{j} x_{k}}{\tau}\right\} \\
& =x_{j}+\tau^{-1 / 2} \sum_{j, k=1}^{n} b_{j k l}\left(\frac{x}{\sqrt{\tau}}\right) x_{j} x_{k} .
\end{aligned}
$$

Combining this with (1.4.3) gives

$$
\begin{aligned}
u(\sqrt{\tau} & \left.\theta^{-1}\left(\frac{x}{\sqrt{\tau}}\right)\right)-u(x) \\
& =\sum_{j=1}^{n} a_{j}^{u}\left(x, \sqrt{\tau} \theta^{-1}\left(\frac{x}{\sqrt{\tau}}\right)\right) \tau^{-1 / 2} \sum_{k, l=1}^{n} b_{j k l}\left(\frac{x}{\sqrt{\tau}}\right) x_{k} x_{l} \\
& =\tau^{-1 / 2} \sum_{j, k, l=1}^{n} a_{j}^{u}\left(x, \sqrt{\tau} \theta^{-1}\left(\frac{x}{\sqrt{\tau}}\right)\right) b_{j k l}\left(\frac{x}{\sqrt{\tau}}\right) x_{j} x_{k} .
\end{aligned}
$$

On the other hand, from (1.4.6) we have

$$
J_{\theta^{-1}}\left(\frac{x}{\sqrt{\tau}}\right)-1=\tau^{-1 / 2} \sum_{j=1}^{n} c_{j}\left(\frac{x}{\sqrt{\tau}}\right) x_{j}
$$

Substituting (1.4.7) and (1.4.8) into (1.4.2) gives

$$
\begin{aligned}
& \tau^{1 / 2} v_{u}^{\tau}(x) \\
& =\left[\sum_{j, k, l=1}^{n} a_{j}^{u}\left(x, \sqrt{\tau} \theta^{-1}\left(\frac{x}{\sqrt{\tau}}\right)\right) b_{j k l}\left(\frac{x}{\sqrt{\tau}}\right) x_{k} x_{l}\right] J_{\theta-1}\left(\frac{x}{\sqrt{\tau}}\right) \\
& \quad+u(x) \sum_{j=1}^{n} c_{j}\left(\frac{x}{\sqrt{\tau}}\right) x_{j}
\end{aligned}
$$

To show that the right-hand side of (1.4.9) is bounded, we choose a ball $K \subseteq \mathbf{R}^{n}$, centered at the origin, containing the supports of all $u \in A$. Since the bracketed expression in (1.4.9) is just $u\left(\sqrt{\tau} \theta^{-1}(x / \sqrt{\tau})\right)-u(x)$ it is zero unless $x$ or $\sqrt{\tau} \theta^{-1}(x / \sqrt{\tau})$ belongs to $K$, i.e. unless $x$ belongs to $K \cup \sqrt{\tau} \theta(1 / \sqrt{\tau} K)$. But this set is uniformly bounded for $\tau \geqslant 1$, since $\theta$ satisfies a uniform Lipschitz condition on $K$. Let $L$ be a compact set containing $K$ and all the $\sqrt{\tau} \theta(1 / \sqrt{\tau} K)$ for $\tau \geqslant 1$; then the bracketed expression in (1.4.9) vanishes unless $x$ (and hence $\sqrt{\tau} \theta^{-1}(x / \sqrt{\tau})$ ) belongs to $L$, and it follows from (1.4.9) that the support of $\tau^{1 / 2} v_{u}^{\tau}(x)$ is contained in $L$.

The only expression on the right-hand side of (1.4.9) whose derivatives are not uniformly bounded on $L$ is $a_{j}^{u}\left(x, \sqrt{\tau} \theta^{-1}(x / \sqrt{\tau})\right)$. But the integral representation (1.4.4) shows that the derivatives of $a_{j}^{n}(x, y)$ are uniformly (for $u \in A$ ) bounded on $L \times L$, and it is simple to check that the derivatives of 
$\sqrt{\tau} \theta^{-1}(x / \sqrt{\tau})$ are uniformly (for $\tau \geqslant 1$ ) bounded on $L$. Applying the chain rule, we are done.

If $\theta$ is a diffeomorphism with $\theta(0)=0$, and $\xi \in \mathbf{R}^{n^{*}}$, then $\xi \circ T_{0} \theta$ is also an element of $\mathbf{R}^{n^{*}}$. Combining the proposition just proven with Corollary 1.2.2, we have the following invariance property of the order.

Corollary 1.4.2. Let $\theta: \mathbf{R}^{n} \rightarrow \mathbf{R}^{n}$ be a diffeomorphism with $\theta(0)=0, g \in$ O $\mathcal{D}^{\prime}\left(\mathbf{R}^{n}\right)$, and $\xi \in \mathbf{R}^{n^{*}}$. Then $O_{\left(0, \xi \circ T_{0} \theta\right)}(g \circ \theta)=O_{(0, \xi)}(g)$.

To state the invariance property of the symbol, we need to specify the action of diffeomorphisms on the symbol spaces of Definition 1.3.4. First of all, if $\theta: \mathbf{R}^{n} \rightarrow \mathbf{R}^{n}$ is a diffeomorphism with $\theta(0)=0$, then we let $\theta$ act on $\mathcal{D}^{\prime}\left(\mathbf{R}^{n}\right)$ by $h \mapsto h \circ T_{0} \theta$. This action preserves bounded sets, so it lifts to an action on $S^{N}\left(\mathcal{Q}^{\prime}\left(\mathbf{R}^{n}\right)\right) / S^{N-1 / 2}\left(\mathcal{D}^{\prime}\left(\mathbf{R}^{n}\right)\right)$. On the other hand, $\theta$ induces a map from $\mathscr{g}_{(0, \xi)}$ to $\mathcal{G}_{\left(0, \xi \circ T_{0} \theta\right)}$ by $[\varphi] \mapsto[\varphi \circ \theta]$. (This map depends upon the second derivative of $\theta$ at 0 .) Composition with these actions of $\theta$ gives a map from $\delta_{(0, \xi)}^{N}\left(\mathbf{R}^{n}\right)$ to $\delta_{\left(0, \xi \circ T_{0} \theta\right)}^{N}\left(\mathbf{R}^{n}\right)$, which we denote by $\sigma_{(0, \xi)}(\theta) .\left(\sigma_{(0, \xi)}(\theta)\right.$ can actually be identified with the symbol in our sense of the Schwartz kernel of the operator $\circ \theta$.) Chasing through the definitions gives the following result.

Corollary 1.4.3 Let $O_{(0, \xi)}(g) \leqslant N, \theta: \mathbf{R}^{n} \rightarrow \mathbf{R}^{n}$ a diffeomorphism with $\theta(0)=0$. Then

$$
\left.u_{(0, \xi} \wedge T_{0} \theta\right)(g \circ \theta)=o_{(0, \xi)}(\theta)\left[\sigma_{(0, \xi)}(g)\right] .
$$

\subsection{Multiplication by $C^{\infty}$ functions, tensor products.}

Proposition 1.5.1. Let $p$ be a complex-valued $C^{\infty}$ function vanishing to order $\geqslant k$ at 0 . Suppose $O_{(0, \xi)}(g) \leqslant N$. Then.

(a) $O_{(0, \xi)}(p g) \leqslant N-k / 2$;

(b) if $p(x)=\sum_{|\alpha|=k} c_{\alpha} x^{\alpha}+q(x)$, where $q$ vanishes to order $\geqslant k+1$ at 0 , then

$$
\sigma_{(0, \xi)}^{N-k / 2}(p g)=\tau^{k / 2}\left(\sum_{|\alpha|=k} c_{\alpha} x^{\alpha}\right) \sigma_{(0, \xi)}^{N}(g) .
$$

(The symbol space $\varsigma_{(0, \xi)}^{N}\left(\mathbf{R}^{n}\right)$ is a module over $C^{\infty}\left(\mathbf{R}^{n}\right)$. See the discussion following Definition 1.3.3.)

Proof. (a) We have

$$
\begin{aligned}
\tau^{-n / 2}\left\langle(p g)_{\varphi}^{\tau}, u\right\rangle & =\int g(x) e^{-i \tau \varphi(x)} p(x) u(\sqrt{\tau} x) d x \\
& =\int g(x) e^{-i \tau \varphi(x)} v_{u}^{\tau}(\sqrt{\tau} x) d x
\end{aligned}
$$

where $v_{u}(x)=p(x / \sqrt{\tau}) u(x)$. As usual, we need to show that $\left\{\tau^{k / 2} v_{u}^{\tau} \mid \tau \geqslant\right.$ $1, u \in A\}$ is bounded whenever $A$ is bounded. Writing $p(x)=\sum_{|\alpha|=k} p_{\alpha}(x) x^{\alpha}$, 
we have

$$
p_{\alpha}\left(\frac{x}{\sqrt{\tau}}\right)=\tau^{-k / 2} \sum_{|\alpha|=k} p_{\alpha}\left(\frac{x}{\sqrt{\tau}}\right) x^{\alpha}
$$

so

$$
\tau^{k / 2} v_{u}^{\tau}(x)=\left(\sum_{|\alpha|=k} p_{\alpha}\left(\frac{x}{\sqrt{\tau}}\right) x^{\alpha}\right) u(x),
$$

which is obviously bounded.

(b) By (a), it suffices to check (1.5.1) for the case where $p(x)=\sum_{|\alpha|=k} c_{\alpha} x^{\alpha}$. But, in this case $(p g)_{\varphi}^{\tau}$ is equal to $\tau^{-k / 2} p g_{\varphi}^{\tau}$.

REMARK 1.5.2. With $p$ as in Proposition 1.5.1, we have by Example 1.3.8 that $O_{(0,0)}(p) \leqslant-k / 2$, and $\sigma_{(0,0)}^{-k / 2}(p)=\tau^{-k / 2} \sum_{|\alpha|=k} c_{\alpha} x^{\alpha}$ (identifying $\tilde{D}^{\prime}$ with $\mathscr{O}$ by choosing the 2-jet of the zero function in $\left.g_{(0,0)}\left(\mathbf{R}^{n}\right)\right)$. Therefore, we may write (1.5.1) in the form

$$
\sigma_{(0, \xi)}^{N-k / 2}(p g)=\left(\sigma_{(0,0)}^{-k / 2}(p)\right)\left(\sigma_{(0, \xi)}^{N}(g)\right)
$$

This suggests that Proposition 1.5.1 should be a special case of a result valid for the product of two distributions, whenever that product exists. We suspect that the correct expression should be a "convolution" of the form

$$
\sigma_{(0, \xi)}\left(g_{1} g_{2}\right)=\int\left(\sigma_{(0, \eta)}\left(g_{1}\right)\right)\left(\sigma_{(0, \xi-\eta)}\left(g_{2}\right)\right) d \eta \text {. }
$$

We are still far from making sense out of 1.5 .3 , much less proving it-here are some of the difficulties.

(a) It is not clear at what orders the symbols are to be taken.

(b) To obtain the integrand, we must multiply elements of $\delta_{(0, \eta)}$ and $\delta_{(0, \xi-\eta)}$ to obtain an element of $\delta_{(0, \xi)}$. Formally, this presents no problem since addition of functions induces a natural operation from $g_{(0, \xi)}\left(\mathbf{R}^{n}\right) \times$ $g_{(0, \xi-\eta)}\left(\mathbf{R}^{n}\right)$ to $g_{(0, \xi)}\left(\mathbf{R}^{n}\right)$. However, in multiplying the two symbols together, we are essentially multiplying distributions, and it may be necessary to assume, as an additional hypothesis, that this multiplication is possible.

(c) How does one make sense out of the integral in (1.5.3)? It is necessary to understand the behavior of $\sigma_{(0, \xi)}(g)$ as a function of $\xi$. Should it really be considered as some kind of distribution in $\xi$ ?

Problem 1.5.3. It can occur that the product on the right-hand side of (1.5.1) is zero, even if both factors $\sum_{|\alpha|=k} c_{\alpha} x^{\alpha}$ and $\sigma_{(0, \xi)}^{N}(g)$ are nonzero. In this case, we may conclude that $O_{(0, \xi)}(p g) \leqslant N-k / 2-1 / 2$. Find a formula for $\sigma_{(0, \xi)}^{N-k / 2-1 / 2}(p g)$. It will have to depend upon the terms of order $k+1$ in the Taylor expansion of $p$, as well as the "term of order $N-1 / 2$ in the asymptotic expansion of $g_{\varphi}^{\tau}$."

The order and symbol behave in a very simple way with respect to the 
tensor product of distributions. We need only to describe some natural mappings. We write $g_{1} \otimes g_{2}$, or $g_{1}(x) g_{2}(y)$ for the tensor product, in $\mathscr{D}^{\prime}\left(\mathbf{R}^{n_{1}+n_{2}}\right)$, of $g_{1} \in \mathscr{D}^{\prime}\left(\mathbf{R}^{n_{1}}\right)$ and $g_{2} \in \mathscr{D}^{\prime}\left(\mathbf{R}^{n_{2}}\right)$. This operation induces in a natural way products, which we also denote by $\otimes$, from $S^{N_{1}}\left(\mathscr{D}^{\prime}\left(\mathbf{R}^{n_{1}}\right)\right) \times$ $S^{N_{2}}\left(\mathscr{D}^{\prime}\left(\mathbf{R}^{n_{2}}\right)\right)$ to $S^{N_{1}+N_{2}}\left(\mathscr{D}^{\prime}\left(\mathbf{R}^{n_{1}+n_{2}}\right)\right)$ and from

$$
S^{N_{1}}\left(\mathscr{D}^{\prime}\left(\mathbf{R}^{n_{1}}\right)\right) / S^{N_{1}-1 / 2}\left(\mathscr{D}^{\prime}\left(\mathbf{R}^{n_{1}}\right)\right) \times S^{N_{2}}\left(\mathscr{D}^{\prime}\left(\mathbf{R}^{n_{2}}\right)\right) / S^{N_{2}-1 / 2}\left(\mathscr{D}^{\prime}\left(\mathbf{R}^{n_{2}}\right)\right)
$$

to

$$
S^{N_{1}+N_{2}}\left(\mathcal{D}^{\prime}\left(\mathbf{R}^{n_{1}+n_{2}}\right)\right) / S^{N_{1}+N_{2}-1 / 2}\left(\mathcal{D}^{\prime}\left(\mathbf{R}^{n_{1}+n_{2}}\right)\right) \text {. }
$$

On the other hand, we have a natural identification of $\mathbf{R}^{n_{i}^{*}} \times \mathbf{R}^{n_{2}^{*}}$ with $\left(R^{n_{1}+n_{2}}\right)^{*}$, and the operation

$$
\left(\varphi_{1}(x), \varphi_{2}(y)\right) \mapsto\left(\varphi_{1}(x)+\varphi_{2}(y)\right)
$$

then induces an injection of $J_{(0, \xi)}\left(\mathbf{R}^{n_{1}}\right) \times J_{(0, \eta)}\left(\mathbf{R}^{n_{2}}\right)$ into $J_{(0,(\xi, \eta))}\left(\mathbf{R}^{n_{1}+n_{2}}\right)$. Putting all these mappings together, we obtain a mapping

$$
\mathcal{S}_{(0, \xi)}^{N_{1}}\left(\mathbf{R}^{n_{1}}\right) \times \mathcal{S}_{(0, \eta)}^{N_{2}}\left(\mathbf{R}^{n_{2}}\right) \stackrel{\otimes}{\rightarrow} \mathcal{S}_{(0(\xi, \eta))}^{N}\left(\mathbf{R}^{n_{1}+n_{2}}\right) .
$$

We leave the proof of the following to the reader.

Proposition 1.5.4. Let $g_{1} \in \mathscr{D}^{\prime}\left(\mathbf{R}^{n_{1}}\right), O_{(0, \xi)}\left(g_{1}\right) \leqslant N_{1} ; g_{2} \in \mathscr{Q}^{\prime}\left(\mathbf{R}^{n_{2}}\right)$, $O_{(0, \eta)}\left(g_{2}\right)<N_{2}$. Then $O_{(0,(\xi, \eta))}\left(g_{1} \otimes g_{2}\right) \leqslant N_{1}+N_{2}$, and

$$
\sigma_{(0,(\xi, \eta))}^{N_{1}+N_{3}}\left(g_{1} \otimes g_{2}\right)=\sigma_{(0, \xi)}^{N_{1}}\left(g_{1}\right) \otimes \sigma_{(0, \eta)}^{N_{2}}\left(g_{2}\right) \text {. }
$$

Remark 1.5.5. Proposition 1.5.4 shows that, even if one is in the end interested only in the behavior of distributions for $\xi \neq 0$, it is still useful to understand the case $\xi=0$.

EXAMPLE 1.5.6. Let $g \in \mathscr{D}^{\prime}\left(\mathbf{R}^{2}\right)$ be the characteristic function of a region in the plane bounded by a piecewise smooth curve. Suppose that the origin is at a "corner" of this curve; i.e. the tangents to the two pieces of the curve at the origin are neither parallel nor antiparallel. After applying a diffeomorphism, an operation whose effect on the order and symbol is easily calculated from Corollary 1.4.3 and Proposition 1.5.1, we may bring $g$ locally into the form

$$
g\left(x_{1}, x_{2}\right)=\left\{\begin{array}{cc}
1, & 0 \leqslant x_{1}, 0 \leqslant x_{2} \\
0, & \text { otherwise }
\end{array}\right\},
$$

i.e. $g=\delta^{(-1)} \otimes \delta^{(-1)}$, where $\delta^{(-1)}$ is the Heaviside function.

By the result mentioned at the end of Example 1.3.10, combined with Proposition 1.5.4, we have

$$
O_{\left(0,\left(\xi_{1}, \xi_{2}\right)\right)}(g)= \begin{cases}0, & \xi_{1}=0, \xi_{2}=0 \\ -\frac{1}{2}, & \xi_{1}=0, \xi_{2} \neq 0 \text { or } \xi_{1} \neq 0, \xi_{2}=0 \\ -1, & \xi_{1} \neq 0, \xi_{2} \neq 0 .\end{cases}
$$


For the symbols we have (modulo the usual identifications)

$$
\sigma_{\left(0,\left(\xi_{1}, \xi_{2}\right)\right)}^{N}(g)=\left\{\begin{array}{lll}
\delta^{(-1)} \otimes \delta^{(-1)}, & \xi_{1}=\xi_{2}=0, & N=0, \\
\tau^{-1 / 2} \delta^{(-1)} \otimes \delta^{(0)}, & \xi_{1}=0, \xi_{2} \neq 0, & N=-1 / 2, \\
\tau^{-1 / 2} \delta^{(0)} \otimes \delta^{(-1)}, & \xi_{1} \neq 0, \xi_{2}=0, & N=-1 / 2, \\
\tau^{-1} \delta^{(0)} \otimes \delta^{(0)}, & \xi_{1} \neq 0, \xi_{2} \neq 0, & N=-1 .
\end{array}\right.
$$

Going back to the original $g$, we may conclude, for instance, that the order with respect to a $\xi$ which is normal to neither of the incoming edges is -1 , and that the symbol of order -1 with respect to a suitably chosen element of $J_{(0, \xi)}$ is equal to $\tau^{-1}$ times the delta function at the origin.

It should be interesting to analyze cases where the boundary of the region has more complicated singularities such as cusps.

1.6. Vector-valued distributions and distributions on manifolds. Let $V$ be a finite-dimensional vector space over $\mathbf{C}$. The space $\mathscr{D}^{\prime}\left(\mathbf{R}^{n} ; V\right)$ of $V$-valued distributions on $\mathbf{R}^{n}$ is defined as the dual space to the space $\mathscr{D}\left(\mathbf{R}^{n} ; V^{*}\right)$ of compactly supported $C^{\infty}$ functions on $\mathbf{R}^{n}$ with values in $V^{*}$. There are natural identifications

$$
\mathscr{D}^{\prime}\left(\mathbf{R}^{n} ; V\right) \approx \mathscr{D}^{\prime}\left(\mathbf{R}^{n}\right) \otimes_{\mathrm{C}} V, \quad \mathscr{D}\left(\mathbf{R}^{n} ; V^{*}\right) \approx \mathscr{D}\left(\mathbf{R}^{n}\right) \otimes_{\mathrm{C}} V^{*} .
$$

Everything which we have done up to now may be repeated for vector-valued distributions. Whenever the notation $\left(\mathbf{R}^{n}\right)$ occurs in the name of a space of distributions or symbols we create a new space by tensoring with $V$ and replacing the notation $\left(\mathbf{R}^{n}\right)$ by $\left(\mathbf{R}^{n} ; V\right)$. A little extra must be said regarding products. In Proposition 1.5.1, if $g \in \mathscr{D}^{\prime}\left(\mathbf{R}^{n} ; V\right)$, we can allow $p$ to take values in the space $\operatorname{Hom}(V, W)$, where $W$ is another vector space; $p g$ then belongs to $\mathscr{D}^{\prime}\left(\mathbf{R}^{n} ; W\right)$. In Proposition 1.5.4, if $g_{1} \in \mathscr{D}^{\prime}\left(\mathbf{R}^{n_{1}} ; V_{1}\right)$ and $g_{2} \in$ $\mathcal{D}^{\prime}\left(\mathbf{R}^{n_{2}} ; V_{2}\right), g_{1} \otimes g_{2}$ belongs to $\mathscr{D}^{\prime}\left(\mathbf{R}^{n_{1}+n_{2}} ; V_{1} \otimes V_{2}\right)$.

Next, we may replace $\mathbf{R}^{n}$ by any of its open sets. Namely, if $\mathscr{V} \subseteq \mathbf{R}^{n}$ is open, $\mathscr{D}(\mathfrak{V})$ is the subspace of $\mathscr{D}\left(\mathbf{R}^{n}\right)$ consisting of functions whose support is contained in $\mathcal{V}$. The dual space $\mathscr{D}(\mathcal{V})$ is denoted by $\mathscr{D}(\mathcal{V})$; its elements are called distributions on $\mathfrak{V}$. If $\mathscr{V} \subseteq \mathscr{W}$, the inclusion $\mathscr{D}(\mathfrak{V}) \rightarrow \mathscr{D}(\mathcal{W})$ induces a restriction mapping from $\mathscr{D}^{\prime}(\mathscr{W})$ to $\mathscr{D}^{\prime}(\mathcal{V})$. If $\mathcal{V}$ contains the origin, we may define $O_{(0, \xi)}(g)$ and $\sigma_{(0, \xi)}^{N}(g)$ for $g \in \mathscr{D}^{\prime}(\mathcal{V})$ as follows. Choose a $C^{\infty}$ function $\mathcal{X}$ on $\mathbf{R}^{n}$ with support in $\mathcal{V}$ such that $\mathcal{X} \equiv 1$ on a neighborhood of the origin. Now $\mathcal{X g}$ is the restriction of an element $\bar{g} \in \mathscr{D}^{\prime}\left(\mathbf{R}^{n}\right)$; we may define the order and symbol of $g$ to be the order and symbol of $\bar{g}$ in the usual sense. This is independent of $\mathcal{X}$ because the order and symbol depend only upon local behavior (Lemma 1.1.5). Notice that, although $g$ is defined only on $\mathcal{T}$, the symbol $\sigma_{(0, \xi)}^{N}(g)$ still lies in $\mathcal{S}_{(0, \xi)}^{N}\left(\mathbf{R}^{n}\right)$.

Of course, we can combine the two generalizations just made, defining 
$V$-valued distributions on $\mathcal{V}$ and their orders and symbols.

If $\mho_{1}$ and $\mho_{2}$ are open subsets of $\mathbf{R}^{n}$, and $V_{1}$ and $V_{2}$ are vector spaces, a diffeomorphism

$$
\Theta: \mathscr{V}_{1} \times V_{1} \rightarrow \widetilde{V}_{2} \times V_{2}
$$

is called a bundle isomorphism if the first component of $\Theta(x, v)$ depends only on $x$, and the second component depends linearly upon $v$ for each fixed $x$. We may write, therefore, $\Theta(x, v)=(\theta(x), p(x) \cdot v)$, where $\theta$ is a diffeomorphism from $\mho_{1}$ to $\mho_{2}$ and $p$ is a $C^{\infty}$ map from $\mho_{1}$ to $\operatorname{Hom}\left(V_{1}, V_{2}\right)$. Using $\Theta$ we may define a pullback operation

$$
\Theta^{*}: \mathscr{D}^{\prime}\left(\mathscr{V}_{2}, V_{2}\right) \rightarrow \mathscr{D}^{\prime}\left(\mathscr{V}_{1}, V_{1}\right)
$$

in the following manner. If $u \in \mathscr{D}\left(\mathscr{V}_{1}, V_{1}\right)$, we define $\Theta_{*} u \in \mathscr{D}\left(\mathscr{V}_{2}, V_{2}\right)$ as the function whose graph is the image under $\Theta$ of the graph of $u$; i.e.

$$
\left(\Theta_{*} u\right)(x)=p\left(\theta^{-1}(x)\right) \cdot u\left(\theta^{-1}(x)\right) \text {. }
$$

Now, for $g \in \mathscr{D}^{\prime}\left(\mathcal{V}_{2}, V_{2}\right)$, we define $\Theta^{*} g$ by

$$
\left\langle\Theta^{*} g, u\right\rangle=\left\langle g, J_{\theta^{-1}} \cdot \Theta_{*} u\right\rangle .
$$

(If $V_{1}=V_{2}$ and $p$ is the identity matrix, this reduces to (1.4.1).)

Combining Corollaries 1.4.2 and 1.4.3 with Proposition 1.5.1, we can describe the effect of pullback by a bundle isomorphism on the order and symbol.

Proposition 1.6.1. Let $\Theta(x, v)=(\theta(x), p(x) \cdot v)$ be a bundle map from $\mathfrak{V}_{1} \times V_{1}$ to $\mathfrak{V}_{2} \times V_{2}, g \in \mathscr{D}^{\prime}\left(\mathcal{V}_{2} ; V\right), \xi \in \mathbf{R}^{n^{*}}$. Suppose that $\mathfrak{T}_{1}$ and $\mathfrak{V}_{2}$ contain the origin, and $\theta(0)=0$. Then:

(i) $O_{\left(0, \xi \circ T_{0} \theta\right)}\left(\Theta^{*} g\right)=O_{(0, \xi)}(g)$;

(ii) $O_{(0, \xi)}(g) \leqslant N$ implies that $\sigma_{\left(0, \xi \circ T_{0} \theta\right)}\left(\Theta^{*} g\right)=\sigma_{(0, \xi)}(\Phi)\left[\sigma_{(0, \xi)}(g)\right]$, where $\sigma_{(0, \xi)}(\Phi)$ from

$$
\delta_{(0, \xi)}^{N}\left(\mathbf{R}^{n}, V_{2}\right) \approx \delta_{(0, \xi)}^{N}\left(\mathbf{R}^{n}\right) \otimes V_{2}
$$

to

$$
\delta_{\left(0, \xi \circ T_{0} \theta\right)}^{N}\left(\mathbf{R}^{n} ; V_{1}\right) \approx \delta_{\left(0, \xi \circ T_{0} \theta\right)}^{N}\left(\mathbf{R}^{n}\right) \otimes V_{1}
$$

is the tensor product of $\sigma_{(0, \xi)}(\theta): \mathcal{S}_{(0, \xi)}^{N}\left(\mathbf{R}^{n}\right) \rightarrow \mathcal{S}_{\left(0, \xi \circ T_{0} \theta\right)}^{N}\left(\mathbf{R}^{n}\right)$ (see the discussion before Corollary 1.4.3) with $p^{-1}(0): V_{2} \rightarrow V_{1}$.

We can now go on to discuss distributions on manifolds. Let $X$ be a paracompact $C^{\infty}$ manifold, $\mathbf{E} \stackrel{\pi}{\rightarrow} X$ a complex vector bundle. A chart for $\mathbf{E}$ consists of an open subset $\mathcal{Q} \subseteq X$ and a diffeomorphism

$$
\Psi: \pi^{-1}(\mathcal{Q}) \rightarrow \mho \times V
$$

where $\mathcal{V}$ is an open set in an euclidean space, $V$ is a vector space, the first component of $\Psi(\mathrm{v})$ depends only upon $\pi(\mathrm{v})$, and the second component of 
$\Psi(\mathbf{v})$ depends linearly upon $\mathbf{v}$ for $\pi(\mathbf{v})$ fixed. We denote by $\psi: \mathcal{U} \rightarrow \mathcal{V}$ the diffeomorphism for which the first component of $\Psi$ is $\psi \circ \pi$. Given charts $\Psi_{\alpha}$ and $\Psi_{\beta}$ for $\mathbf{E}$, the diffeomorphism

$$
\Theta_{\beta \alpha}: \psi_{\alpha}\left(\mathcal{U}_{\alpha} \cap \mathcal{Q}_{\beta}\right) \times V_{\alpha} \rightarrow \psi_{\beta}\left(\mathcal{U}_{\alpha} \cap \mathcal{Q}_{\beta}\right) \times V_{\beta}
$$

defined by $\Psi_{\beta} \circ \Psi_{\alpha}^{-1}$ is a bundle isomorphism.

We may now define an $\mathbf{E}$-valued distribution on $X$ to be a rule $g$ which assigns to each chart $\Psi_{\alpha}$ for $\mathbf{E}$ a distribution $g_{\alpha} \in \mathscr{D}^{\prime}\left(\mathscr{V}_{\alpha}, V_{\alpha}\right)$ in such a way that, if $\Psi_{\alpha}$ and $\Psi_{\beta}$ are any two charts, then the pullback operator $\Theta_{\beta \alpha}^{*}$ maps the restriction of $g_{\beta}$ to $\psi_{\beta}\left(\mathcal{U}_{\alpha} \cap \mathcal{U}_{\beta}\right)$ to the restriction of $g_{\alpha}$ to $\psi_{\alpha}\left(\mathcal{Q}_{\alpha} \cap \mathcal{U}_{\beta}\right)$. The space of $\mathbf{E}$-valued distributions on $X$ is denoted by $\mathscr{D}^{\prime}(X ; \mathbf{E})$.

There is also a global definition of distributions on manifolds. If $E$ is any vector bundle over $X$, we denote by $\mathscr{D}(X ; E)$ the space of compactly supported $C^{\infty}$ sections of $E$; a subset $A$ of $\mathscr{D}(X ; E)$ is bounded if all its elements are supported in a fixed compact set $K$ and if, with respect to a finite set of coordinate systems covering $K$, the partial derivatives of each order of the elements of $A$ are uniformly bounded. Let $\Omega_{1} X$ be the bundle of 1-densities on $X$ (see $\$ 3.3$ or p. 14 of [2]). Each smooth section of $E$ defines a linear functional on $\mathscr{D}\left(X, E^{*} \otimes \Omega_{1} X\right)$ which is bounded on bounded sets; in fact, if $g$ is a section of $E$ and $u \in \mathscr{D}\left(X, E^{*} \otimes \Omega_{1} X\right)$, we may pair $g$ with $u$ to obtain an element $(g, u)$ of $\mathscr{D}\left(\Omega_{1} X\right)$. Integrating $(g, u)$ over $X$ gives a complex number $\langle g, u\rangle$. We may define $\mathscr{D}^{\prime}(X ; E)$ to be the space of all linear functionals on $\mathscr{D}\left(X ; E^{*} \otimes \Omega_{1}\right)$ which are bounded on bounded sets. It is not hard to check that this definition agrees with the previous one. (The densities are implicitly contained in the previous definition because of the Jacobian factor in the pullback formula (1.6.1).)

Now let $g$ be an $\mathbf{E}$-valued distribution on $X, x$ a point of $X$, and $\xi \in T_{x}^{*} X$ a cotangent vector at $x$. Given any chart $\Psi_{\alpha}$ for $\mathbf{E}$ such that $\psi_{\alpha}(x)=0$, $\boldsymbol{\xi} \circ T_{0} \psi^{-1}$ is a cotangent vector $\left(0, \xi_{\alpha}\right)$ at the origin in $\mathbf{R}^{n}$, so the order $O_{\left(0, \xi_{\alpha}\right)}\left(g_{\alpha}\right)$ is defined. It is easy to check that $\xi_{\alpha}=\xi_{\beta} \circ T_{0} \theta_{\beta \alpha}$, so by Proposition 1.6.1 we have, restricting to the images of $\mathcal{U}_{\alpha} \cap \mathcal{Q}_{\beta}$,

$$
O_{\left(0, \xi_{\alpha}\right)}\left(g_{\alpha}\right)=O_{\left(0, \xi_{\beta} \circ T_{0} \theta_{\beta \alpha}\right)}\left(\Theta_{\beta \alpha}^{*} g_{\beta}\right)=O_{\left(0, \xi_{\beta}\right)}\left(g_{\beta}\right) .
$$

This justifies the following definition.

Definition 1.6.2. Let $g \in \mathbb{D}^{\prime}(X ; \mathbf{E}), x \in X, \xi \in T_{x}^{*} X$. Then $O_{\xi}(g)$ is defined to be $O_{\left(0, \xi_{\alpha}\right)}\left(g_{\alpha}\right)$, where $\Psi_{\alpha}$ is any chart for $\mathbf{E}$ such that $\psi_{\alpha}(x)=0$.

Next we define the space $\delta_{\xi}^{N}(X ; E)$ in which the symbol $\sigma_{\xi}^{N}(g)$ will lie. The transformation laws in Corollary 1.4.3 and Proposition 1.6.1 show that the symbol should live on the tangent space $T_{x} X$ and should have values in the fibre $E_{x}$ of $\mathbf{E}$ over $x$.

To construct the "twisted" spaces at $\xi$, we consider the space $g_{\xi}(X)$ of 2-jets of real valued $C^{\infty}$ functions $\varphi$ on $X$ such that $\varphi(x)=0$ and $d \varphi(x)=\xi$, 
two functions $\varphi_{1}$ and $\varphi_{2}$ being considered equivalent if $\varphi_{1}-\varphi_{2}$ vanishes to order at least three at $x$. As in $\S 1.3$, we can show that $g_{\xi}(X)$ is an affine space modeled on the vector space of quadratic functions on $T_{x} X$, and that the elements of $g_{\xi}(X)$ can be identified with those lagrangian subspaces of the tangent space $T_{\xi} T^{*} X$ which are transversal to the fibre. Imitating Definition 1.3.3, we define, for each $C^{\infty}\left(T_{x} X\right)$-module $\mathcal{E}$, the twisted space $\tilde{\varepsilon}_{\xi}$ consisting of the mappings $[\varphi] \mapsto g_{[\varphi]}$ from $g_{\xi}(X)$ to $\mathcal{E}$ such that $g_{[\varphi]+q}=e^{-i q} g_{[\varphi]}$ for each quadratic function $q$ (the "sum" $[\varphi]+q$ refers to the affine structure on $\left.g_{\xi}(X)\right)$.

Finally, we define $\mathcal{S}_{\xi}^{N}(X)$ to be

$$
\left.\widehat{S^{N}\left(\mathscr{D}^{\prime}\left(T_{x} X\right)\right) / S^{N-1 / 2}\left(\mathscr{D}^{\prime}\left(T_{x} X\right)\right)}\right]_{\xi}
$$

and $\delta_{\xi}^{N}(X ; E)$ to be $\delta_{\xi}^{N}(X) \otimes E_{x}$.

Any chart $\Psi_{\alpha}$ for $\mathbf{E}$ such that $\psi_{\alpha}(x)=0$ induces in a canonical way an isomorphism $\sigma_{\xi} \Psi_{\alpha}$ from $\delta_{\xi}^{N}(X ; \mathbf{E})$ to $\mathcal{S}_{(0, \xi)}^{N}\left(\mathbf{R}^{n} ; V_{\alpha}\right)$. We leave it to the reader to write down the explicit definition of this isomorphism and to check that, if $\Psi_{\alpha}$ and $\Psi_{\beta}$ are two such charts, then

$$
\sigma_{\xi} \Psi_{\alpha}=\left[\sigma_{\left(0, \xi_{\alpha}\right)} \Theta_{\beta \alpha}\right]^{-1 \cdot} \circ \sigma_{\xi} \Psi_{\beta} .
$$

Proposition 1.6.1 (b) now applies to justify the following definition.

Definition 1.6.3. Let $g \in \mathscr{D}^{\prime}(X, \mathrm{E}), x \in X, \xi \in T_{x}^{*} X, O_{\xi}(g)<N$. Then $\sigma_{\xi}^{N}(g) \in \mathcal{S}_{\xi}^{N}(X ; E)$ is defined to be $\left(\sigma_{\xi} \Psi_{\alpha}\right)^{-1}\left[\sigma_{\left(0, \xi_{\alpha}\right)}^{N}\left(g_{\alpha}\right)\right]$, where $\Psi_{\alpha}$ is any chart for $\mathbf{E}$ such that $\psi_{\alpha}(x)=0$.

REMARK 1.6.4. If for some $\psi_{\alpha}$ with $\psi_{\alpha}(x)=0, g_{\alpha}$ is homogeneous of degree $N$ at $\left(0, \xi_{\alpha}\right)$ (see Definition 1.3.12), then this is true for all such $\alpha$, and we say that $g$ is homogeneous of order $N$ at $\xi$. In this case, the homogeneous $N$-symbol of $g$ is an element of the twisted space $\operatorname{DD}^{\prime}\left(T_{x} X\right) \otimes E_{x}$ of mappings assigning an $E_{x}$-valued distribution on $T_{x} X$ to each element of $J_{\xi}(X)$.

EXAMPLE 1.6.5. Let $Y \subseteq X$ be a submanifold of codimension $r, \delta \in \mathscr{D}^{\prime}(X)$ a smooth delta function along $Y$; i.e. given any chart $\psi_{\alpha}$ such that the image of $Y$ is defined by $x_{1}=\cdots=x_{r}=0, \delta_{\alpha}$ has the form

$$
a\left(x_{r+1}, \ldots, x_{n}\right) \delta\left(x_{1}\right) \cdots \delta\left(x_{r}\right) \text {, }
$$

where $a$ is a smooth function.

Applying Example 1.3.9 and Proposition 1.5.1, we conclude the following for $x \in Y$ and $\xi \in T_{x}^{*} X$.

(i) $O_{\xi}(\delta)=-\infty$ if $\xi$ does not belong to the conormal bundle of $Y$.

(ii) If $\xi$ does belong to the conormal bundle of $Y$, then $\delta$ is homogeneous of order $r / 2$ at $\xi$; the homogeneous $N$-symbol of $\delta$ at $\xi$ assigns to certain distinguished elements (see (iii) below) of $g_{\xi}(X)$ a smooth delta function along $T_{x} Y \subseteq T_{x} X$ which is invariant under translations in the direction of $T_{x} Y$. 
(iii) The distinguished elements of $g_{\xi}(X)$ are the 2-jets of functions $\varphi$ such that $d \varphi(x)=\xi$ and $\varphi$ is identically zero on $Y$. The corresponding lagrangian subspaces of $T_{\xi}\left(T^{*} X\right)$ are those which have the maximal intersection (of dimension $n-r$ ) with the tangent space at $\xi$ to the conormal bundle of $Y$.

In Theorem 3.3.7, we will see that this description of the symbol of a smooth delta function remains valid for any Fourier integral distribution-the conormal bundle of $Y$ being replaced by an arbitrary conic lagrangian submanifold of $T^{*} X$.

\section{Pseudodifferential Operators and the Wavefront SeT}

2.0. Introduction. The notion of localizing distributions in the cotangent bundle is closely tied to the action of pseudodifferential operators. Given a distribution $g \in \mathscr{D}^{\prime}(X)$, the common zero set of the principal symbols of pseudodifferential operators $P$ for which $P g$ is smooth is a closed conic subset of $\dot{T}^{*} X$ (the dot over the $T$ indicates that the zero section has been deleted), the so-called wavefront set WF $(g)$ of $g$. For each $\xi \in \dot{T}^{*} X$, the quotient of $D^{\prime}(X)$ by those distributions whose wavefront set does not contain $\xi$ is called the space of microfunctions at $\xi$; we denote it by $\Re_{\xi}(X)$. Since the projection into $X$ of $\operatorname{WF}(g)$ is the singular support of $g$ (see [6, Theorem 2.5.3]), it follows that $g$ is determined modulo $\mathcal{E}$ (the $C^{\infty}$ functions) by its image in all the $\Re_{\xi}(X)$ as $\xi$ runs over $\dot{T}^{*} X$. With this observation, we can identify $\mathscr{D}^{\prime}(X) / \mathcal{E}(X)$ with the global sections of the sheaf $\Re(X)$ over $\dot{T}^{*} X$ whose stalks are the $\mathfrak{M}_{\xi}(X)$.

The present work on the symbol of a distribution was intended partly as an attempt to obtain a "picture" of $\mathscr{R}(X)$ by representing its sections as sections of a vector bundle over $\dot{T}^{*} X$. The vector bundles $\mathcal{S}^{N}(X)$ (restricted to $\left.\dot{T}^{*} X\right)$ and the order and symbol defined in Chapter I accomplish this task to a certain extent. As we shall see in $\$ 2.2$ below, $O_{\xi}(g)=-\infty$ if $\xi \in T^{*} X$ is not in WF $(g)$; it follows that $O_{\xi}$ and $\sigma_{\xi}$ are defined on $\Re_{\xi}(X)$. The main tool for proving this fact is our Theorem 2.1.2 relating the order and symbol of $\mathrm{Pg}$ with that of $g$, where $P$ is a pseudodifferential operator. This result may be considered as a generalization of Proposition 1.5.1; of course the principal symbol of $P$ plays a crucial role.

Many of the details in the proof of Theorem 2.1.2 are due to K. Sklower, who found and filled several gaps in my own proof and then wrote the first draft of $\$ 2.1$.

2.1. Action of pseudodifferential operators. This section was written jointly with K. Sklower. We will follow the presentation of pseudodifferential operators given in $\$ 2.1$ of [6].

A pseudodifferential operator of order $m$ and type $(\rho, \delta)$ on $\mathbf{R}^{n}$ is defined by the formula 


$$
P g(x)=(2 \pi)^{-n} \int e^{i\langle\xi, x\rangle} p(x, \xi) \hat{g}(\xi) d \xi
$$

where

$$
\hat{g}(x)=\int e^{-i\langle\xi, x\rangle} g(x) d x
$$

and the total symbol $p(x, \xi)$ belongs to Hörmander's symbol class $S_{\rho, \delta}^{m}\left(\mathbf{R}^{n} \times\right.$ $\left.\mathbf{R}^{n^{*}}\right)$; i.e.

$$
\left(\frac{\partial}{\partial x}\right)^{\beta}\left(\frac{\partial}{\partial \xi}\right)^{\alpha} p(x, \xi)=O(1+|\xi|)^{m-\rho|\alpha|+\delta|\beta|}
$$

uniformly on compact sets of $x$, for each pair $(\alpha, \beta)$ of multi-indices. We will assume throughout this section that $0 \leqslant \delta \leqslant 1 / 2<\rho \leqslant 1$ and $\rho-\delta \geqslant 1 / 2$.

The following definition is not quite conventional.

DEFinition 2.1.1. If $P$ is a pseudodifferential operator with total symbol $p \in S_{\rho, \delta}^{m}$, a principal symbol for $P$ is an element $p_{1} \in S_{\rho, \delta}^{m}$ such that $p-p_{1} \in$ $S_{\rho, \delta}^{m-1 / 2}$.

For example, we will speak of an operator as having principal symbol of type $(1,0)$, or homogeneous principal symbol. In the latter case, there is an unique function $p_{1}(x, \xi)$, positively homogeneous of degree $m$ in $\xi$, such that $p-p_{1} \in S_{\rho, \delta}^{m-1 / 2}$ for large $\xi$. We will refer to this $p_{1}(x, \xi)$ as the homogeneous $m$-symbol of $P$.

The integrals in (2.1.1) and (2.1.2) are absolutely convergent if $g \in \mathscr{D}\left(\mathbf{R}^{n}\right)$, in which case $P g$ is $C^{\infty}$ but not necessarily compactly supported. If $P$ is in addition properly supported, i.e. the support of the Schwartz kernel of $P$ has proper projection into either factor $\mathbf{R}^{n}$, then $P$ maps $\mathscr{D}\left(\mathbf{R}^{n}\right)$ into $\mathscr{D}\left(\mathbf{R}^{n}\right)$, and this map is continuous since all the $x$-derivatives of $p(x, \xi)$ have polynomial growth. It follows that we have a transpose map $P^{t}: \mathscr{D}^{\prime}\left(\mathbf{R}^{n}\right) \rightarrow \mathscr{D}^{\prime}\left(\mathbf{R}^{n}\right)$ given by $\left\langle P^{\prime} g, u\right\rangle=\langle g, P u\rangle$ for $g \in \mathscr{D}^{\prime}\left(\mathbf{R}^{n}\right) u \in \mathscr{D}\left(\mathbf{R}^{n}\right)$.

Now it turns out (see p. 105 of [6]) that the restriction of $P^{t}$ to $\mathscr{D}\left(\mathbf{R}^{n}\right)$ is itself a properly supported pseudodifferential operator with total symbol $q \in S_{\rho, \delta}^{m}\left(\mathbf{R}^{n} \times \mathbf{R}^{n^{*}}\right)$. By formula (2.1.6) of [6], we have $q(x, \xi)=\rho(x,-\xi)$ modulo $S_{\rho, \delta}^{m-(\rho-\delta)}$, so $P^{t}$ has a principal symbol of type $(1,0)$ or a homogeneous principal symbol if and only if $P$ does. Finally, since $P^{t}$ maps $\mathscr{D}\left(\mathbf{R}^{n}\right)$ continuously to $\mathscr{D}\left(\mathbf{R}^{n}\right)$, we may extend $P$ itself to $\mathscr{D}\left(\mathbf{R}^{n}\right)$ by the formula

$$
\langle P g, u\rangle=\left\langle g, P^{t} u\right\rangle \quad g \in \mathscr{D}^{\prime}\left(\mathbf{R}^{n}\right), u \in \mathscr{D}\left(\mathbf{R}^{n}\right) .
$$

The remainder of this section will be devoted to the proof of the following theorem.

THEOREM 2.1.2. Let $P$ be a properly supported pseudodifferential operator of order $m$ and type $(\rho, \delta)$, where $0 \leqslant \delta \leqslant 1 / 2<\rho \leqslant 1$ and $\rho-\delta \geqslant 1 / 2$. Let $g \in \mathscr{D}^{\prime}\left(\mathbf{R}^{n}\right)$ and $0 \neq \xi \in \mathbf{R}^{n^{*}}$ such that $O_{(0, \xi)}(g) \leqslant N$. Then: 
(a) $O_{(0, \xi)}(P g) \leqslant N+m$;

(b) $\sigma_{(0, \xi)}^{N+m}(P g)$ depends only upon $\sigma_{(0, \xi)}^{N}(g)$ and the behavior of the total symbol $p$ on a conic neighborhood of $(0, \xi)$.

(c) If $P$ has a principal symbol $p_{1}$ of type $(1,0)$, then

$$
\sigma_{(0, \xi)}^{N+m}(P g)=p_{1}(0, \tau \xi) \sigma_{(0, \xi)}^{N}(g) \text {. }
$$

(d) If $P$ has homogeneous $m$-symbol $p_{h}$ and $g$ is homogeneous of order $N$ at $(0, \xi)$, then $\mathrm{Pg}$ is homogeneous of order $N+m$ at $(0, \xi)$, and the homogeneous $N+m$ symbol of $P g$ at $(0, \xi)$ equals $p_{h}(0, \xi)$ times the homogeneous $N$ symbol of $g$ at $(0, \xi)$.

Our first step in proving Theorem 2.1.2 is to observe that we may assume $g$ to be compactly supported. In fact, let $S \subseteq \mathbf{R}^{n} \times \mathbf{R}^{n}$ be the support of the Schwartz kernel $K(x, y)$ of $P$, i.e.

$$
P g(x)=\int K(x, y) g(y) d y .
$$

Choose any compact neighborhood $D$ of the origin in $\mathbf{R}^{n}$; since $P$ is properly supported, the set $E=\{y \mid(x, y) \in S$ for some $x \in D\}$ is also compact in $\mathbf{R}^{n}$. Now let $\mathcal{X} \in \mathscr{D}\left(\mathbf{R}^{n}\right)$ be identically 1 on a neighborhood of $E \cup\{0\}$. Then $\mathcal{X} g$ agrees with $g$ near the origin. On the other hand, $g-\mathfrak{X g}$ vanishes on a neighborhood of $E$, so $P g-P \mathscr{X g}$ vanishes on $D$, and $P g$ agrees with $P \mathscr{X g}$ in a neighborhood of the origin. Since the order and symbol are local invariants, by Lemma 1.1.5 and its proof, the statement of Theorem 2.1.2 remains unchanged if we replace $g$ by $\mathfrak{X}$.

From now on, then, we assume that $g$ is compactly supported and we analyze the asymptotic behavior of $\left\langle(P g)_{\varphi}^{\tau}, u\right\rangle$, where $u$ ranges over a bounded subset of $\mathscr{D}\left(\mathbf{R}^{n}\right)$. As usual, we will take $\varphi(x)=\langle\xi, x\rangle$ and write $g_{\xi}^{\tau}$ for $g_{\varphi}^{\tau}$. By using formulas (2.1.3) and (1.1.1), we will be able to write

$$
\left\langle(P g)_{\xi}^{\tau}, u\right\rangle=\left\langle g_{\xi}^{\tau}, v_{u}^{\tau}\right\rangle .
$$

The bulk of the proof will involve analyzing the family $v_{u}^{\tau}$.

First, we must obtain an explicit formula for $v_{u}^{\tau}$. We have

$$
\begin{aligned}
\left\langle(P g)_{\xi}^{\tau}, u\right\rangle & =\left\langle P g, \tau^{n / 2} e^{-i \tau\langle\xi, x\rangle} u(\sqrt{\tau} x)\right\rangle \\
& =\left\langle g, P^{t}\left[\tau^{n / 2} e^{-i \tau\langle\xi, x\rangle} u(\sqrt{\tau} x)\right]\right\rangle .
\end{aligned}
$$

A straightforward computation yields the Fourier transform formula

$$
\widehat{\tau^{n / 2} e^{-i \tau\langle\xi, x\rangle} u(\sqrt{\tau} x)}(\eta)=\hat{u}(\eta+\tau \xi / \sqrt{\tau}),
$$

so we have 


$$
\begin{aligned}
\left\langle(P g)_{\xi}^{\tau}, u\right\rangle & =\left\langle g,(2 \pi)^{-n} \int e^{i\langle\eta, x\rangle} q(x, \eta) \hat{u}(\eta+\tau \xi / \sqrt{\tau}) d \eta\right\rangle \\
= & \left\langle g,(2 \pi)^{-n} \tau^{n / 2} \int e^{i\langle\sqrt{\tau} \eta-\tau \xi, x\rangle} q(x, \sqrt{\tau} \eta-\tau \xi) \hat{u}(\eta) d \eta\right\rangle \\
= & \left\langle g, \tau^{n / 2} e^{i \tau\langle\xi, x\rangle}(2 \pi)^{-n} \int e^{i\langle\eta, \sqrt{\tau} x\rangle} q(x, \sqrt{\tau} \eta-\tau \xi) \hat{u}(\eta) d \eta\right\rangle,
\end{aligned}
$$

so (2.1.4) holds if we define

$$
v_{u}^{\tau}(x)=(2 \pi)^{-n} \int e^{i\langle\eta, x\rangle} q\left(\frac{x}{\sqrt{\tau}}, \sqrt{\tau} \eta-\tau \xi\right) \hat{u}(\eta) d \eta .
$$

Our immediate goal will be to establish part (a) of Theorem 2.1.2 concerning the order of $\mathrm{Pg}$. Having done this, we will know that the symbol of $\mathrm{Pg}$ depends only on the symbol of $g$ and the principal symbol of $P$-using this fact we can modify $P$ to simplify the proof of the rest of the theorem.

We must prove that $\tau^{-(N+m)}\left\langle(P g)_{\xi}^{\tau}, u\right\rangle$ is bounded, uniformly for $\tau \geqslant 1$ and $u$ in a bounded subset of $\mathscr{D}\left(\mathbf{R}^{n}\right)$. Using (2.1.4), we can rewrite this as $\left\langle\tau^{-N} g_{\xi}^{\tau}, \tau^{-m} v_{u}^{\tau}\right\rangle$; since $\tau^{-N} g_{\xi}^{\tau}$ is bounded, we would be done if we could show that $\tau^{-m} v_{u}^{\tau}$ is bounded. Due to the presence of $x / \sqrt{\tau}$ in (2.1.5), the supports of the $v_{u}^{\tau}$ are not necessarily contained in a fixed compact set; what we can do is to show that, for a fixed cutoff function $\mathcal{X}, \tau^{-m} \mathcal{X} v_{u}^{\tau}$ remains bounded. This will leave the task of estimating $\left\langle g_{\xi}^{\tau}\right.$, $\left.(1-\mathcal{X}) v_{u}^{\tau}\right\rangle$; using essentially the pseudolocal property of a pseudodifferential operator, we can show that this is rapidly decreasing.

We will use, then, a function $\mathscr{X} \in \mathscr{D}\left(\mathbf{R}^{n}\right)$ which is identically 1 in a neighborhood of the origin, and we write

$$
\left\langle\tau^{-N} g_{\xi}^{\tau}, \tau^{-m_{v}} v_{u}^{\tau}\right\rangle=\left\langle\tau^{-N} g_{\xi}^{\tau}, \tau^{-m} \mathcal{X} v_{u}^{\tau}\right\rangle+\left\langle g, \tau^{-N-m_{u}}\right\rangle
$$

where

$$
\begin{aligned}
r_{u}^{\tau}(x)= & {[1-\mathcal{X}(\sqrt{\tau} x)] \tau^{n / 2} } \\
& \cdot(2 \pi)^{-n} \int e^{i\langle\sqrt{\tau} \eta-\tau \xi, x\rangle} q(x, \sqrt{\tau} \eta-\tau \xi) \hat{u}(\eta) d \eta .
\end{aligned}
$$

Now the statement $O_{(0, \xi)}(P g) \leqslant N+m$ will be established once we have proven Lemmas 2.1.3 and 2.1.4 below. In fact, to show that the left-hand side of (2.1.6) is bounded for $\tau \geqslant 1$ and $u$ in a given bounded subset $A \subseteq \mathscr{D}\left(\mathbf{R}^{n}\right)$, we choose a function $\mathscr{X} \in \mathscr{D}\left(\mathbf{R}^{n}\right)$ which is identically 1 on a neighborhood of a compact set containing the supports of all $u \in A$, and we show that each term on the right-hand side of (2.1.6) remains bounded. For the first term, we use Lemma 2.1.3 together with the assumption that $\tau^{-N} g_{\xi}$ is a bounded family of distributions. For the second term, we choose $\Phi \in \mathscr{D}\left(\mathbf{R}^{n}\right)$ which is identically 1 on the support of $g$, so that $\left\langle g, \tau^{-N-m} r_{u}^{\tau}\right\rangle=\left\langle g, \tau^{-N-m} \Phi r_{u}^{\tau}\right\rangle$, 
and we apply Lemma 2.1 .4 with $l=-N-m$.

LEMMA 2.1.3. Let $A$ be a bounded subset of $\mathscr{D}\left(\mathbf{R}^{n}\right), \mathfrak{X} \in \mathscr{D}\left(\mathbf{R}^{n}\right)$. Then the set

$$
B=\left\{\tau^{-m} \mathcal{X} v_{u}^{\tau} \mid u \in A, \tau \geqslant 1\right\}
$$

is bounded in $\mathscr{D}\left(\mathbf{R}^{n}\right)$.

LEMMA 2.1.4. Suppose that $\mathcal{X}$ is identically 1 on a neighborhood of a compact set containing the supports of all $u$ in the bounded set $A$, and that $\Phi \in \mathscr{D}\left(\mathbf{R}^{n}\right)$. Then the set $\left\{\tau^{\prime} \Phi r_{u}^{\tau} \mid u \in A, \tau \geqslant 1\right\}$ is bounded in $\mathscr{D}\left(\mathbf{R}^{n}\right)$. ( $r_{u}^{\tau}$ is defined by (2.1.7).)

Proof of Lemma 2.1.3. It will suffice to show that $B$ is $C^{0}$ bounded. In fact, if we apply a differentiation $\partial / \partial x_{j}$ to a typical element

$$
w_{u}^{\tau}(x)=\tau^{-m} \mathcal{X}(x)(2 \pi)^{-n} \int e^{i\langle\eta, x\rangle} q\left(\frac{x}{\sqrt{\tau}}, \sqrt{\tau} \eta-\tau \xi\right) \hat{u}(\eta) d \eta
$$

of $B$, the result is a sum of expressions of the same form with $\mathcal{X}$ replaced by $\partial \mathcal{X} / \partial x_{j}, u$ replaced by $\partial u / \partial x_{j}$ (if $e^{i\langle\eta, x\rangle}$ is differentiated), or $q$ replaced by $\tau^{-1 / 2} \partial q / \partial x_{i}$; the latter has the same $\tau$-order as $q$ because $\delta \leqslant 1 / 2$.

To simplify notation we will denote by the letter $C$ any constant which can be chosen uniformly for all $u \in A$ and all $\tau \geqslant 1$.

The convex hull of the support of $\mathcal{X}$ and the origin is compact, and $q \in S_{\rho, \delta}^{m}$, so we have

$$
\mathcal{X}(x)|q(x / \sqrt{\tau}, \sqrt{\tau} \eta-\tau \xi)|<C(1+|\sqrt{\tau} \eta-\tau \xi|)^{m} .
$$

Since $A$ is bounded in $\mathscr{D}\left(\mathbf{R}^{n}\right)$, it is also bounded in $\mathcal{S}\left(\mathbf{R}^{n}\right)$, so the $\hat{u}$ 's are uniformly rapidly decreasing, and we have

$$
|\hat{u}(\eta)| \leqslant C(1+|\eta|)^{-j}
$$

for each $j$. (Here $C$ depends on $j$.)

Applying (2.1.9) and (2.1.10) to (2.1.8) gives

$$
\left|w_{u}^{\tau}(x)\right| \leqslant C \tau^{-m} \int(1+|\sqrt{\tau} \eta-\tau \xi|)^{m}(1+|\eta|)^{-j} d \eta
$$

or

$$
\left|w_{u}^{\tau}(x)\right|<C \int\left(\frac{1}{\tau}+\left|\frac{\eta}{\sqrt{\tau}}-\xi\right|\right)^{m}(1+|\eta|)^{-j} d \eta .
$$

If $m \geqslant 0$, we can replace this by

$$
\left|w_{u}^{\tau}(x)\right| \leqslant C \int(1+|\eta|+|\xi|)^{m}(1+|\eta|)^{-j} d \eta .
$$

For $j$ sufficiently large, this integral converges, and we have $\left|w_{u}^{\tau}(x)\right| \leqslant C$. 
If $m<0$, we have a little more work to do because the integrand in (2.1.11) becomes large as $\tau \rightarrow \infty$ for $\eta=\xi \sqrt{\tau}$. We write the integral in (2.1.11) as $I_{1}+I_{2}$, where $I_{1}$, is taken over $\left\{\eta \| \eta-\sqrt{\tau} \xi\left|\geqslant \frac{1}{2} \sqrt{\tau}\right| \xi \mid\right\}$ and $I_{2}$ is taken over $\left\{\eta|| \eta-\sqrt{\tau} \xi\left|\leqslant \frac{1}{2} \sqrt{\tau}\right| \xi \mid\right\}$. Since $m<0$, we have

$$
I_{1} \leqslant C \int\left(\frac{1}{\tau}+|\xi|\right)^{m}(1+|\eta|)^{-j} d \eta \leqslant 2 C|\xi|^{m} \int(1+|\eta|)^{-j} d \eta
$$

which is finite if $j$ is sufficiently large. The domain of integration in $I_{2}$ has volume $C \tau^{n / 2}$, and the factors $(1 / \tau+|\eta / \sqrt{\tau}-\xi|)^{m}$ and $(1+|\eta|)^{-j}$ in the integrand are bounded respectively by $\tau^{-m}$ and $\left(1+\frac{1}{2} \sqrt{\tau}|\xi|\right)^{-j}$, so we have

$$
I_{2} \leqslant C \tau^{-m+n / 2}\left(1+\frac{1}{2} \sqrt{\tau}|\xi|\right)^{-j} .
$$

Choosing $j \geqslant-2 m+n$, we see that $I_{2}$ is bounded. (We remark for later use that $I_{2}$ is actually rapidly decreasing.)

Proof of Lemma 2.1.4. We can reduce the problem to one of proving $C^{0}$ boundedness, as in Lemma 2.1.3, if we replace the function $1-\mathcal{X}(x)$ by any function $\Gamma(x)$ which vanishes on a neighborhood of a compact set containing the supports of all $u \in A$ and which is bounded, together with each of its partial derivatives, on all of $\mathbf{R}^{n}$. Thus, we are trying to prove that, as $u$ ranges over $A$ and $\tau$ over $[1, \infty)$, the functions

$$
\tilde{S}_{u}^{\tau}(x)=\tau^{\prime} \Phi(x) \Gamma(\sqrt{\tau} x) \int e^{i\langle\sqrt{\tau} \eta-\tau \xi, x\rangle} q(x, \sqrt{\tau} \eta-\tau \xi) \hat{u}(\eta) d \eta
$$

form a bounded set in $\mathscr{D}\left(\mathbf{R}^{n}\right)$. (Note that, in passing from (2.1.7) to (2.1.12) we have ignored the constant factor $(2 \pi)^{-n}$ and have absorbed the $\tau^{n / 2}$ into $\tau^{l}$, since $l$ is to be arbitrary.)

To show that it suffices to prove $C^{0}$ boundedness, we note that $\partial \tilde{s}_{u}^{\tau} / \partial x_{j}$ is a sum of terms of the same form as $\tilde{s}_{u}^{\tau}(x)$, with $\Phi$ replaced by $\partial \Phi / \partial x_{j}, \Gamma$ replaced by $\partial \Gamma / \partial x_{j}$ and $l$ replaced by $l+1 / 2$, or $q(x, \eta)$ replaced by $i \eta q(x, \eta)$ (which belongs to $S_{\rho, \delta}^{m+1}$ ). The problem is reduced, then, to finding an estimate for

$$
s_{u}^{\tau}(x)=\tau^{\prime} \Gamma(\sqrt{\tau} x) \int e^{i\langle\sqrt{\tau} \eta-\tau \xi, x\rangle} q(x, \sqrt{\tau} \eta-\tau \xi) \hat{u}(\eta) d \eta
$$

which is uniform for $\tau \geqslant 1, u \in A$, and $x$ in a compact ball $B$ containing the support of $\Phi$.

If $l$ happens to be less than or equal to $-m$ we can write

$$
\left|s_{u}^{\tau}(x)\right| \leqslant \tau^{-m} \Gamma(\sqrt{\tau} x) \int e^{i\langle\sqrt{\tau} \eta-\tau \xi, x\rangle} q(x, \sqrt{\tau} \eta-\tau \xi) \hat{u}(\eta) d \eta
$$

and use the method of proof in Lemma 2.1.3 to show that the right-hand side is bounded. The factor $\Gamma(\sqrt{\tau} x)$ poses no problem because $\Gamma$ is bounded on $\mathbf{R}^{n}$. 
For general $l$, we must reduce to the case $l \leqslant-m$ by integrating by parts with respect to $\eta$ in order to lower the order of growth of $q$. This requires special care because $e^{i\langle\sqrt{\tau} \eta-\tau \xi, x\rangle} \hat{u}(\eta)$ rather than just $e^{i\langle\sqrt{\tau} \eta-\tau \xi, x\rangle}$ must be antidifferentiated. We will use the laplacian

$$
\Delta_{\eta}=-\left(\partial^{2} / \partial \eta_{1}^{2}+\cdots+\partial^{2} / \partial \eta_{n}^{2}\right)
$$

raised to the $k$ th power, where $k$ is to be chosen later. Solving the equation

$$
\Delta_{\eta}^{k} \varphi=e^{i\langle\eta, z\rangle} \hat{u}(\eta)
$$

by Fourier transforms, we find that $e^{i\langle\eta, z\rangle} \hat{u}(\eta)=\Delta_{\eta}^{k}\left[e^{i\langle\eta, z\rangle} \hat{b}_{z, u}(\eta)\right]$ where

$$
b_{z, u}(y)=|y-z|^{-2 k} u(y) \text {. }
$$

(We will use these formulas only when $z$ lies outside the support of $u$.) It follows that

$$
s_{u}^{\tau}(x)=\tau^{l} \Gamma(\sqrt{\tau} x) \int \Delta_{\eta}^{k}\left(e^{i\langle\sqrt{\tau} \eta-\tau \xi, x\rangle} \widehat{b \sqrt{\tau} x, u}(\eta)\right) q(x, \sqrt{\tau} \eta-\tau \xi) d \eta .
$$

Now we can use the selfadjointness of $\Delta_{\eta}^{k}$, even though the integrand is not compactly supported. In fact, if $\alpha(\eta)$ is rapidly decreasing and $\beta(\eta)$ is slowly growing, the equality $\int \Delta \alpha \cdot \beta d \eta=\int \alpha \cdot \Delta \beta d \eta$ is true because the "boundary term" is rapidly decreasing. Thus we have

$$
\begin{aligned}
s_{u}^{\tau}(x)=\tau^{l+k} \Gamma(\sqrt{\tau} x) \int e^{i\langle\sqrt{\tau} \eta-\tau \xi, x\rangle} \widehat{b_{\sqrt{\tau} x, u}}(\eta) & \cdot\left(\Delta_{\eta}^{k} q\right)(x, \sqrt{\tau} \eta-\tau \xi) d \eta .
\end{aligned}
$$

Now (2.1.15) is an expression of the same form as (2.1.13), with $l$ replaced by $l+k / 2, q$ replaced by $\Delta_{\eta}^{k} q$, and $u$ replaced by $b_{\sqrt{\tau} x, u}$. Since $\Delta_{\eta}^{k} q$ belongs to $S_{\rho, \delta}^{m-2 k \rho}$, we can apply the case " $l \leqslant-m$ " if we know two things:

(i) $l+k \leqslant-m+2 k \rho$;

(ii) $\left\{b_{\sqrt{\tau} x, u} \mid \sqrt{\tau} \geqslant 1, u \in A, \Gamma(\sqrt{\tau} x) \neq 0\right\}$ is bounded in $\mathscr{D}\left(\mathbf{R}^{n}\right)$.

We can make (i) true simply by choosing $k$ large enough, since $\rho>1 / 2$. All that remains, then, is to establish (ii). From (2.1.14), we have

$$
b_{\sqrt{\tau} x, u}(y)=|y-\sqrt{\tau} x|^{-2 k} u(y)
$$

Let $\delta>0$ be the distance between the support of $\Gamma$ and the union of the supports of all $u \in A$. Then we have the estimate $\left|b_{\sqrt{\tau} x, u}(y)\right| \leqslant \delta^{-k}|u(y)|$, so the $b_{\sqrt{\tau} x, u}$ 's are supported in a fixed compact set and are $C^{0}$ bounded there. Differentiating (2.1.16) gives similar estimates for the derivatives of $b \sqrt{\tau} x, u$, with $\delta^{-k}$ replaced by $\delta^{-k-j}$ if $j$ derivatives are taken, so (ii) is established, and our proof is complete.

We come now to the task of analyzing the symbol $\sigma_{(0, \xi)}^{N+m}(P g)$. Our first step will be to prove part (b) of the theorem, i.e., that this symbol depends only on the behavior of $q$ in a conic neighborhood of $(0,-\xi)$. We consider, then, a 
symbol $\tilde{q}(x, \eta) \in S_{\rho, \delta}^{m}$ with the following property:

$$
\left\{\begin{array}{l}
\text { there is a neighborhood } \mathcal{U} \text { of } u \text { in } \mathbf{R}^{n}, \\
\text { a conic neighborhood } \Gamma \text { of }-\xi \text { in } \mathbf{R}^{n *}, \\
\text { and a constant } K>0 \text { such that } \\
\tilde{q}(x, \eta)=0 \text { whenever } x \in \mathcal{Q}, \eta \in \Gamma \text {, and }|\eta|>K .
\end{array}\right\}
$$

We will show that, if $A$ is a bounded subset of $\mathscr{D}\left(\mathbf{R}^{n}\right)$, then the family $\left\langle g_{\xi}^{\tau}, \tilde{v}_{u}^{\tau}\right\rangle$, where $\tilde{v}_{u}^{\tau}$ is defined by (2.1.5) with $q$ replaced by $\tilde{q}$, is rapidly decreasing, uniformly for $u \in A$. The formula (2.1.6), together with Lemma 2.1.4, shows that, if we introduce a cutoff function $\mathcal{X} \in \mathscr{D}\left(\mathbf{R}^{n}\right)$ which is identically 1 on a neighborhood of a compact set containing the supports of all $u \in A$, then it suffices to show that $\left\langle g_{\xi}^{\tau},\left\{\tilde{v}_{u}^{\tau}\right\rangle\right.$ is rapidly decreasing. Since $g_{\xi}^{\tau}$ has polynomial growth in $\tau$, the problem comes down to showing that $\mathcal{X} \tilde{v}_{u}^{\tau}$ is rapidly decreasing.

Now we may choose $\varepsilon>0$ such that an $\varepsilon$-neighborhood of $-\xi$ lies in $\Gamma$ and avoids the origin. For $|\eta|<\varepsilon \sqrt{\tau}$, therefore, $\eta / \sqrt{\tau}-\xi$ lies in $\Gamma$; for $\tau$ sufficiently large, $\sqrt{\tau} \eta-\tau \xi$ lies in $\Gamma$, and $|\sqrt{\tau} \eta-\tau \xi|>K$. Looking at the $x$-variables, we find that for $\tau$ sufficiently large and any $x$, either $x / \sqrt{\tau}$ belongs to $\mathcal{Q}$ or $\mathcal{X}(x)=0$. It follows from (2.1.17) that the integral for $|\eta|<\varepsilon \sqrt{\tau}$ makes no contribution to $\mathscr{X} \tilde{v}_{u}^{\tau}$, so it suffices to prove the following lemma.

LEMMA 2.1.5. Let $\mathcal{X} \in \mathscr{D}\left(\mathbf{R}^{n}\right), q(x, \xi) \in S_{\rho, \delta}^{m}\left(\mathbf{R}^{n} \times \mathbf{R}^{n *}\right), \varepsilon>0$, and $A \subseteq$ $\mathscr{D}\left(\mathbf{R}^{n}\right)$ a bounded subset. Then the family $\mathscr{X}(x) \tilde{v}_{u}^{\tau}$ is rapidly decreasing in $\mathscr{D}\left(\mathbf{R}^{n}\right)$ uniformly for $u \in A$, where

$$
\hat{v}_{u}^{\tau}(x)=\int_{|\eta|>\sqrt{\tau} \varepsilon} e^{i\langle\eta, x\rangle} q\left(\frac{x}{\sqrt{\tau}}, \sqrt{\tau} \eta-\tau \xi\right) \hat{u}(\eta) d \eta .
$$

Proof. As in the preceding two lemmas it suffices to show that, for any integer $l, \tau^{l}\left|\hat{v}_{u}^{\tau}(x)\right|$ is uniformly bounded for $x$ in the support of $\mathcal{X}$. We use the estimate

If $m \leqslant 0$ we simply have

$$
\left|\hat{v}_{u}^{\tau}(x)\right| \leqslant C \int_{|\eta|>\sqrt{\tau} \varepsilon}(1+|\sqrt{\tau} \eta-\tau \xi|)^{m}|\hat{u}(\eta)| d \eta .
$$

$$
\left|\hat{v}_{u}^{\tau}(x)\right| \leqslant C \int_{|\eta|>\sqrt{\tau} \varepsilon}|\hat{u}(\eta)| d \eta,
$$

which is uniformly rapidly decreasing because $\hat{u}(\eta)$ is. If $m \geqslant 0$ we have, for $|\tau| \geqslant 1$,

$$
\begin{aligned}
(1+|\sqrt{\tau} \eta-\tau \xi|)^{m} & =\tau^{m}\left(\frac{1}{\tau}+\left|\frac{\eta}{\sqrt{\tau}}\right|+|\xi|\right)^{m} \\
& \leqslant \tau^{m}(1+|\xi|+|\eta|)^{m} \leqslant C \tau^{m}(1+|\eta|)^{m}
\end{aligned}
$$


so

$$
\left|\hat{v}_{u}^{\tau}(x)\right| \leqslant C \tau^{m} \int_{|\eta|>\tau \varepsilon}(1+|\eta|)^{m}|\hat{u}(\eta)| d \eta,
$$

which is again rapidly decreasing.

Suppose now that $P$ is altered by an operator whose total symbol vanishes on a conic neighborhood of $(0, \xi)$. By formula (2.1.6), $P^{t}$ is altered by an operator whose total symbol $\tilde{q}$ vanishes on a conic neighborhood of $(0,-\xi)$, plus an operator whose total symbol $\tilde{\tilde{q}}$ lies in $S_{\rho, \delta}^{-\infty}$. By Lemma 2.1 .5 and the discussion preceding it, the operator corresponding to $\tilde{q}$ has no effect on $\sigma_{(0, \xi)}^{M+n}(g)$; the second operator is a smoothing operator and, likewise, has no effect on the symbol.

Suppose, finally, that $P$ has a principal symbol of type $(0,1)$. Applying our formula about orders, for an operator of order $m-1 / 2$, we assume that $P$ itself has a total symbol of type $(0,1)$ and proceed to establish the boundedness of the family

$$
\Delta^{\tau}=\tau^{-N-m+1 / 2}\left((P g)_{\xi}^{\tau}-q(0,-\tau \xi) g_{\xi}^{\tau}\right) .
$$

If $u$ is any test function, Fourier's inversion formula and some transformations like ones we have done before yield

$$
\begin{aligned}
&\left\langle\Delta^{\tau}, u\right\rangle=\left\langle\tau^{-N} g_{\xi}^{\tau}, \tau^{-m+1 / 2}(2 \pi)^{-n}\right. \\
&\left.\cdot \int e^{i\langle\eta, x\rangle}\right\rangle\left[q\left(\frac{x}{\sqrt{\tau}}, \sqrt{\tau} \eta-\tau \xi\right)-q(0,-\tau \xi)\right] \hat{u}(\eta) d \eta .
\end{aligned}
$$

By Lemmas 2.1.4 and 2.1.5, we will have ignored only rapidly decreasing terms if we replace this family by

$$
\left\langle\tau^{-N} g_{\xi}^{\tau},(2 \pi)^{-n} \tau^{-m+1 / 2} \bar{v}_{u}^{\tau}\right\rangle,
$$

where

$$
\bar{v}_{u}^{\tau}(x)=\mathcal{X}(x) \int_{|\eta|<\varepsilon \sqrt{\tau}} e^{i\langle\eta, x\rangle}\left[q\left(\frac{x}{\sqrt{\tau}}, \sqrt{\tau} \eta-\tau \xi\right)-q(0,-\tau \xi)\right] \hat{u}(\eta) d \eta,
$$

$\mathcal{X} \in \mathscr{D}\left(\mathbf{R}^{n}\right)$ being identically 1 near the origin and $0<\varepsilon$. Once again, we are left with the problem of showing that $\left\{\tau^{-m+1 / 2} \bar{v}_{u}^{\tau} \mid \tau>1, u \in A\right\}$ is bounded; as usual, it suffices to check $C^{0}$ boundedness because each derivative is a sum of terms of the same form. (Or of simpler terms: If we apply $\partial^{|\alpha|} / \partial x^{\alpha}$ to $[q(x / \sqrt{\tau}, \sqrt{\tau} \eta-\tau \xi)-q(0,-\tau \xi)]$, we get

$$
\tau^{-|\alpha| / 2} \frac{\partial^{|\alpha|} q}{\partial x^{\alpha}}\left(\frac{x}{\sqrt{\tau}}, \sqrt{\tau} \eta-\tau \xi\right) .
$$

$\partial^{|\alpha|} q / \partial x^{\alpha}$ still belongs to $S_{\rho, \delta}^{m}$ since $\delta=0$, and we have gained a $\tau^{-1 / 2}$, so we can use Lemma 2.1.3.) 
To estimate $\tau^{-m+1 / 2}\left|\bar{v}_{u}^{\tau}(x)\right|$ on the support of $\mathcal{X}$, we use the mean value theorem to estimate

$$
\Delta=|q(x / \sqrt{\tau}, \sqrt{\tau} \eta-\tau \xi)-q(0,-\tau \xi)| .
$$

Let $M_{t}$ be the maximum value of $\Sigma\left|\left(\partial q / \partial x_{j}\right)(x, \eta)\right|$ for $x \in \operatorname{Supp} \mathcal{X},|\eta| \leqslant t$; $M_{t} \leqslant C(1+t)^{m}$ because $\delta=0$. Let $N_{t}$ be the maximum value of $\Sigma\left|\left(\partial q / \partial \xi_{j}\right)(x, \eta)\right|$ for $x \in \operatorname{Supp} \mathcal{X},|\eta| \leqslant \tau ; N_{t} \leqslant C(1+t)^{m-1}$ because $\rho=$ 1. The mean value theorem implies that $\Delta \leqslant M_{t}|x / \sqrt{\tau}|+N_{t}|\sqrt{\tau} \eta|$ for $t=|\sqrt{\tau} \eta|+|\tau \xi|$, so we have

$$
\Delta \leqslant C\left[(1+\sqrt{\tau}|\eta|+\tau|\xi|)^{m}|x| / \sqrt{\tau}+(1+\sqrt{\tau}|\eta|+\tau|\xi|)^{m-1} \sqrt{\tau}|\eta|\right] .
$$

Since the integral is taken over $|\eta| \leqslant \varepsilon \sqrt{\tau}$, we have

$$
\Delta \leqslant C\left[(1+(\varepsilon+|\xi|) \tau)^{m}|x| / \sqrt{\tau}+(1+(\varepsilon+|\xi|) \tau)^{m-1} \sqrt{\tau}|\eta|\right] .
$$

This implies

$$
\begin{aligned}
\tau^{-m+1 / 2} \Delta & \leqslant C\left[(1 / \tau+(\varepsilon+|\xi|))^{m}|x|+(1 / \tau+(\varepsilon+|\xi|))^{m}|\eta|\right] \\
& \leqslant C[1 / \tau+(\varepsilon+|\xi|)]^{m}[1+|\eta|] .
\end{aligned}
$$

(We incorporate $|x|$ into $C$ in the last step.) On the support of $\mathcal{X}$, therefore, we have

$$
\begin{aligned}
\tau^{-m+1 / 2}\left|\bar{v}_{u}^{\tau}(x)\right| & \leqslant C \int_{\eta<\varepsilon \sqrt{\tau}}\left[\frac{1}{\tau}+(\varepsilon+|\xi|)\right]^{m}[1+|\eta|]|\hat{u}(\eta)| d \eta \\
& \leqslant C \int_{\mathbf{R}^{n}}\left[\frac{1}{\tau}+(\varepsilon+|\xi|)\right]^{m}[1+|\eta|]|\hat{u}(\eta)| d \eta .
\end{aligned}
$$

At this point we can repeat the argument used at the end of the proof of Lemma 2.1.3, breaking the integral into two parts if $m<0$, to conclude that $\tau^{-m+1 / 2}\left|\bar{v}_{u}^{\tau}(x)\right|$ is bounded.

Since we have shown that $\tau^{-N-m+1 / 2}\left((P g)_{\xi}^{\tau}-q(0, \tau \xi) g_{\xi}^{\tau}\right)$ is bounded, and we know that $q(0,-\tau \xi)=p(0, \tau \xi)$, we conclude that

$$
(P g)_{\xi}^{\tau}-p(0, \xi) g_{\xi}^{\tau}=O\left(\tau^{N+m-1 / 2}\right) \text {, }
$$

and the proof of Theorem 2.1.2(c) is complete. Part (d) follows from (c) and Definition 1.3.12.

REMARK 2.1.6. L. Hörmander has pointed out that our proof of Theorem 2.1.2 can be simplified by the use of the estimate in Theorem 2.6 of [5a]. (This is a "Taylor's formula with remainder" for pseudodifferential operators.) $\mathrm{K}$. Sklower has carried out this simplification and has been able to extend Theorem 2.1.2 to obtain formulas of "transport-equation" type for lower order symbols of $P g$ at points where the principal symbol of $P$ and some of its derivatives vanish. Details will appear in Sklower's Berkeley Ph.D. thesis. 
2.2. The symbol and the wavefront set. A direct consequence of Theorem 2.1.2 is the following "regularity theorem".

COROLlaRY 2.2.1. Let $P$ be a properly supported pseudodifferential operator of order $m$ and type $(1,0)$ which is elliptic at $(0, \xi)$ in the sense that $\tau^{-m}(p(0, \tau \xi))$ is bounded away from 0 for large $\tau$. Then

$$
O_{(0, \xi)}(P g) \leqslant N+m \Rightarrow O_{(0, \xi)}(g) \leqslant N .
$$

Proof. Suppose $O_{(0, \xi)}(g)=M>N$. Then $\sigma_{(0, \xi)}^{M}(g) \neq 0$; i.e. $\tau^{-M+1 / 2} g_{\xi}^{\tau}$ is unbounded. By the ellipticity of $P, \tau^{-M-m+m / 2} p(0, \tau \xi) g_{\xi}^{\tau}$ is unbounded as well. By Theorem 2.1.2

$$
p(O, \tau \xi) g_{\xi}^{\tau} \equiv(P g)_{\xi}^{\tau}\left(\bmod O\left(\tau^{M+m-1 / 2}\right)\right),
$$

so $\tau^{-M-m+1 / 2}(P g)_{\xi}^{\tau}$ is unbounded; i.e. $\sigma_{(0, \xi)}^{M+m}(P g) \neq 0$, and $O_{(0, \xi)}(P g)=M+$ $m>N+m$, contradicting the hypothesis on $P g$.

CoROllaRY 2.2.2. If $g \in \mathscr{Q}^{\prime}\left(\mathbf{R}^{n}\right)$ and $\xi \neq 0$, then $O_{(0, \xi)}(g)=-\infty$ if $(0, \xi)$ is not in the wavefront set of $g$.

Proof. By the definition on p. 120 of [6], if $(0, \xi)$ is not in the wavefront set of $g$, there is a properly supported pseudodifferential operator $P$ of order 0 and type $(1,0)$, elliptic at $(0, \xi)$, such that $P g$ is a $C^{\infty}$ function. By Proposition 1.2.5, $O_{(0, \xi)}(P g)=-\infty$; by Corollary 2.2.1, we have $O_{(0, \xi)}(g)=-\infty$.

ReMARK 2.2.3. Y. Colin de Verdière and L. Hörmander [private communications] have pointed out that Corollary 2.2.2 can be proven directly. In fact, if $(0, \xi) \notin \mathrm{WF}(g)$, we can cut off $g$, without changing its germ at the origin, so that $\hat{g}(\xi)$ is uniformly rapidly decreasing on a conic neighborhood $\Re$ of $\xi$. A formal computation shows that, for any test function $u$,

$$
\left\langle g_{\xi}^{\tau}, u\right\rangle=\int_{\mathbf{R}^{n}} \hat{g}(\tau \xi-\eta) \hat{u}\left(\frac{\eta}{\sqrt{\tau}}\right) d \eta .
$$

We must show that this is rapidly decreasing in $\tau$.

For $\varepsilon$ sufficiently small, and $|\eta| \leqslant \varepsilon \tau$, we have $\tau \xi-\eta \in \Re$ and $|\tau \xi-\eta| \geqslant$ $C \tau$ for some positive constant $C$. Now we break the integral in (2.2.1) into two parts, $\left\langle g_{\xi}^{\tau}, u\right\rangle=I_{1}(\tau)+I_{2}(\tau)$, where $I_{1}$ is taken over $|\eta| \leqslant \varepsilon \tau$ and $I_{2}$ over $|\eta|>\varepsilon \tau$. Since $\hat{g}$ is rapidly decreasing in $\Re$ and $u$ is bounded, $I_{1}(\tau)=$ $O\left(\tau^{-\infty}\right)$. On the other hand, in $I_{2}$, we have $\eta / \sqrt{\tau} \geqslant \varepsilon \sqrt{\tau}$, so that $\hat{u}(\eta / \sqrt{\tau})$ is rapidly decreasing while $\hat{g}(\tau \xi-\eta)$ has polynomial growth. It follows that $I_{2}(\tau)=O\left(\tau^{-\infty}\right)$ as well. All these estimates can be made uniform for $u$ in a bounded set of test functions, so we may conclude that $O_{(0, \xi)}(g)=-\infty$.

It is natural to ask whether the converse to Corollary 2.2.2 is true; the following example, due to Colin de Verdière [private communication] shows that it is false. 
EXAMPLE 2.2.4. Let $g \in \mathscr{D}^{\prime}\left(\mathbf{R}^{2}\right)$ be the distribution whose Fourier transform $\hat{g}$ is defined by

$$
\langle\hat{g}, v\rangle=\int_{0}^{\infty} v\left(t, t^{\beta}\right) d t
$$

where $\beta$ is a constant between $1 / 2$ and 1 . We shall show that $g$ is not $C^{\infty}$ at the origin, so that its wavefront set contains a point of the form $(x, \xi)$, with $\xi \neq 0$. On the other hand, we will show that $O_{(x, \xi)}(g)=-\infty$ for all $x$ and all $\xi \neq 0$. Thus, it is not even true that the set $S_{g}=\left\{(x, \xi) \mid \xi \neq 0\right.$ and $O_{(x, \xi)}(g)>$ $-\infty\}$ is dense in $\operatorname{WF}(g)$. (This is the best we could have hoped for, since $\mathrm{WF}(g)$ is always closed, while $S_{g}$ might not be.)

Suppose, then, that $g$ is $C^{\infty}$ in a neighborhood of 0 . Let $\Psi\left(x_{1}, x_{2}\right) \in \mathscr{D}\left(\mathbf{R}^{2}\right)$ be an even, nonnegative function, positive near 0 , and having support so small that $(\psi * \psi) g$ is a $C^{\infty}$ function. The Fourier transform $\hat{\varphi}=\overline{\psi * \psi}$ is then nonnegative and positive near $\xi=0$, while $\varphi g=\hat{\varphi} * \hat{g}$ is rapidly decreasing. But this is impossible because $\hat{g}$ is nonnegative and so its convolution with $\hat{\varphi}$ could not be rapidly decreasing along the curve $t \mapsto$ $\left(t, t^{\beta}\right)$ as $t \rightarrow \infty$.

To show that $O_{(x, \xi)}(g)<-\infty$ whenever $\xi \neq 0$, one first does a formal computation (left to the reader) which shows that it suffices to prove that, for any $u \in \mathscr{D}\left(\mathbf{R}^{2}\right)$, the integral

$$
I=\int_{\mathbf{R}}\left|\hat{u}\left(\sqrt{\tau} \xi_{1}-\frac{t}{\sqrt{\tau}}, \sqrt{\tau} \xi_{2}-\frac{t^{\beta}}{\sqrt{\tau}}\right)\right| d t
$$

is rapidly decreasing as $\tau \rightarrow \infty$ whenever $\left(\xi_{1}, \xi_{2}\right) \neq(0,0)$. Making the substitution $t \mapsto \sqrt{\tau} t$ and using the fact that $\hat{u}$ is rapidly decreasing, we obtain, for any $N$, an inequality of the form

$$
I \leqslant C \int_{\mathbf{R}} \frac{d t}{\left(1+\left|\sqrt{\tau} \xi_{1}-t\right|+\left|\sqrt{\tau} \xi_{2}-t^{\beta} / \tau^{(1-\beta) / 2}\right|\right)} .
$$

Now one chooses a number $\delta$ between 0 and $1 / 2$ and writes the integral on the right-hand side of (2.2.2) as a sum $I_{1}+I_{2}$, where $I_{1}$ is the integral for $\left|t-\sqrt{\tau} \xi_{1}\right|<\tau^{\delta}$ and $I_{2}$ is the integral for $\left|t-\sqrt{\tau} \xi_{1}\right|>\tau^{\delta}$. Then one can show that

$$
I_{1}=\left\{\begin{array}{ll}
O\left(\tau^{\delta-N / 2}\right) & \text { if } \xi_{2} \neq 0, \\
O\left(\tau^{\delta-N(\beta-1 / 2)}\right) & \text { if } \xi_{2}=0 \text { and } \xi_{1} \neq 0
\end{array}\right\}
$$

and $I_{2}=O\left(\tau^{\delta / N-\delta N}\right)$. It follows, since $N$ is arbitrary, that $I_{1}$ and $I_{2}$ are rapidly decreasing, so $I$ is rapidly decreasing as well. 


\section{The Symbol of a Fourier INTEgRal Distribution}

3.0. Introduction. Hörmander [6] defined, for any conic lagrangian submanifold $\Lambda \subseteq T^{*} X$ and any real number $m$, a class $I^{m}(X, \Lambda)$ of distributions on $X$, with wavefront set in $\Lambda$, called Fourier integral distributions. For each $h \in I^{m}(X, \Lambda)$ he constructed a principal symbol which is a section of a certain complex line bundle over $\Lambda$.

Our starting point for the work described in this paper was a universal construction of Hörmander's sumbol bundles in terms of the twisted distribution spaces defined in $\S 1.3$. This suggested the idea that one might be able to define Hörmander's symbol directly by means of a localization process which passed from distributions on $X$ to twisted distributions on the tangent spaces of $X$. After some experimentation, we found the localization process described in $\$ 1.1$.

In this chapter, we show that our localization process does indeed give Hörmander's principal symbol. We prove in $\$ 3.2$ that, if $h \in I^{m}(X, \Lambda)$ with homogeneous principal symbol in Hörmander's sense, then, at each $\xi \in \Lambda, g$ is homogeneous in our sense (see Definition 1.3.12 and Remark 1.6.4) of order $m+n / 4$, and we compute the symbol in a special coordinate system. In \$3.3, we show that these symbols belong to a special 1-dimensional subspace of $\operatorname{DD}^{\prime}\left(T_{x} X\right)$ which depends only upon $T_{\xi} \Lambda$. Finally, we show that the collection of these 1-dimensional subspaces for all $\xi \in \Lambda$ can be identified with Hörmander's symbol bundle $\mathfrak{T}_{\Lambda}$, and we show that our symbol and Hörmander's are equal. In case the principal symbol is not homogeneous, our symbol at $\xi$, as a function of $\tau$, is equal to Hörmander's symbol at $\tau \xi$.

To compute our symbol we use, as did Hörmander, the principle of stationary phase, but our calculations require some refinements of this principle, since the presence of the homotheties in the $x$-variables prevents us from applying the Morse lemma to the phase function. These refinements are discussed in $\$ 3.1$.

3.1. On the principle of stationary phase. The principle of stationary phase in its basic form is an asymptotic expansion as $t \rightarrow \infty$ for the integral

$$
I(t)=\int e^{i t Q(x)} u(x) d x
$$

where $Q(x)$ is a nondegenerate quadratic form on $\mathbf{R}^{k}$ and $g(x)$ is a compactly supported $C^{\infty}$ function. We begin this section by reviewing the derivation of this principle, following [2] and [6].

Writing $Q(x)=\frac{1}{2}\langle A x, x\rangle$, where $A$ is an invertible symmetric matrix, we define $\tilde{Q}$ by $\tilde{Q}(\xi)=\frac{1}{2}\left\langle A^{-1} \xi, \xi\right\rangle$. Applying the Fourier transform inside the integral in (3.1.1), one obtains

$$
I(t)=(2 \pi t)^{-k / 2}|\operatorname{det} A|^{-1 / 2} e^{(i \pi / 4) \operatorname{sgn} A} J(t),
$$


where

$$
J(t)=\int e^{-i \tilde{Q}(\xi) / t} \hat{u}(\xi) d \xi .
$$

Using the finite Taylor expansion with remainder estimate

$$
\left|e^{i s}-\sum_{j=0}^{\nu-1} \frac{(i s)^{j}}{j !}\right| \leqslant \frac{|s|^{\nu}}{\nu !}
$$

we obtain from (3.1.3) the inequality

$$
\begin{gathered}
\left|J(t)-\sum_{j=0}^{\nu-1} \int \frac{1}{j !}\left[\frac{-i \tilde{Q}(\xi)}{t}\right]^{j} \hat{u}(\xi) d \xi\right| \\
\leqslant \frac{1}{t^{\nu} \nu !} \int\left|\tilde{Q}(\xi)^{\nu} \hat{u}(\xi)\right| d \xi .
\end{gathered}
$$

Denoting by $\tilde{Q}(D)$ the second order differential operator obtained by substituting $i \partial / \partial x_{j}$ for $\xi_{j}$ in $\tilde{Q}(\xi)$, we obtain from (3.1.4)

$$
\left|J(t)-\sum_{j=0}^{\nu-1}\left\{\frac{1}{j !}\left[\frac{-i \tilde{Q}(D)}{t}\right]^{j} u\right\}(0)\right| \leqslant \frac{1}{\nu ! t^{\nu}} \int \widehat{\mid \tilde{Q}(D)^{\nu}} u(\xi) \mid d \xi .
$$

To estimate the right-hand side of (3.1.5) in terms of $u$ itself, we use the fact that for any $v$ and $n$,

$$
|\hat{v}(\xi)| \leqslant \frac{1}{\left(1+|\xi|^{2}\right)^{n / 2}} \int\left|\left\{(1+\Delta)^{n / 2} v\right\}(x)\right| d x,
$$

where $\Delta$ is the laplacian

$$
-\left(\partial^{2} / \partial x_{1}^{2}+\cdots+\partial^{2} / \partial x_{k}^{2}\right)=|D|^{2} .
$$

We denote by $\|v\|_{m}^{1}$ the maximum of the $L^{1}$ norms of all the partial derivatives of $v$ or order $\leqslant m$. Then, for $n$ even, the integral on the right-hand side of (3.1.6) is bounded above by $A_{n}\|v\|_{n}^{1}$, where $A_{n}$ is a constant which depends only on $n$ (and, of course, $k$ ). If $n$ is greater than $k$, the right-hand side of (3.1.6) is integrable with respect to $\xi$, and we have

$$
\int|\hat{v}(\xi)| d \xi \leqslant B_{n}\|v\|_{n}^{1} \quad(n>k)
$$

Combining (3.1.7) and (3.1.5) and writing $S_{\nu}(t)$ for the sum on the left-hand side of (3.1.5), we have

$$
\left|J(t)-S_{\nu}(t)\right| \leqslant C_{\nu} t^{-\nu}\left\|\tilde{Q}(D)^{\nu} u\right\|_{\tilde{k}}^{1}
$$

where $\bar{k}$ is the smallest even integer greater than $k$ and $C_{\nu}$ is a constant independent of $u$. The right-hand side of (3.1.8) can always be estimated by a 
constant times $t^{-\nu}\|u\|_{\bar{k}+2 v}^{1}$ (this is done, for example, in [6]) and this estimate is hard to improve if the quadratic form $Q$ is definite. If $\tilde{Q}$ is indefinite, however, then the operator $\tilde{Q}(D)^{\nu}$ is not elliptic, and the estimate in the form (3.1.8) can be a real advantage.

If we have an integral of the form $\int e^{i f f(x)} g(x) d x$, where $f$ has only nondegenerate critical points in the support of $g$, the usual approach is to use the Morse lemma to find local coordinates in which $f$ becomes quadratic. In the next section, however, the presence of the homotheties involved in the definition of the principal symbol will not allow us to make arbitrary coordinate changes, so we must renounce the use of the Morse lemma. We will sketch now an approach to the principle of stationary phase in which the Morse lemma is not used, leaving out the details because we will treat a similar but more complicated situation in the next section.

Suppose we wish to find an asymptotic expansion for the integral $I(t)=$ $\int e^{i f f(x)} g(x) d x$, where we assume that $f(x)$ has a nondegenerate critical point at the origin and no other critical points in the support of $g$. We may write $f(x)=Q(x)+R(x)$, where $Q(x)$ is a nondegenerate quadratic form and $R(x)$ vanishes to third order at the origin. Now we write

$$
I(t)=\int e^{i t Q(x)} e^{i t R(x)} g(x) d x
$$

and think of $e^{i t R(x)} g(x)$ as the $u(x)$ in (3.1.1). Since $e^{i t R(x)} g(x)$ now depends upon $t$ as well as $x$, in order to have a good asymptotic expansion for $I(t)$ we must show that the error terms $\left|J(t)-S_{\nu}(t)\right|$ are bounded by powers of $t$ which go to $-\infty$ as $\nu \rightarrow \infty$. Attempting to use the estimate

$$
\left|J(t)-S_{\nu}(t)\right| \leqslant E_{\nu} t^{-\nu}\|u\|_{k}^{\mathrm{L}}+2 \nu
$$

we find that the derivatives of order $\bar{k}+2 \nu$ of $e^{i t R(x)} g(x)$ grow like $t^{\bar{k}+2 \nu}$, so (3.1.10) gives us an estimate $\left|J(t)-S_{\nu}(t)\right| \leqslant D_{\nu} t^{\vec{k}+\nu}$, which is no good because $\bar{k}+\nu$ does not go to $-\infty$ as $\nu \rightarrow \infty$.

Actually, it is not surprising that this approach does not yet work, since it remains to use the essential assumptions that $R(x)$ vanishes to third order at the origin and that $Q(x)+R(x)$ has no critical points outside the origin. Roughly speaking, since the integral (3.1.1) looks "mainly" at the behavior of $u$ near the origin, the degenerate behavior of $R(x)$ at the origin might be expected to overcome the presence of the high powers of $t$ in its derivatives. Of course, we must look at smaller and smaller neighborhoods of the origin as $t \rightarrow \infty$.

To implement these observations, we choose a cutoff function $\mathcal{X}(x)$ which is 1 near the origin and supported in the unit ball, and we split $I(t)$ as the sum $I_{1}(t)+I_{2}(t)$, where

$$
I_{1}(t)=\int e^{i t Q(x)} \mathcal{X}\left(t^{\lambda} x\right) e^{i t R(x)} g(x) d x
$$


and

$$
I_{2}(t)=\int e^{i t Q(x)}\left(1-\mathcal{X}\left(t^{\lambda} x\right)\right) e^{i \ell R(x)} g(x) d x,
$$

where the exponent $\lambda$ is to be chosen later. We form the usual asymptotic expansion for $I_{1}(t)$; since $\mathcal{X}(x) \equiv 1$ near the origin, its presence does not affect the terms of this expansion. In analyzing the error terms, we use the fact that $|x|<t^{-\lambda}$ on the support of $\mathcal{X}\left(t^{\lambda} x\right)$. It turns out that this fact, combined with the third-order vanishing of $R$ at the origin, sufficiently moderates the $L^{1}$ norms of the derivatives of $e^{i t R(x)}$ provided that $\lambda>1 / 4$. On the other hand, we must make $\lambda<1 / 2$, or else the derivatives of $\mathcal{X}\left(t^{\lambda} x\right)$ will grow too rapidly. With these two constraints on $\lambda$, we find that the $S_{\nu}(t)$ 's do give an asymptotic expansion for $I_{1}(t)$. Now we rewrite $I_{2}(t)$ as

$$
\int e^{i t f(x)}\left(1-\mathcal{X}\left(t^{\lambda} x\right)\right) g(x) d x
$$

Since $f(x)$ has no critical points on the supports of $\left[1-\mathcal{X}\left(t^{\lambda} x\right)\right] g(x)$, we can try to integrate by parts with respect to $x$ in order to show that $I_{2}(t)=$ $O\left(t^{-\infty}\right)$. In fact, if $\lambda<1 / 2$, this procedure works and so the asymptotic expansion for $I_{1}(t)$ is also an expansion for $I(t)$.

3.2. Local calculation of the symbol. We begin by recalling the local definition of Fourier integral distributions, following [6]. Let $\mathcal{V}$ be an open subset of $\mathbf{R}^{n}, \Phi(x, \theta)$ a real valued function on $\mathcal{V} \times \mathbf{R}^{N}$ which is positively homogeneous with respect to the $\theta$ variable. $\Phi$ is called a phase function over $\checkmark$ if, when restricted to $\mathscr{V} \times \dot{\mathbf{R}}^{N}\left(\dot{\mathbf{R}}^{N}=\left\{\theta \in \mathbf{R}^{N} \mid \theta \neq 0\right\}\right)$, it is $C^{\infty}$ and has no critical points. An integral of the form

$$
g(x)=\int e^{i \Phi(x, \theta)} a(x, \theta) d \theta
$$

defines $g(x)$ as a smooth function of $x$ if the support of $a$ has a proper projection onto $\mathcal{V}$. It is shown in [6] that (3.2.1) defines $g(x)$ as a distribution on $\mathcal{V}$ as long as $a$ belongs to one of the symbol classes $S^{\mu}\left(\mathcal{V} \times \dot{\mathbf{R}}^{N}\right)$ defined as follows: The complex-valued function $a(x, \theta)$ belongs to $S^{\mu}\left(\mathcal{V} \times \dot{\mathbf{R}}^{N}\right)$ and is called a symbol of order $\mu$ if it is $C^{\infty}$ and if, for any compact subset $K$ of $\mathcal{V}$ and any multi-indices $\alpha$ and $\beta$, there is a constant $C_{\alpha, \beta, K}$ such that

$$
\left|(\partial / \partial x)^{\beta}(\partial / \partial \theta)^{\alpha} a(x, \theta)\right| \leqslant C_{\alpha, \beta, K}(1+|\xi|)^{\mu-|\alpha|}
$$

on $K \times \dot{\mathbf{R}}^{N}$. Specifically, for a test function $u \in \mathscr{D}(\mathfrak{V})$, the integral

$$
\langle g, u\rangle=\int e^{i \Phi(x, \theta)} a(x, \theta) u(x) d x d \theta
$$

may be defined as a limit

$$
\lim _{\varepsilon \rightarrow 0} \int e^{i \Phi(x, \theta)} a(x, \theta) u(x) \mathcal{X}(\varepsilon \theta) d x d \theta
$$


where $\mathcal{X}(\theta)$ is 1 near $\theta=0$ and has compact support; the existence of the limit in (3.2.2) is proven by integrating by parts, using the fact that $\Phi(x, \theta)$ has no critical points, to reduce to the case where $\mu \ll 0$ so that the integral in (3.2.2) is absolutely convergent. This proof gives another definition for $\langle g, u\rangle$ and shows that it is independent of the choice of $\mathfrak{X}$.

It is known further that the singularities of $g$ depend only on the behavior of $a$ and $\Phi$ in a neighborhood of the fibre-critical set

$$
\Sigma_{\Phi}=\left\{(x, \theta) \mid \partial \Phi / \partial \theta_{i}(x, \theta)=0, \theta \neq 0 ; i=1, \ldots, N\right\}
$$

and that the wave front set of $g$ is contained in the image $\Lambda_{\Phi} \subseteq \checkmark \times \dot{\mathbf{R}}^{n^{*}}$ of $\Sigma_{\Phi}$ under the mapping

$$
\lambda_{\Phi}: \Sigma_{\Phi} \rightarrow \widetilde{ } \times \dot{\mathbf{R}}^{n^{*}}, \quad(x, \theta) \mapsto\left(x, d_{x} \Phi\right)
$$

(i.e. $\lambda_{\Phi}(x, \theta)=(x, \xi)$, where $\left.\xi_{i}=\partial \Phi / \partial x_{i}\right)$.

It follows from Corollary 2.2 .2 that the order and symbol of $g$ at $\left(x_{0}, \xi_{0}\right) \in$ $\Lambda_{\Phi}$ depend only upon the behavior of $a$ and $\Phi$ in a neighborhood of $\lambda_{\Phi}^{-1}\left(x_{0}, \xi_{0}\right) \subseteq \Sigma_{\Phi} \subseteq \mathcal{V} \times \dot{\mathbf{R}}^{N}$. In this section, we will calculate this order and symbol under the following further assumptions:

$\lambda_{\Phi}^{-1}\left(x_{0}, \xi_{0}\right)$ consists of a single point $\left(x_{0}, \theta_{0}\right) \in \Sigma_{\Phi} ;$ the differentials of $\partial \Phi / \partial \theta_{1}, \ldots, \partial \Phi / \partial \theta_{N}$ are linearly independent at $\left(x_{0}, \theta_{0}\right)$.

We will use the invariance properties of Fourier integral distributions and of our principal symbol to simplify the calculations. First of all, we may assume that $x_{0}=0$. Next, we may assume that the number $N$ of $\theta$ variables is the minimum necessary to represent $g$ modulo distributions whose wave front set does not contain $\left(0, \xi_{0}\right)$. By Theorem 3.3.4 and Lemma 3.3.5 of [6], this means that the second partial derivatives $\partial^{2} \Phi / \partial \theta_{i} \partial \theta_{j}$ are all zero at $\left(0, \theta_{0}\right)$. Combining this with assumption (3.2.4(b)), we conclude that the matrix $\partial^{2} \Phi / \partial \theta_{i} \partial x_{j}$ has rank $N$ at $\left(0, \theta_{0}\right)$. It follows that $N \leqslant n$ and that, after making a linear transformation of the $x$-coordinates, we may assume that $\partial^{2} \Phi / \partial \theta_{i} \partial x_{j}\left(0, \theta_{0}\right)$ is equal to 1 if $1 \leqslant i=j \leqslant N$ and is 0 otherwise. Finally, the function $x \mapsto \Phi\left(x, \theta_{0}\right)$ has nonzero differential at $x=0$, so may change the $x$-coordinates once again, by a diffeomorphism whose differential at 0 is the identity, so that $\Phi\left(x, \theta_{0}\right)$ becomes the linear function $\left\langle\xi_{0}, x\right\rangle$.

To compute the order and symbol of $g$ at $\left(0, \xi_{0}\right)$, we look now at the family $g_{\varphi}^{\tau}$, where $\varphi(x)=\left\langle\xi_{0}, x\right\rangle$.

$$
\left\langle g_{\varphi}^{\tau}, u\right\rangle=\tau^{n / 2} \int e^{i \Phi(x, \theta)} a(x, \theta) e^{-i \tau \varphi(x)} u(\sqrt{\tau} x) d \theta d x,
$$

or, substituting $x / \sqrt{\tau}$ for $x$,

$$
\left\langle g_{\varphi}^{\tau}, u\right\rangle=\int e^{i \Phi(x / \sqrt{\tau}, \theta)} a(x / \sqrt{\tau}, \theta) e^{-i \tau \varphi(x / \sqrt{\tau})} u(x) d \theta d x
$$


Next, we substitute $\tau \theta$ for $\theta$ and use the homogeneity of $\Phi$ to obtain

$$
\left\langle g_{\varphi}^{\tau}, u\right\rangle=\tau^{N} \int e^{i \tau[\Phi(x / \sqrt{\tau}, \theta)-\varphi(x / \sqrt{\tau})]} a(x / \sqrt{\tau}, \tau \theta) u(x) d \theta d x .
$$

The substitution just made, together with the calculations which follow, closely parallel the calculations on pp. 149-154 of [6], which are also aimed at the determination of a principal symbol. (Reviewing the calculation in [6] is recommended as preparation for reading ours.) The presence of the factors of $\sqrt{\tau}$ in (3.2.7) makes our calculation more laborious; in particular, it prevents the application of the Morse lemma to the phase function $\Phi(x / \sqrt{\tau}, \theta)-\varphi(x / \sqrt{\tau})$. We use instead the method suggested in $\S 3.1$. Let $f(x, \theta)=\Phi(x, \theta)-\varphi(x)$. Since $\left(0, \theta_{0}\right) \in \Sigma_{\Phi}$ and $d_{x} \Phi\left(0, \theta_{0}\right)=\xi_{0}, f(x, \theta)$ has a critical point at $\left(0, \theta_{0}\right)$. By assumption (3.2.4(a)), this is the only critical point of $f(x, \theta)$ for which $x=0$. Since $\varphi(x)$ is linear, the second derivatives of $f$ are the same as those of $\Phi$; therefore, we have $\left(\partial^{2} f / \partial \theta_{i} \partial x_{i}\right)\left(0, \theta_{0}\right)=1$ if $1 \leqslant i \leqslant N$, and all the other second partial derivatives of $f$ vanish at $\left(0, \theta_{0}\right)$. In addition, $f\left(x, \theta_{0}\right) \equiv 0$. It is convenient to relabel the variables $x_{1}, \ldots, x_{n}$ as $y_{1}, \ldots, y_{N}, z_{N+1}, \ldots, z_{n}$ and to write $\Psi$ for $\theta-\theta_{0}$; then we have

$$
f(x, \theta)=\sum_{j=1}^{N} y_{j} \Psi_{j}+r(y, z, \Psi)
$$

where $r$ vanishes to third order at $(0,0,0)$ and $r(y, z, 0) \equiv 0$. We now write (3.2.7) as

$$
\begin{aligned}
\left\langle g_{\varphi}^{\tau}, u\right\rangle=\tau^{N} \int e^{i \sqrt{\tau} \Sigma y_{j} \Psi_{j}}\left\{e^{i \tau r(y / \sqrt{\tau}, z / \sqrt{\tau}, \Psi)}\right. & \\
& \left.\cdot a\left(y / \sqrt{\tau}, z / \sqrt{\tau}, \tau \Psi+\tau \theta_{0}\right) u(y, z)\right\} d \Psi d y d z .
\end{aligned}
$$

In what follows, we will treat $z$ as a parameter; the method of stationary phase will be applied to the $(y, \Psi)$ variables. As in $\S 3.1$, we choose a cutoff function $\mathcal{X}(\Psi)$ which is 1 for $|\Psi| \leqslant 1 / 2$ and supported in the unit ball, and we write

$$
\left\langle g_{\varphi}^{\tau}, u\right\rangle=\tau^{N} \int\left(I_{1}^{\tau}(z)+I_{2}^{\tau}(z)\right) d z
$$

where

$$
\begin{array}{r}
I_{1}^{\tau}(z)=\int e^{i \sqrt{\tau} \Sigma_{j} \Psi_{j}}\left\{e^{i \tau r(y / \sqrt{\tau}, z / \sqrt{\tau}, \Psi)} a\left(y / \sqrt{\tau}, z / \sqrt{\tau}, \tau \Psi+\tau \theta_{0}\right)\right. \\
\left.\cdot u(y, z) \mathscr{X}\left(\tau^{1 / 3} \Psi\right)\right\} d \Psi d y
\end{array}
$$

and 


$$
\begin{array}{r}
I_{2}^{\tau}(z)=\int e^{i \sqrt{\tau} \Sigma y_{j} \Psi_{j}}\left\{e^{i \tau r(y / \sqrt{\tau}, z / \sqrt{\tau}, \Psi)} a\left(y / \sqrt{\tau}, z / \sqrt{\tau}, \tau \Psi+\tau \theta_{0}\right)\right. \\
\left.\cdot u(y, z)\left[1-\mathcal{X}\left(\tau^{1 / 3} \Psi\right)\right]\right\} d \Psi d y .
\end{array}
$$

We will begin by obtaining an asymptotic expansion for $I_{1}^{\tau}(z)$; after that, we will show that $\int I_{2}^{\tau}(z) d z$ is rapidly decreasing.

Let $Q(y, \Psi)=\Sigma y_{j} \Psi_{j}, \tilde{Q}(D)=-\Sigma \partial^{2} / \partial y_{j} \partial \Psi_{j}$. The invertible symmetric matrix with which $Q$ is the associated quadratic form has determinant \pm 1 and signature 0; applying formulas (3.1.1) through (3.1.8), we find that

$$
\left|I_{1}^{\tau}(z)-S_{\nu}^{\tau}(z)\right| \leqslant C_{\nu} \tau^{-(N+\nu) / 2}\left\|\tilde{Q}(D)^{\nu} w^{\tau}\right\|_{2 N+2}^{1},
$$

where

and

$$
\begin{aligned}
w^{\tau}(y, z, \Psi)= & e^{i \tau r(y / \sqrt{\tau}, z / \sqrt{\tau}, \Psi)} a\left(y / \sqrt{\tau}, z / \sqrt{\tau}, \tau \Psi+\tau \theta_{0}\right) \\
& \cdot u(y, z) \mathcal{X}\left(\tau^{1 / 3} \Psi\right)
\end{aligned}
$$

$$
S_{\nu}^{\tau}(z)=(2 \pi \sqrt{\tau})^{-N} \sum_{k=0}^{\nu-1}\left\{\frac{1}{k !}\left[\frac{-i \tilde{Q}(D)}{\sqrt{\tau}}\right]^{k} W^{\tau}\right\}(0, z, 0) .
$$

The $2 N+2$ derivatives and the integral in the norm on the right-hand side of (3.2.11) are taken with respect to $(y, \Psi)$. The result is a function of $z$.

Before making the numerous estimates which will be necessary to justify our result, let us see where we are going by assuming that $I_{1}^{\tau}(z)$ is asymptotic to $S_{1}^{\tau}(z)$ and that $I_{2}^{\tau}(z)$ is rapidly decreasing. Then $\left\langle g_{\varphi}^{\tau}, u\right\rangle$ will be asymptotic to

$$
\tau^{N} \int S_{1}^{\tau}(z) d z=\tau^{N}(2 \pi)^{-N} \tau^{-N / 2} \int e^{i \tau r(0, z / \sqrt{\tau}, 0)} a\left(0, z / \sqrt{\tau}, \tau \theta_{0}\right) u(0, z) d z
$$

which is in turn asymptotic to $\tau^{N / 2}(2 \pi)^{-N} a\left(0,0, \tau \theta_{0}\right) \int u(0, z) d z$. Suppose, for simplicity, that $a$ is, modulo lower order terms, homogeneous of order $\mu$ in $\theta$, then we find that $g_{\varphi}^{\tau} \sim \tau^{\mu+N / 2}(2 \pi)^{-N} a\left(\theta_{0}\right) \delta(y)$; i.e. $g$ is homogeneous of order $\mu+N / 2$ at $\left(0, \xi_{0}\right)$, and its homogeneous $\mu+N / 2$-symbol is $(2 \pi)^{-N} a\left(\theta_{0}\right) \delta(y)$. According to Definition 3.2.2 of [6], $\mu+N / 2$ is equal to $m+n / 4$, where $m$ is the order of $g$ in Hörmander's sense.

Before estimating the growth of the right-hand side of (3.2.11), we begin by analyzing the terms in the expansion $S_{\nu}^{\tau}(z)$ itself; i.e. we look at

$$
\begin{aligned}
T_{k}^{\tau}(z)=\tau^{(-N+k) / 2}\left\{[\tilde{Q}(D)]^{k} w^{\tau}\right\}(0, z, 0) \\
=\tau^{(-N+k) / 2}\left\{\left(\Sigma \frac{\partial^{2}}{\partial y_{j} \partial \Psi_{j}}\right)^{k} e^{i \tau r(y / \sqrt{\tau}, z / \sqrt{\tau}, \Psi)}\right. \\
\left.\cdot a\left(\frac{y}{\sqrt{\tau}}, \frac{z}{\sqrt{\tau}}, \tau \Psi+\tau \theta_{0}\right) u(y, z)\right\}(0, z, 0) .
\end{aligned}
$$


(We may neglect $\mathcal{X}$ since it is identically 1 near $y=\Psi=0$.)

The term for $k=0$ is

$$
T_{0}^{\tau}(z)=\tau^{-N / 2} a\left(0, z / \sqrt{\tau}, \tau \theta_{0}\right) u(0, z),
$$

since $r(0, z, 0)=0$. Taylor's formula gives

where

$$
a\left(0, z, \tau \theta_{0}\right)=a\left(0,0, \tau \theta_{0}\right)+\sum z_{j} a_{j}\left(0, z, \tau \theta_{0}\right)
$$

$$
a_{j}\left(0, z, \tau \theta_{0}\right)=\int_{0}^{1} \frac{\partial a}{\partial z_{j}}\left(0, t z, \tau \theta_{0}\right) d t .
$$

Since $a$ is a symbol of order $\mu, a_{j}\left(0, z, \tau \theta_{0}\right)=O\left(\tau^{\mu}\right)$, uniformly in $z$, so we have

$$
T_{0}^{\tau}(z)=\tau^{-N / 2} a\left(0,0, \tau \theta_{0}\right) u(0, z)+O\left(\tau^{-N / 2+\mu-1 / 2}\right)
$$

uniformly in $z$ for bounded sets of $u$ 's.

To estimate $T_{k}^{\tau}(z)$ for $k>1$, we use the following lemma.

LEMMA 3.2.1. For any $k>1$, the order of growth of $T_{k}^{\tau}(z)$ is at most

$$
\max \left(-\frac{N}{2}+\mu-\frac{1}{2},-\frac{N}{2}+\mu-\frac{k}{6}\right)
$$

(uniformly in $z$ for bounded sets of $u$ ).

Proof. The derivative

$$
\left(\Sigma \frac{\partial^{2}}{\partial y_{j} \partial \Psi_{j}}\right)^{k} e^{i \tau r(y / \sqrt{\tau}, z / \sqrt{\tau}, \Psi)} a\left(y / \sqrt{\tau}, z / \sqrt{\tau}, \tau \Psi+\tau \theta_{0}\right) u(y, z)
$$

is a sum of terms, each of which is a product of factors of one of the forms:

$$
\begin{gathered}
e^{i \tau r(y / \sqrt{\tau}, z / \sqrt{\tau}, \Psi)} ; \\
i \tau^{1-|A| / 2} \frac{\partial^{|A|+|B|} r}{\partial y^{A} \partial \Psi^{B}}(y / \sqrt{\tau}, z / \sqrt{\tau}, \Psi) ; \\
\tau^{-|C| / 2+|D|} \frac{\partial^{|C|+|D|} a}{\partial y^{C} \partial \Psi^{D}}\left(y / \sqrt{\tau}, z / \sqrt{\tau}, \tau \Psi+\tau \theta_{0}\right) ; \\
\frac{\partial^{|E|} u}{\partial y^{E}}(y, z) ;
\end{gathered}
$$

where $A, B, C, D, E$ are multi-indices. Each term contains one factor each of types (3.2.16), (3.2.18), and (3.2.19), together with a number from 0 to $2 k$ of factors of type (3.2.17). The factors of type (3.2.16) and (3.2.19) are uniformly bounded in $z$ for bounded sets of $u$, so we may forget about them. The factor of type (3.2.18) is of order at most $\mu-|C| / 2$; we will use only the fact that this is at most $\mu$. The order of a factor of type (3.2.17) depends in a more 
complicated way upon $|A|$ and $|B|$. Using the facts that $r$ vanishes to third order at $(0,0,0)$ and that $r(y, z, 0)$ is identically zero, we may conclude that a factor of type (3.2.17), evaluated at $(0, z, 0)$, is zero if $|B|=0$ and that its order is at most

$$
\begin{array}{ll}
1 / 2, & \text { if }|A|=0 \text { and }|B|=2 \text { or }|A|=1 \text { and }|B| \geqslant 2, \\
1, & \text { if }|A|=0 \text { and }|B| \geqslant 3, \\
0, & \text { otherwise. }
\end{array}
$$

Suppose that a term contains $s$ factors of type (3.2.17), with multi-indices $\left(A_{1}, B_{1}\right), \ldots,\left(A_{s}, B_{s}\right)$. Since we applied the differential operator $\left(\partial^{2} / \partial y_{j} \partial \Psi_{j}\right)^{k}$, we must have $\Sigma\left|A_{j}\right| \leqslant k$ and $\Sigma\left|B_{j}\right| \leqslant k$.

Let $\alpha$ be the number of factors with $B_{j}=2$ and let $\beta$ be the number of factors with $B_{j} \geqslant 3$. Then $2 \alpha+3 \beta \leqslant k$, and the order of the product of these factors is at most $\alpha / 2+\beta$. The maximum value of the linear function $\alpha / 2+\beta$ on the triangle defined by $\alpha \geqslant 0, \beta \geqslant 0,2 \alpha+3 \beta \leqslant k$ must occur at a vertex; it is $k / 3$. Using the fact that $\alpha$ and $\beta$ must be integers, we can improve this estimate to 0 if $k=1$ and $1 / 2$ if $k=2$. Taking into account now the factor of type (3.2.18) and the factor $\tau^{-(N+k) / 2}$ in (3.2.14) gives the estimate

$$
-N / 2-k / 2+\mu+k / 3=-N / 2+\mu-k / 6
$$

for the order of $T_{k}^{\tau}(z)$; it can be improved to $-N / 2+\mu-1 / 2$ if $k=1$ or 2 .

Applying Lemma 3.2.1 and combining formulas (3.2.13), (3.2.14) and (3.2.15) gives

$$
S_{\nu}^{\tau}(z)=\pi^{-N / 2}(2 \pi)^{-N} a\left(0,0, \tau \theta_{0}\right) u(0, z)+O\left(\tau^{-N / 2+\mu-1 / 2}\right)
$$

uniformly in $z$ on bounded sets of $u$ 's.

We turn next to estimating the right-hand side of (3.2.11).

LEMMA 3.2.2. $\left|\left\{D_{y, \Psi}^{2(N+1)}\left(\sum \partial^{2} / \partial y_{j} \partial \Psi_{j}\right)^{y} w^{\tau}\right\}(y, z, \Psi)\right|=O\left(\tau^{m_{2}}\right)$, uniformly in $(y, z, \Psi)$ for bounded sets of $u$, where $m_{\nu}=\nu / 3+2(N+1) / 3+\mu . D_{y, \Psi}^{2(N+1)}$ is a differential operator of order $\leqslant 2(N+1)$ in the $(y, \Psi)$ variables.

Proof. The procedure is like that in Lemma 3.2.1, but more complicated because we are not setting $y$ and $\Psi$ equal to zero. Instead, we use the presence of the factor $\mathfrak{X}\left(\tau^{1 / 3} \Psi\right)$ to get the inequality $|\Psi| \leqslant \tau^{-1 / 3}$ on the support of $w^{\tau}$. As before, the quantity to be estimated is a sum of terms, each of which is a product of factors of one of the forms (3.2.16) through (3.2.19), together with a factor of the form

$$
\tau^{|F| / 3} \frac{\partial^{|F|} \mathcal{X}}{\partial \Psi^{F}}\left(\tau^{1 / 3} \Psi\right)
$$

which has order $|F| / 3$. The orders of the factors of type (3.2.16), (3.2.18) and 
(3.2.19) are as before; the factors of type (3.2.17) must be estimated anew.

We look then, at the function

$$
f_{A, B}^{\tau}(y, z, \Psi)=\tau^{1-|A| / 2} \frac{\partial^{|A|+|B|}}{\partial y^{A} \partial \Psi^{B}}\left(\frac{y}{\sqrt{\tau}}, \frac{z}{\sqrt{\tau}}, \Psi\right)
$$

restricted to the support of $w^{\tau}$; i.e. we assume that $y$ and $z$ are bounded (since $u$ is to be restricted to a bounded set) and $\Psi=O\left(\tau^{-1 / 3}\right)$. For which values of $(A, B)$ can the order of $f_{A, B}^{\tau}$ be positive? First of all, $|A|$ must be 0 or 1 . If $|A|=0$ and $|B|=1$, the derivative $\partial r / \partial \Psi^{B}$ vanishes to second order at the origin; when evaluated at $(y / \sqrt{\tau}, z / \sqrt{\tau}, \Psi)$ it is of order at most $-2 / 3$, since $y / \sqrt{\tau}, z / \sqrt{\tau}, \Psi$ are all at most $O\left(\tau^{-1 / 3}\right)$. Thus the order of $f_{A, B}^{\tau}$ is at most $1-2 / 3=1 / 3$ if $|A|=0$ and $|B|=1$. Proceeding in the same way, we get the following estimates for the order of $f_{A, B}^{\top}$ :

$$
\begin{array}{lll}
1 / 3 & \text { if }|A|=0 & \text { and }|B|=1 ; \\
2 / 3 & \text { if }|A|=0 & \text { and }|B|=2 ; \\
1 & \text { if }|A|=0 & \text { and }|B| \geqslant 3 ; \\
1 / 6 & \text { if }|A|=1 & \text { and }|B|=1 ; \\
1 / 2 & \text { if }|A|=1 & \text { and }|B| \geqslant 2 .
\end{array}
$$

Now we look at an entire term. Suppose that it contains $s$ factors of type (3.2.17) with multi-indices $\left(A_{1}, B_{1}\right), \ldots,\left(A_{s}, B_{s}\right)$. Let $\alpha$ be the number of factors with $B_{i}=1, \beta$ the number of factors with $B_{i}=2, \gamma$ the number of factors with $B_{i} \geqslant 3$, and $\delta$ the value of $|F|$ in the factor of type (3.2.21). Since we are taking $2(N+1)+\nu \Psi$-derivatives, we must have

$$
\alpha+2 \beta+3 \gamma+\delta \leqslant 2(N+1)+\nu .
$$

Looking at the chart (3.2.23), we find that the maximum order of our term is $\alpha / 3+2 \beta / 3+\gamma+\delta / 3+\mu$ which is at most $\nu / 3+2(N+1) / 3+\mu$.

Using Lemma 3.2.2, we see that the norm on the right-hand side of (3.2.11) is of order at most $\nu / 3+2(N+1) / 3+\mu-N / 3$, since the norm is an integral over a region whose volume is $O\left(\tau^{-N / 3}\right)$. The full right-hand side of (3.2.11) is then of order at most $-\nu / 6-N / 6+2 / 3+\mu$, which goes to $-\infty$ as $\nu \rightarrow \infty$. By choosing $\nu$ sufficiently large $(\geqslant 2 N+7)$, we can make this order $\leqslant-N / 2+\mu-1 / 2$. Using this value of $\nu$ and putting together (3.2.11) and (3.2.20), we obtain

$$
I_{1}^{\tau}(z)=\tau^{-N / 2}(2 \pi)^{-N} a\left(0,0, \tau \theta_{0}\right) u(0, z)+O\left(\tau^{-N / 2+\mu-1 / 2}\right),
$$

uniformly in $z$ on bounded sets of $u$ 's.

To complete our asymptotic evaluation of $g_{\varphi}^{\tau}$, we will estimate $\int I_{2}^{\tau}(z) d z$, where $I_{2}^{\tau}$ is defined in (3.2.10). It is convenient to undo some of the transformations which were applied to (3.2.5); doing so, we arrive at the expression 


$$
\begin{aligned}
& \int I_{2}^{\tau}(z) d z \\
& =\tau^{n / 2} \int e^{i \tau[\Phi(y, z, \theta)-\varphi(y, z)]} a(y, z, \tau \theta) \\
& \cdot u(\sqrt{\tau} y, \sqrt{\tau} z)\left[1-\mathcal{X}\left(\tau^{1 / 3}\left(\theta-\theta_{0}\right)\right)\right] d \Psi d y d z .
\end{aligned}
$$

We will denote by $\nabla_{x} h$ the gradient of any function $h$ with respect to the $x$-coordinates; $h$ may depend as well upon some extra variables.

Since the quadratic part at $\left(0,0, \theta_{0}\right)$ of the function

$$
f(y, z, \theta)=\Phi(y, z, \theta)-\varphi(y, z)
$$

is equal to $\sum y_{j}\left(\theta_{j}-\theta_{0 j}\right)$, it follows from the implicit function theorem that there exists a number $C>0$ and a smooth map $(y, \theta)=k(z)$ defined for $|z| \leqslant C$ such that the set of zeros of $\nabla_{(y, \theta)} f$ in $\{(y, z, \theta)|| y|\leqslant C| z \mid, \leqslant C$, $\left.\left|\theta-\theta_{0}\right| \leqslant C\right\}$ coincides with the graph of $k$. Furthermore $k(0)=\left(0, \theta_{0}\right)$, and the first partial derivatives of $k$ vanish at 0 . One may now conclude (as could have been done much more simply if we had $k \equiv 0$ ) the following:

Lemma 3.2.3. Let positive constants $A$ and $B$ be given. For sufficiently large $\tau$, and $(y, z, \theta)$ in the set

$$
S^{\tau}=\left\{(y, z, \theta)|| y\left|\leqslant A \tau^{-1 / 2},\right| z\left|\leqslant A \tau^{-1 / 2}, B \tau^{-1 / 3} \leqslant\right| \theta-\theta_{0} \mid \leqslant C\right\}
$$

we have the estimate $\left|\nabla_{(y, \theta)} f\right|>D \tau^{-1 / 3}$ for some positive $D$.

Proof. It is sufficient to show that $(y, z, \theta) \in S^{\tau}$ is bounded away from the zero set of $\nabla_{(y, \theta)} f$ by a quantity of order $\tau^{-1 / 3}$, i.e. that $|(y, \theta)-k(z)| \geqslant$ $E \tau^{-1 / 3}$ for some $E>0$ and large $\tau$. But this follows from the inequalities defining $S^{\tau}$ together with the fact that $k$ vanishes to second order at $z=0$.

Our argument now mimics, with flourishes, the one on page 152 of [6].

LEMMA 3.2.4. There exists $\varepsilon>0$ such that, for all $(x, y, \theta)$, one of the following inequalities holds:

$$
\begin{gathered}
\left|\nabla_{\theta} \Phi\right|>\varepsilon \\
|y|>\varepsilon \text { or }|z|>\varepsilon \\
\left|\nabla_{(y, z)} f(y, z, \theta)\right|>\varepsilon(1+|\theta|), \\
\left|\theta-\theta_{0}\right|<C .
\end{gathered}
$$

Proof. Suppose the lemma were false. Then we could find a sequence $\left(y^{j}, z^{j}, \theta^{j}\right)$ such that

(i') $\nabla_{\theta} \Phi\left(y^{j}, z^{j}, \theta^{j}\right) \rightarrow 0$

(ii') $\left(y^{j}, z^{j}\right) \rightarrow 0$,

(iii') $\left(1+\left|\theta^{j}\right|\right)^{-1} \nabla_{(y, z)} f\left(y^{j}, z^{j}, \theta^{j}\right) \rightarrow 0$,

(iv') $\left|\theta^{j}-\theta^{0}\right| \geqslant C$. 
The expression in (iii') can be rewritten as

$$
\nabla_{(y, z)} \Phi\left(y^{j}, z^{j},\left(1+\left|\theta^{j}\right|\right)^{-1} \theta^{j}\right)-\left(1+\left|\theta^{j}\right|\right)^{-1} \nabla_{(y, z)} \varphi\left(y^{j}, z^{j}\right) .
$$

If $\left|\theta^{j}\right|$ is very small, this is close to $-\nabla_{(y, z)} \varphi\left(y^{j}, z^{j}\right)$, which is in turn close to $\nabla_{(y, z)} \varphi(0,0)=\xi_{0}$, which is nonzero; we conclude from the limit (iii') that $\theta^{j}$ is bounded away from zero. Similarly, using (i'), (iii'), and the fact that $\nabla_{(y, z, \theta)} \Phi$ is nowhere zero, we find that $\theta^{j}$ must be bounded away from $\infty$. Passing to a subsequence if necessary, we may assume that $\theta^{j}$ approaches a nonzero limit $\overline{\boldsymbol{\theta}}$. (i') (ii') and (iii') imply that

$$
\nabla_{(y, z, \theta)} f(0,0, \bar{\theta})=0 \text {, }
$$

i.e. $\nabla_{\theta} \Phi(0,0, \bar{\theta})=0$ and $\nabla_{(y, z)} \Phi(0,0, \bar{\theta})=\nabla_{(y, z)} \varphi(0,0)=\xi_{0}$. By our assumption (3.2.4(a)), we must have $\bar{\theta}=\theta_{0}$; but this contradicts the inequality $\left|\bar{\theta}-\theta_{0}\right|>C$ which follows from (iv').

Using a "conic partition of unity", we may write $a=a_{1}+a_{2}$, where $a_{1}$ is supported in the set where (3.2.26(ii), (iii) or (iv)) holds and $a_{2}$ is supported in the set where (3.2.26(i)) holds. The Fourier integral distribution

$$
\int e^{i \Phi(x, \theta)} a_{2}(x, \theta) d \theta
$$

is a $C^{\infty}$ function since $a_{2}$ vanishes on a neighborhood of $\Sigma_{\Phi}$, so its order is $-\infty$, and we may assume that $a=a_{1}$, i.e. that it is supported where (3.2.26(ii), (iii) or (iv)) holds.

Choose one more cutoff function $\Omega(\theta)$ which is 1 in a neighborhood of $\theta=\theta_{0}$ and supported in the set where $\left|\theta-\theta_{0}\right|<C$. Then (3.2.25) gives

$$
\int I_{2}^{\tau}(z) d z=J_{1}^{\tau}+J_{2}^{\tau}
$$

where

$$
\begin{aligned}
J_{1}^{\tau}=\tau^{n / 2} \int e^{i \tau[\Phi(y, z, \theta)-\varphi(y, z)]} a(y, z, \tau \theta) u(\sqrt{\tau} y, \sqrt{\tau} z) \\
\cdot\left[1-\mathcal{X}\left(\tau^{1 / 3}\left(\theta-\theta_{0}\right)\right)\right][1-\Omega(\theta)] d \theta d y d z
\end{aligned}
$$

and $J_{2}^{\tau}$ is the same as $J_{1}^{\tau}$ with $1-\Omega$ replaced by $\Omega$. We assume now that $u$ ranges over a bounded set of test functions. Their supports are contained in a set of the form $\{(y, z)|| y|\leqslant A| z \mid, \leqslant A\}$, so for $\tau \geqslant(A / \varepsilon)^{2}$, we have $|y| \leqslant \varepsilon$ and $|z|<\varepsilon$. In the support of the integrands in $J_{1}^{\tau}$ and $J_{2}^{\tau}$, then, inequalities (3.2.26(i)) and (3.2.26(ii)) cannot hold. By the choice of $\Omega$, we find that (3.2.26(iii)) holds on the support of the integrand in $J_{1}^{\tau}$ and that (3.2.26(iv)) holds on the support of the integrand in $J_{2}^{\tau}$.

We may estimate $J_{1}^{\tau}$ just as Hörmander does on p. 152 of [6]. First we note that, for $\tau$ large, $1-\mathcal{X}\left(\tau^{1 / 3}\left(\theta-\theta_{0}\right)\right)$ is identically 1 on the support of $1-\Omega$, so we may ignore the $\mathcal{X}$ factor. Now we may integrate by parts with respect 
to $(y, z)$. Let $M$ be the vector field

$$
-i\left|\nabla_{(y, z)} f\right|^{-2} \nabla_{(y, z)} f
$$

which satisfies $M \cdot f=-i$. The adjoint $L$ of $M$ is then equal to $-M-$ $\operatorname{div} M$; the coefficients of $L$ as well as all their partial derivatives are of order $O\left(1+|\theta|^{-1}\right)$ on the support of the integrand, by (3.2.26(iii)).

We obtain from (3.2.27), for every integer $\nu$,

$$
\begin{aligned}
J_{1}^{\tau}=\tau^{n / 2} & \int e^{i \tau[\Phi(y, z, \theta)-\varphi(y, z)]} \\
& \cdot^{-\nu} L^{\nu}\{a(y, z, \tau \theta) u(\sqrt{\tau} y, \sqrt{\tau} z)[1-\Omega(\theta)]\} d y d z d \theta .
\end{aligned}
$$

The integrand in (3.2.28) can be bounded by a constant times $\tau^{-\nu}(1+$ $|\theta|)^{-\nu}(1+|\tau \theta|)^{\mu} \tau^{\nu / 2}$, which is less than

$$
\tau^{-\nu / 2}(1+|\theta|)^{\mu-\nu}(1+|\tau|)^{\mu} .
$$

For $\nu$ sufficiently large, the factor $(1+|\theta|)^{\mu-\nu}$ makes the integral absolutely convergent and then the factor $\tau^{-\nu / 2}$ makes $J_{1}^{\tau}$ rapidly decreasing, uniformly on bounded sets of $u$ 's.

For $J_{2}^{\tau}$, we may integrate by parts with respect to $(y, \theta)$. This time, we let $M$ be $-i\left|\nabla_{(y, \theta)} f\right|^{-2} \nabla_{(y, \theta)} f, L$ its adjoint. The support of the integrand in $J_{2}^{\tau}$ is contained in $\left\{(y, z, \theta)|| y\left|\leqslant A \tau^{-1 / 2},\right| z\left|\leqslant A \tau^{-1 / 2}, \tau^{-1 / 3} / 2 \leqslant\right| \theta-\theta_{0} \mid<\right.$ $C$ \}. On that set, we have by Lemma 3.2.3 the estimate $\left|\nabla_{(y, \theta)} f\right| \geqslant D \tau^{-1 / 3}$, which gives us an estimate of $O\left(\tau^{\nu / 3}\right)$ for the coefficients of $L^{\nu}$. We have, for every integer $\nu$,

$$
\begin{aligned}
& J_{2}^{\tau}=\tau^{n / 2} \int e^{i \tau[\Phi(y, z, \theta)-\varphi(y, z)]} \tau^{-\nu} L^{\nu}\{a(y, z, \tau \theta) u(\sqrt{\tau} y, \sqrt{\tau} z) \\
&\left.\cdot\left[1-\mathcal{X}\left(\tau^{1 / 3}\left(\theta-\theta_{0}\right)\right)\right] \mathscr{X}(\theta)\right\} d y d \theta d z .
\end{aligned}
$$

The integrand in (3.2.29) can be bounded by a constant times $\tau^{-\nu+\nu / 3+\mu+\nu / 2}$ $=\tau^{\mu-\nu / 6}$. Making $\nu$ arbitrarily large, we see that $J_{2}^{\tau}$ is rapidly decreasing.

We have now shown that $J_{1}^{\tau}$ and $J_{2}^{\tau}$ are rapidly decreasing; by (3.2.27), so is $\int I_{2}^{\tau}(z) d z$. Using this fact, together with (3.2.8) and (3.2.24), we obtain our final result:

$$
\left\langle g_{\varphi}^{\tau}, u\right\rangle=\tau^{N / 2}(2 \pi)^{-N} a\left(0,0, \tau \theta_{0}\right) \int u(0, z) d z+O\left(\tau^{N / 2+\mu-1 / 2}\right),
$$

uniformly on bounded sets of $u$ 's, or

$$
g_{\varphi}^{\tau}=\tau^{N / 2}(2 \pi)^{-N} a\left(0,0, \tau \theta_{0}\right) \delta(y)+O\left(\tau^{N / 2+\mu-1 / 2}\right) .
$$

We summarize the results of this section in the form of a theorem.

THEOREM 3.2.5. Let $g(x)$ be a Fourier integral distribution on $\mathbf{R}^{n},(0, \xi)$ a 
point in its wavefront set. One can choose coordinates $x=(y, z)$ on $\mathbf{R}^{n}$ and $a$ representation

$$
g(y, z)=\int e^{i \Phi(y, z, \theta)} a(y, z, \theta) d \theta
$$

such that there is a unique value $\theta_{0}$ of $\theta$ for which $\Phi(y, z, \theta)-\langle\xi, x\rangle$ has a critical point at $\left(0,0, \theta_{0}\right)$, and such that the quadratic part of $\Phi(y, z, \theta)-$ $\langle\xi, x\rangle$ at this critical point is $\Sigma y_{j}\left(\theta_{j}-\theta_{0 j}\right)$. Let $N$ be the number of $\theta$-variables in this representation, $\mu$ the order of $a$. Then $O_{(0, \xi)}(g) \leqslant N / 2+\mu$, and $\sigma_{(0, \xi)}^{N / 2+\mu}(g)$, evaluated at the 2-jet of $\langle\xi, x\rangle$, is equal to

$$
\tau^{N / 2}(2 \pi)^{-N} a\left(0,0, \tau \theta_{0}\right) \delta(y)+S^{N / 2+\mu-1 / 2}\left(\mathcal{D}^{\prime}\left(\mathbf{R}^{n}\right)\right) .
$$

COROLlary 3.2.6. In the setting of Theorem 3.2.5, suppose that $a(y, z, \theta)$ is homogeneous of order $\mu$ in $\theta$, modulo symbols of order $\mu-1 / 2$. Then $g$ is homogeneous of order $N / 2+\mu$ at $(0, \xi)$, and its homogeneous $N / 2+\mu$ symbol at $(0, \xi)$, evaluated at the class of $\langle\xi, x\rangle$, is equal to $(2 \pi)^{-N} a\left(0,0, \theta_{0}\right) \delta(y)$.

3.3. Geometric description of the symbol. Let $\Lambda \subseteq T^{*} X$ be a conic lagrangian submanifold. We will denote by $J^{m}(X, \Lambda) \subseteq \mathcal{D}^{\prime}(X)$ the space of Fourier integral distributions of order $m$ associated with $\Lambda$. (We reserve Hörmander's notation $I^{m}(X, \Lambda)$ for $1 / 2$-density valued distributions.) In this section, we will give an invariant description of the symbol $\sigma_{\xi}(g)$ for $g \in$ $J^{m}(X, \Lambda)$ and $\xi \in \Lambda$. Specifically, Theorem 3.3.7 states that $\sigma_{\xi}^{m+n / 4}(g)$ lies in a certain subspace of $\mathcal{S}_{\xi}^{m+n / 4}(X)$ which depends only on $T_{\xi} \Lambda$. In Theorem 3.3.8, we show that our symbol, modulo a natural isomorphism whose construction we describe, is equal to the one defined by Hörmander in [6]. The geometric constructions here are closely related to those of Kostant [7] and Guillemin [3] on symplectic spinors, but we do not use any metaplectic structure.

We begin with some symplectic geometry in $T_{\xi}\left(T^{*} X\right)$, using the canonical 2-form $\Omega$ expressed in local coordinates as $\Sigma d \xi_{i} \wedge d x_{i}$. Denote by $\varrho_{\xi}(X)$ the set of lagrangian subspaces of $T_{\xi}\left(T^{*} X\right)$. The geometry of $T^{*} X$ picks out a distinguished "vertical" element of $\mathcal{L}_{\xi}(X)$, namely $T_{\xi}\left(T_{x}^{*} X\right)$. We observed in $\S 1.6$ that the subset $\mathcal{L}_{\xi}^{H}(X) \subseteq \mathcal{L}_{\xi}(X)$ of "horizontals" could be identified with the set $g_{\xi}$ of 2-jets of $C^{\infty}$ functions $\varphi$ on $X$ such that $d \varphi(x)=\xi$. The "difference" between any two elements of $\mathcal{L}_{\xi}^{H}(X)$ can therefore be considered as a homogeneous quadratic function on $T_{x} X$. We will now generalize this difference construction to define a quadratic form $q_{\lambda \mu}$ when $\lambda \in \mathcal{L}_{\xi}^{H}(X)$ and $\mu \in \mathcal{L}_{\xi}(X)$. The domain of $q_{\lambda \mu}$ will be the projection $V_{\mu}$ of $\mu$ into $T_{x} X$.

Let $\left(e_{1}, \ldots, e_{n}\right)$ be a basis of $T_{x} X$ such that $e_{N+1}, \ldots, e_{n}$ is a basis of $V_{\mu}$, $\left(e_{1}^{*}, \ldots, e_{n}^{*}\right)$ the dual basis in $T_{x}^{*} X$. Identifying $T_{x} X$ with $\lambda$ (by projection) and $T_{x}^{*} X$ with $T_{\xi}\left(T_{x}^{*} X\right)$, we obtain a basis $\left(f_{1}, \ldots, f_{n}, g_{1}, \ldots, g_{n}\right)$ of 
$T_{\xi}\left(T^{*} X\right)$ such that $\left(f_{1}, \ldots, f_{n}\right)$ spans $\lambda,\left(g_{1}, \ldots, g_{n}\right)$ spans $T_{\xi}\left(T_{x}^{*} X\right), \Omega\left(f_{j}, f_{k}\right)$ $=\Omega\left(g_{j}, g_{k}\right)=0$, and $\Omega\left(g_{j}, f_{k}\right)=\delta_{j k}$. Since the projection of $\mu$ is spanned by $e_{N+1}, \ldots, e_{n}$, it follows that $\mu$ contains $n-N$ vectors of the form

$$
v_{j}=f_{j}+\sum_{k=1}^{n} a_{j k} g_{k} \quad(j=N+1, \ldots, n)
$$

and that $\mu$ is contained in the space spanned by $f_{N+1}, \ldots, f_{n}, g_{1}, \ldots, g_{n}$. Since the vectors $g_{1}, \ldots, g_{N}$ are $\Omega$-orthogonal to this last space, they are $\Omega$-orthogonal to $\mu$; since $\mu$ is lagrangian, they are contained in $\mu$, so $\left(g_{1}, \ldots, g_{N}, v_{N+1}, \ldots, v_{n}\right)$ is a basis of $\mu$. It follows that the coefficients $a_{j k}$ for $N+1 \leqslant j, k \leqslant n$ are uniquely determined. Finally, the fact that $\Omega\left(v_{j}, v_{k}\right)$ $=0$ implies that $a_{j k}=a_{k j}$ for $N+1 \leqslant j, k \leqslant n$.

Now let $z=z_{N+1} e_{N+1}+\cdots+z_{n} e_{n}$ be an element of $V_{\mu}$, and define $q_{\lambda \mu}(z)$ to be $\frac{1}{2} \sum_{j, k=N+1}^{n} a_{j k} z_{j} z_{k}$. To see that this definition is independent of the coordinates chosen, we may formulate it invariantly. Given $z \in V_{\mu}$, we lift it in any way we please to an element $z^{\prime}$ of $\mu$. [In our coordinates, we get

$$
z^{\prime}=y_{1} g_{1}+\cdots+y_{N} g_{N}+z_{N+1} v_{N+1}+\cdots+z_{n} v_{n}
$$

for some $\left(y_{1}, \ldots, y_{N}\right)$.] Project $z^{\prime}$ into $T_{\xi}\left(T_{x}^{*} X\right)$ along $\lambda$ to get $z^{\prime \prime}$. [In our coordinates,

$$
z^{\prime \prime}=y_{1} g_{1}+\cdots+y_{N} g_{N}+z_{N+1}\left(v_{N+1}-f_{N+1}\right)+\cdots+z_{n}\left(v_{n}-f_{n}\right) .
$$

Finally, define $q_{\lambda \mu}(z)$ to be $\frac{1}{2} \Omega\left(z^{\prime \prime}, z^{\prime}\right)$. [In our coordinates, we get

$$
\begin{aligned}
& \frac{1}{2} \Omega\left(z^{\prime \prime}, z^{\prime}\right)=\frac{1}{2} \Omega( y_{1} g_{1}+\cdots+y_{N} g_{N}+z_{N+1}\left(v_{N+1}-f_{N+1}\right) \\
&+\cdots+z_{n}\left(v_{n}-f_{n}\right), \quad y_{1} g_{1}+\cdots+y_{N} g_{N} \\
&\left.+z_{N+1} v_{N+1}+\cdots+z_{n} v_{n}\right) \\
&= \frac{1}{2} \Omega\left(-z_{N+1} f_{N+1}-\cdots-z_{n} f_{n}, y_{1} g_{1}+\cdots\right. \\
&\left.\quad+y_{N} g_{N}+z_{N+1} v_{N+1}+\cdots+z_{n} v_{n}\right) \\
&=-\frac{1}{2} \sum_{j, k=N+1}^{n} z_{j} z_{k} \Omega\left(f_{j}, v_{k}\right) \\
&=-\frac{1}{2} \sum_{j, k=N+1}^{n} z_{j} z_{k}\left(-a_{k j}\right) \\
&=\frac{1}{2} \sum_{j, k=N+1}^{n} a_{j k} z_{j} z_{k},
\end{aligned}
$$

which agrees with the earlier definition.]

The symmetric bilinear form $B_{\lambda \mu}$ associated with $q_{\lambda \mu}$ is given by

$$
B_{\lambda \mu}(z, w)=\Omega\left(z^{\prime \prime}, w^{\prime}\right) / 2 \text {. }
$$

It follows that the null space of $q_{\lambda \mu}$ consists of those $z \in V_{\mu}$ having a lift $z^{\prime}$ 
such that $z^{\prime \prime}=0$, i.e. such that $z^{\prime} \in \lambda$. Thus we have

LEMMA 3.3.1. The null space of $q_{\lambda \mu}$ is the projection into $V_{\mu}$ of $\lambda \cap \mu$; $\operatorname{dim}(\lambda \cap \mu)$ equals the nullity of $q_{\lambda \mu}$.

Suppose, now, that $\bar{\lambda}$ is a second horizontal space. It has a basis of the form

$$
\overline{f_{j}}=f_{j}+\sum_{k=1}^{n} b_{j k} g_{k}
$$

where $\left(b_{j k}\right)$ is an $n \times n$ symmetric matrix. To compute $q_{\bar{\lambda}_{\mu}}(z)$, we must find vectors of the form

$$
\bar{v}_{j}=\bar{f}_{j}+\sum_{k=1}^{n} \bar{a}_{j k} g_{k} \quad(j=N+1, \ldots, n)
$$

in $\mu$. To do this, we combine (3.3.1) and (3.3.2) to get

$$
v_{j}=\bar{f}_{j}+\sum_{k=1}^{n}\left(a_{j k}-b_{j k}\right) g_{k} \quad(j=N+1, \ldots, n) ;
$$

so we have

$$
q_{\bar{\lambda} \mu}(z)=\frac{1}{2} \sum_{j, k=N+1}^{n}\left(a_{j k}-b_{j k}\right) z_{j} z_{k}=q_{\lambda \mu}(z)-\frac{1}{2} \sum_{j, k=N+1}^{N} b_{j k} z_{j} z_{k} .
$$

Using (3.3.2), we may recognize the last sum as $q_{\lambda \bar{\lambda}}(z)$, so we have the following result.

Lemma 3.3.2. Let $\lambda, \bar{\lambda} \in \mathcal{L}_{\xi}^{H}(X), \mu \in \mathcal{E}_{\xi}(X)$. Then $q_{\lambda \mu}=q_{\lambda \bar{\lambda}}+q_{\bar{\lambda}_{\mu}}$, where $q_{\lambda \bar{\lambda}}$ is understood to be restricted to $V_{\mu}$.

Since any quadratic form on $V_{\mu}$ can be extended to $T_{x} X$, and any quadratic form on $T_{x} X$ can be realized as $q_{\lambda \bar{\lambda}}$ for some $\lambda, \bar{\lambda}$ being fixed, Lemma 3.3.2 has the following consequence.

Corollary 3.3.3. Let $\mu \in \mathcal{L}_{\xi}(X)$. Then there exists an element $\lambda \in \mathcal{L}_{\xi}^{H}(X)$ such that $q_{\lambda \mu}=0$, i.e. such that $\operatorname{dim}(\lambda \cap \mu)=\operatorname{dim} V_{\mu}$. If $\lambda$ and $\bar{\lambda}$ are two such elements, then the restriction to $V_{\mu}$ of $q_{\lambda \bar{\lambda}}$ is identically zero.

We need one more preliminary notion.

Definition 3.3.4. Let $W$ be a vector space $V \subseteq W$ a subspace. A distribution $\delta \in \mathscr{D}^{\prime}(W)$ is called a constant $\delta$-function along $V$ if, for some (and hence every) linear coordinate system $\left(x_{1}, \ldots, x_{n}\right)$ on $W$ such that $V$ is defined by $x_{1}=\cdots=x_{k}=0, \delta$ is a constant multiple of $\delta\left(x_{1}\right) \cdots \delta\left(x_{k}\right)$, i.e.

$$
\langle\delta, u\rangle=c \int_{V} u\left(0, \ldots, 0, x_{k+1}, \ldots, x_{n}\right) d x_{k+1} \cdots d x_{n}
$$

for some complex number $C$. 
The $\delta$-functions along $V$ form a 1-dimensional subspace of $\mathscr{D}^{\prime}(W)$ which we will denote by $\Delta_{V}(W)$. It is invariant under multiplication by $C^{\infty}$ functions on $W$ which are constant along $V$.

Now we recall the definition of $\tilde{\mathscr{D}}_{\xi}^{\prime}(X)$ from $\$ 1$.6. Identifying the 2-jets $g_{\xi}(X)$ with the horizontal lagrangian subspaces $\mathcal{L}_{\xi}^{H}(X)$, we may describe $\tilde{D}_{\xi}^{\prime}(X)$ as the set of maps $\lambda \mapsto g_{\lambda}$ from $\mathcal{L}_{\xi}^{H}(X)$ to $\mathscr{D}^{\prime}\left(T_{x} X\right)$ such that $g_{\bar{\lambda}}=e^{-i q_{\lambda} \bar{g}} g_{\lambda}$ for each $\lambda$ and $\bar{\lambda}$ in $\mathcal{L}_{\xi}^{H}(X)$.

Definition 3.3.5. Let $\mu \in \mathcal{L}_{\xi}(X)$. We define $\tilde{\mathscr{D}}_{\xi, \mu}^{\prime}(X) \subseteq \tilde{\mathscr{D}}_{\xi}^{\prime}(X)$ to consist of those maps $\lambda \mapsto g_{\lambda}$ which assign a constant $\delta$-function along $V_{\mu} \subseteq T_{x} X$ to each $\lambda \in \mathcal{L}_{\xi}^{H}(X)$ for which $\operatorname{dim}(\lambda \cap \mu)=\operatorname{dim} V_{\mu}$.

Corollary 3.3.3 and the remark following Definition 3.3.4 have the following consequence.

LEMMA 3.3.6. For each $\mu \in \mathfrak{E}_{\xi}(X)$, $\tilde{\mathscr{D}}_{\xi, \mu}^{\prime}$ is a 1-dimensional subspace of $\tilde{\mathscr{D}}_{\xi}^{\prime}(X)$.

The symbol space $\delta_{\xi}^{N}(X)$ as defined in $\S 1.6$ is naturally isomorphic to

$$
S^{N}\left(\tilde{\mathscr{D}}_{\xi}^{\prime}(X)\right) / S^{N-1 / 2}\left(\tilde{D}_{\xi}^{\prime}(X)\right) \text {. }
$$

It contains as a subspace $S^{N}\left(\tilde{\mathscr{D}}_{\xi, \mu}^{\prime}(X)\right) / S^{N-1 / 2}\left(\tilde{\mathscr{D}}_{\xi, \mu}^{\prime}(X)\right)$, which we denote by $\mathcal{S}_{\xi, \mu}^{N}(X)$.

TheOREM 3.3.7. Let $\Lambda \subseteq T^{*} X$ be a conic lagrangian submanifold, $g$ a Fourier integral distribution in $J^{m}(X, \Lambda)$.

(i) if $0 \neq \xi \in T^{*} X$, $\xi \notin \Lambda$, then $O_{\xi}(g)=-\infty$,

(ii) if $\xi \in \Lambda$, then $O_{\xi}(g) \leqslant m+n / 4(n=\operatorname{dim} X)$

and $\sigma_{\xi}^{m+n / 4}(g) \in \mathcal{S}_{\xi, \mu}^{m+n / 4}(X)$ where $\mu=T_{\xi} \Lambda$. In particular, if $g$ has homogeneous principal symbol in the sense of [6], then the homogeneous $m+n / 4$ symbol of $g$ lies in the 1-dimensional space $\tilde{D}_{\xi, \mu}^{\prime}(X)$.

Proof. Statement (i) follows from Corollary 2.2.2 and the fact (Proposition 2.5.7 of [6]) that $\xi \notin \mathrm{WF}(g)$.

To prove statement (ii), we can assume ourselves to be in the situation of Theorem 3.2.5. In fact, we may write $g=g_{1}+g_{2}$, where $\xi \notin \mathrm{WF}\left(g_{1}\right)$ and $g_{2}$ is as described in Theorem 3.2.5. By Corollary 2.2.2, $g_{1}$ does not contribute to the order and symbol of $g$ at $\xi$, so we may assume that $g$ itself is in the form given by Theorem 3.2.5. We will now use the coordinates, phase function, and amplitude of that theorem.

We assume, then, that $X=\mathbf{R}^{n}, \xi=(0, \xi)$ and $\Lambda=\Lambda_{\Phi}$, where $\Phi$ has the properties enunciated in the statement of Theorem 3.2.5. First of all, according to the definition of $I^{m}(X, \Lambda)([6$, Definition 3.2.2]) the order of the amplitude $a$ must be $\mu=m+n / 4-N / 2$. By Theorem 3.2.5, we have $O_{(0, \xi)}(g)<N / 2+\mu=m+n / 4$.

To prove that the symbol as given by Theorem 3.2.5 lies in $\mathcal{S}_{(0, \xi), \mu}^{N}\left(\mathbf{R}^{n}\right)$, 
where $\mu=T_{(0, \xi)} \Lambda_{\Phi}$, it suffices to show that the factor $\delta(y)$ represents an element of $\mathscr{D}_{(0, \xi), \mu}^{\prime}\left(\mathbf{R}^{n}\right)$. To do this, we must determine what $\mu$ is. We use the coordinates $\left(y_{1}, \ldots, y_{N}, z_{N+1}, \ldots, z_{n}\right)$ on $\mathbf{R}^{n}$ and dual coordinates $\left(\eta_{1}, \ldots, \eta_{N}, \zeta_{N+1}, \ldots, \zeta_{n}\right)$ on $\mathbf{R}^{n *}$. Writing $\left(\eta_{0}, \zeta_{0}\right)$ for $\xi$, we have, modulo functions vanishing to order $\geqslant 3$ at $\left(0,0, \theta_{0}\right)$,

$$
\Phi(y, z, \theta)=\Sigma \eta_{0 j} y_{j}+\Sigma \zeta_{0 j}^{0} z_{j}+\Sigma\left(\theta_{j}-\theta_{0 j}\right) y_{j} \text {. }
$$

The terms of order $\geqslant 3$ in $\Phi$ will not effect the computation of $T_{(0, \xi)}\left(\Lambda_{\Phi}\right)$, so we may compute by pretending that $\Phi$ is given precisely by (3.3.3).

We have $\partial \Phi / \partial \theta_{j}(y, z, \theta)=y_{j}$, so $\Sigma_{\Phi}$ is $\{(0, z, \theta) \mid \theta \neq 0\}$. Now $\Lambda_{\Phi}$ is defined by $\eta_{j}=\partial \Phi / \partial y_{j}(0, z, \theta)$ and $\zeta_{j}=\partial \Phi / \partial z_{j}(0, z, \theta)$; i.e. $\eta_{j}=\eta_{0 j}+\left(\theta_{j}-\right.$ $\left.\theta_{0 j}\right)$ and $\zeta_{j}=\zeta_{0 j}$. The tangent space $\mu=T_{(0, \xi)} \Lambda_{\Phi}$ has as a basis, therefore, the vectors $\left(\partial / \partial \eta_{1}, \ldots, \partial / \partial \eta_{N}, \partial / \partial z_{N+1}, \ldots, \partial / \partial z_{n}\right)$. The projection $V_{\mu}$ into $T_{0} \mathbf{R}^{n}$ has basis $\left(\partial / \partial z_{N+1}, \ldots, \partial / \partial z_{n}\right)$ and dimension $n-N$. Identifying $T_{0} \mathbf{R}^{n}$ with $\mathbf{R}^{n}$, we find that a constant $\delta$-function along $V_{\mu}$ is simply a constant multiple of $\delta(y)$.

We will be finished if we can show that the element $\lambda$ of $\mathcal{L}_{(0, \xi)}^{H}\left(\mathbf{R}^{n}\right)$ at which the symbol has been evaluated in Theorem 3.2.5 is in the special class involved in Definition 3.3.5; i.e. we must $\operatorname{show} \operatorname{dim}(\lambda \cap \mu)=n-N$. $\lambda$ is just the tangent space at $\left(0, \eta_{0}, \zeta_{0}\right)$ of the image $d \varphi\left(\mathbf{R}^{n}\right)$ of the differential of the function $\varphi(y, z)=\langle\xi, x\rangle=\Sigma \eta_{0 i} y_{i}+\Sigma \zeta_{0 i} z_{i}$. Then $d \varphi\left(\mathbf{R}^{n}\right)$ is simply $\left\{\left(y, z, \eta_{0}, \zeta_{0}\right)\right\}$, and its tangent space $\lambda$ at $\left(0,0, \eta_{0}, \zeta_{0}\right)$ is spanned by $\left(\partial / \partial y_{1}, \ldots, \partial / \partial y_{N}, \partial / \partial z_{N+1}, \ldots, \partial / \partial z_{n}\right)$. Comparing this with the basis for $\mu$ given above, we find that the dimension of $\lambda \cap \mu$ is $n-N$.

Finally, the statement about homogeneous principal symbols follows from Corollary 3.2.6.

In the rest of this section, we will relate our symbol to that of Hörmander. To begin, we will identify the space $\Delta_{V_{\mu}}\left(T_{x} X\right)$ of constant $\delta$-functions along $V_{\mu} \subseteq T_{x} X$ with a space defined in terms of $\mu$ rather than $V_{\mu}$. The "test functions" for $\mathscr{D}^{\prime}\left(T_{x} X\right)$ have values in the space $\Omega_{1}\left(T_{x} X\right)$ of 1-densitites on $T_{x} X$. (See $\S 1.1$ of [2].) To apply an element of $\Delta_{V_{u}}\left(T_{x} X\right)$ to such a test function, we should integrate it over $V_{\mu}$, but we can do so only if has values in $\Omega_{1}\left(V_{\mu}\right)$. Specification of a constant $\delta$-function along $V_{\mu}$ amounts, therefore, to specifying a linear map from $\Omega_{1}\left(T_{x} X\right)$ to $\Omega_{1}\left(V_{\mu}\right)$. In other words, we have a natural isomorphism

$$
\Delta_{V_{\mu}}\left(T_{x} X\right) \approx \Omega_{-1}\left(T_{x} X\right) \otimes \Omega_{1}\left(V_{\mu}\right)
$$

Next we wish to analyze $\Omega_{1}\left(V_{\mu}\right)$. The kernel of the projection of $\mu$ onto $V_{\mu}$ is a subspace of $T_{\xi}\left(T_{x}^{*} X\right)$; when we identify $T_{\xi}\left(T_{x}^{*} X\right)$ with $T_{x}^{*} X$, the kernel of this projection becomes identified with the annihilator $V_{\mu}^{\perp} \subseteq T_{x}^{*} X$, which may in turn be identified with $\left(T_{x} X / V_{\mu}\right)^{*}$. Thus, we have an exact sequence

$$
0 \rightarrow\left(T_{x} X / V_{\mu}\right)^{*} \rightarrow \mu \rightarrow V_{\mu} \rightarrow 0
$$


inducing for each $\alpha$ an isomorphism $\Omega_{\alpha}(\mu) \approx \Omega_{\alpha}\left(T_{x} X / V_{\mu}\right)^{*} \otimes \Omega_{\alpha}\left(V_{\mu}\right)$. Now

$$
\Omega_{\alpha}\left(T_{x} X / V_{\mu}\right)^{*} \approx \Omega_{-\alpha}\left(T_{x} X / V_{\mu}\right) \approx \Omega_{-\alpha}\left(T_{x} X\right) \otimes \Omega_{\alpha}\left(V_{\mu}\right),
$$

again by natural isomorphisms, so we have

$$
\Omega_{\alpha}(\mu) \approx \Omega_{2 \alpha}\left(V_{\mu}\right) \otimes \Omega_{-\alpha}\left(T_{x} X\right),
$$

or

$$
\Omega_{2 \alpha}\left(V_{\mu}\right) \approx \Omega_{\alpha}(\mu) \otimes \Omega_{\alpha}\left(T_{x} X\right) .
$$

To combine this with (3.3.4) in order to eliminate $V_{\mu}$, we must take $\alpha=1 / 2$, in which case we obtain

$$
\Delta_{V_{\mu}}\left(T_{x} X\right) \approx \Omega_{1 / 2}(\mu) \otimes \Omega_{-1 / 2}\left(T_{x} X\right) .
$$

Notice that although we have been working with ordinary distributions (i.e. generalized functions), our geometric considerations have forced 1/2-densities upon us.

Definition 3.3.5 gives us a natural isomorphism

$$
\tilde{\mathbb{D}}_{\xi, \mu}^{\prime}(X) \approx \Delta_{V_{\mu}}\left(T_{x} X\right)
$$

which is independent of the choice of $\lambda \in \mathcal{C}_{\xi}^{H}(X)$ with $\operatorname{dim}(\lambda \cap \mu)=\operatorname{dim} V_{\mu}$. Combining (3.3.5) and (3.3.6) gives us a natural isomorphism

$$
\tilde{\mathscr{D}}_{\xi, \mu}^{\prime}(X) \approx \Omega_{1 / 2}(\mu) \otimes \Omega_{-1 / 2}\left(T_{x} X\right),
$$

so that the symbol $\sigma_{\xi}^{m+n / 4}(g)$ may be considered as lying in

$$
S^{m+n / 4}\left(\Omega_{1 / 2}(\mu) \otimes \Omega_{-1 / 2}\left(T_{x} X\right)\right) / S^{m+n / 4-1 / 2}\left(\Omega_{1 / 2}(\mu) \otimes \Omega_{-1 / 2}\left(T_{x} X\right)\right) .
$$

Using the coordinates of Theorems 3.2.5 and 3.3.7 and following through all the natural isomorphisms described above we find the symbol to be

$$
\begin{gathered}
\sigma_{\xi}^{m+n / 4}(g)=\tau^{N / 2}(2 \pi)^{-N} a\left(0,0, \tau \theta_{0}\right)\left|d \eta_{1} \cdots d \eta_{N} d z_{N+1} \cdots d z_{n}\right|^{1 / 2} \\
\otimes\left|d_{y_{1}} \cdots d y_{N} d z_{N+1} \cdots d z_{n}\right|^{-1 / 2} .
\end{gathered}
$$

To compare our symbol with Hörmander's, we must first take into account the fact that Hörmander is working with 1/2-density distributions. But either symbol calculus can easily be extended to deal with vector-bundle-valued Fourier integral distributions. If $E$ is any complex vector bundle over $X$, we may define $J^{m}(X, \Lambda ; E)$ to be

$$
J^{m}(X, \Lambda) \otimes E \subseteq \mathscr{D}^{\prime}(X) \otimes E=\mathscr{D}^{\prime}(X ; E) ;
$$

any element of $J^{m}(X, \Lambda ; E)$ is thus locally a sum of products of elements of $J^{m}(X, \Lambda)$ with smooth sections of $E$. With respect to a local trivialization of $E$, an element of $J^{m}(X, \Lambda ; E)$ is just a $\operatorname{dim} E$-tuple of elements of $J^{m}(X, \Lambda)$, so Theorem 3.3.7 remains true if we replace $J^{m}(X, \Lambda)$ by $J^{m}(X, \Lambda ; E)$, $\tilde{\mathscr{D}}_{\xi, \mu}^{\prime}(X)$ by $\tilde{\mathcal{D}}_{\xi, \mu}^{\prime}(X, E)=\tilde{\mathscr{D}}_{\xi, \mu}^{\prime}(X) \otimes E_{x}$, and $\mathcal{S}_{\xi, \mu}^{m+n / 4}(X)$ by $\mathcal{S}_{\xi, \mu}^{m+n / 4}(X ; E)=$ $\mathcal{S}_{\xi, \mu}^{m+n / 4}(X) \otimes E$. 
If we take $E$ to be $\Omega_{1 / 2}(T X),(3.3 .7)$ becomes $\tilde{\mathscr{D}}_{\xi, \mu}\left(X ; \Omega_{1 / 2}(T X)\right) \approx \Omega_{1 / 2}(\mu)$. Hörmander's space $I^{m}(X, \Lambda)$ is just our $J^{m}\left(X, \Lambda ; \Omega_{1 / 2}(T X)\right)$. If we compute the symbol of the distribution $h$ which is, in our local coordinates,

\section{(3.3.8) becomes}

$$
g\left|d y_{1} \cdots d y_{N} d z_{N+1} \cdots d z_{n}\right|^{1 / 2} \text {, }
$$

$$
\sigma_{\xi}^{m+n / 4}(h)=\tau^{N / 2}(2 \pi)^{-N} a\left(0,0, \tau \theta_{0}\right)\left|d \eta_{1} \cdots d \eta_{N} d z_{N+1} \cdots d z_{n}\right|^{1 / 2} .
$$

If $a$ is homogeneous (modulo lower order terms), the homogeneous $m+n / 4$ symbol of $h$ at $\xi$ is

$$
(2 \pi)^{-N} a\left(0,0, \theta_{0}\right)\left|d \eta_{1} \cdots d \eta_{N} d z_{N+1} \cdots d z_{n}\right|^{1 / 2} \in \Omega_{1 / 2}(\mu) .
$$

To compare our symbol with Hörmander's, we will treat first this homogeneous case, for it is only there that Hörmander's symbol is well defined at points of $T^{*} X$. (In general, it is defined as a function on each ray of $T_{*} X$, modulo functions of lower growth order.) Now Hörmander's symbol lies in the tensor product $\Omega_{1 / 2}(\mu) \otimes L_{\mu}$, where $L_{\mu}$ is the fibre over $\xi$ of the flat line bundle $L$ defined on p. 148 of [6]. (Theorem 3.3.3 of [6] shows that $L_{\mu}$ can be defined in terms of $\mu$ alone.) To compare the two symbols, we must identify $L_{\mu}$ with $\mathbf{C}$. By the original definition of $L$, such an identification arises every time we choose a phase function for $\Lambda$ near $\xi$. But there is a natural (up to equivalence) choice for a phase function near $\xi$ the "minimal" one, in which the number of phase variables is equal to the dimension of $T_{\xi} \Lambda \cap T_{\xi}\left(T_{x}^{*} X\right)$. This gives us a natural identification of $L_{\mu}$ with $\mathbf{C}$ and, hence, of $\Omega_{1 / 2}(\mu) \otimes$ $L_{\mu}$ with $\Omega_{1 / 2}(\mu)$. (This natural trivialization of each fibre of $L$ does not define a trivialization of $L$ itself because it is not continuous from fibre to fibre.)

Using Definition 3.2.2 with the convention mentioned after Theorem 3.3.4 and the construction on p. 148 of [6], we find that Hörmander's symbol for $h$ at $\xi$ has the value

$$
(2 \pi)^{n / 4-N / 2} a\left(0,0, \theta_{0}\right) \omega,
$$

where $\omega$ is the pullback to $\mu$ of a $1 / 2$-density on $T_{\left(0,0, \theta_{0}\right)} \Sigma_{\Phi}$ which Hörmander denotes by " $\sqrt{d_{c}}$ ". Because of the special form in our coordinates of the quadratic part of our phase function, all the Jacobian determinants involved in the definition of $d_{C}$ and $\omega$ are equal to \pm 1 , and $\omega$ is equal to $\left|d \eta_{1} \cdots d \eta_{N} d z_{N+1} \cdots d z_{n}\right|^{1 / 2}$.

Comparing (3.3.10) and (3.3.11) we find that if we define an isomorphism

$$
\tilde{\mathscr{D}}_{\xi, \mu}^{\prime}\left(X ; \Omega_{1 / 2}(T X)\right) \stackrel{\iota^{\mu}}{\rightarrow} \Omega_{1 / 2}(\mu) \otimes L_{\mu}
$$

by combining all the isomorphisms already mentioned with a multiplication by $(2 \pi)^{n / 4+N / 2}$, then the image under $\iota_{\mu}$ of our symbol is equal to Hörmander's. 
To handle the nonhomogeneous case, we must look at Hörmander's symbol along the ray $\{\tau \xi\}$ as $\tau \rightarrow \infty$. If we denote by $\tau \mu$ the image of $\mu$ under the differential at $\xi$ of multiplication by $\tau$ from $T^{*} X$ to $T^{*} X$, then there is a natural pullback $\Omega_{1 / 2}(\tau \mu) \rightarrow \Omega_{1 / 2}(\mu)$ which maps $\left|d \eta_{1} \cdots d \eta_{N} d z_{N+1} \cdots d z_{n}\right|^{1 / 2}$ at $\tau \mu$ to $\tau^{N / 2}\left|d \eta_{1} \cdots d \eta_{N} d z_{N+1} \cdots d z_{n}\right|^{1 / 2}$. This factor of $\tau^{N / 2}$ matches exactly the factor of $\tau^{N / 2}$ in (3.3.9), and we have the following result.

THEOREM 3.3.8. Let $\xi \in T_{x}^{*} X$ be a cotangent vector, $\mu \subseteq T_{\xi}\left(T^{*} X\right) a$ lagrangian subspace. There is an isomorphism $\iota_{\mu}$ from the space $\tilde{D}_{\xi, \mu}^{\prime}\left(X ; \Omega_{1 / 2}(T X)\right)$ of "twisted constant $\delta$-functions along the projection $V_{\mu} \subseteq$ $T_{x} X$ with values in $\Omega_{1 / 2}\left(T_{x} X\right)$ " to Hörmander's symbol space $\Omega_{1 / 2}(\mu) \otimes L_{\mu} ; \iota_{\mu}$ transforms symbols in the following sense.

Let $\Lambda \subseteq T^{*} X$ be a conic lagrangian submanifold containing $\xi$ with $T_{\xi} \Lambda=\mu$, $h$ a Fourier integral distribution in $I^{m}(X, \Lambda) \subseteq \mathscr{D}^{\prime}\left(X ; \Omega_{1 / 2}(T X)\right)$. For each $\tau>0$, let $a_{\tau \xi}(h) \in \Omega_{1 / 2}(\tau \mu) \otimes L_{\tau \mu}$ be the value at $\tau \xi$ of Hörmander's principal symbol for $h\left(\tau \mu=T_{\tau \xi} \Lambda\right)$. Then $O_{\xi}(h) \leqslant m+n / 4$, and

$$
\sigma_{\xi}^{m+n / 4}(h)^{\tau} \equiv\left(\iota_{\mu}^{-1} m^{\tau}\left(a_{\tau \xi}(h)\right)\right) \quad\left(\text { modulo } O\left(\tau^{m+n / 4-1 / 2}\right)\right)
$$

where

$$
m^{\tau}: \Omega_{1 / 2}(\tau \mu) \otimes L_{\tau \mu} \rightarrow \Omega_{1 / 2}(\mu) \otimes L_{\mu}
$$

is the pullback map induced by multiplication by $\tau$.

$h$ is homogeneous of order $m+n / 4$ at $\xi$ if and only if $a(h)$ is homogeneous in the sense that $m^{\tau} a_{\tau \xi}(h)=\tau^{m+n / 4} a_{\xi}(h)$; in this case, the homogeneous $m+n / 4$ symbol of $h$ at $\xi$ is equal to $\iota_{\mu}^{-1}\left(a_{\xi}(h)\right)$.

Theorem 3.3.8 has a geometric complement, the details of which are presented elsewhere [13]. The spaces $\tilde{D}_{\xi}^{\prime}(X ; E)$ can be considered as the fibres of a smooth, infinite-dimensional vector bundle $\tilde{\mathscr{D}}^{\prime}(X, E)$ over $T^{*} X$. Over $T^{*} X$, we also have the "lagrangian grassmannian" bundle $\mathscr{L}(X)$ $\stackrel{l}{\rightarrow} T^{*} X$ whose fibre over $\xi$ is the manifold $\mathcal{E}_{\xi}(X)$ of lagrangian subspaces of $T_{\xi}\left(T^{*} X\right)$. The space $\tilde{D}_{\xi, \mu}^{\prime}(X ; E)$ is a subspace of the fibre at $\mu$ of the pulled back bundle $l^{*}\left(\tilde{\mathscr{D}}^{\prime}(X ; E)\right)$. In [13], we show that the spaces $\tilde{\mathscr{D}} \tilde{\mathcal{D}}_{\xi, \mu}^{\prime}(X ; E)$ form a smooth subbundle of $\left.l^{*}(\tilde{\mathscr{D}})^{\prime}(X ; E)\right)$, and that the maps $\iota_{\mu}$ give a smooth bundle isomorphism from $\left\{\tilde{\mathscr{D}}_{\xi, \mu}^{\prime}\left(X ; \Omega_{1 / 2}(T X)\right)\right\}_{\mu \in \mathcal{E}(X)}$ to Hörmander's "universal symbol bundle" $\left\{\Omega_{1 / 2}(\mu) \otimes L_{\mu}\right\}_{\mu \in \mathcal{E}(X)}$. We can consider the spaces $\tilde{\mathscr{D}}_{\xi, \mu}^{\prime}\left(X ; \Omega_{1 / 2}(T X)\right)$ as giving a new realization of Hörmander's symbol bundle.

\section{REFERENCES}

1. A. P. Calderon, Calcul précisé sur les opérateurs intégraux-singuliers, Cours professé à la faculté des sciences de Paris, notes par Marc Durand, 1966. 
2. J. J. Duistermaat, Fourier integral operators, New York Univ., 1973.

3. V. Guillemin, Symplectic spinors and partial differential equations, Colloque de Géométrie Sympléctique et Physique Mathématique, C.N.R.S., Paris, 1976.

4. L. Hörmander, Linear partial differential operators, Springer-Verlag, Berlin, 1963. MR 28 \#4221.

5. Pseudo-differential operators and non-elliptic boundary problems, Ann. of Math. (2) 83 (1966), 129-209. MR 38 \#1387.

5a. __, Pseudo-differential operators and hypoelliptic equations, Proc. Sympos. Pure Math., vol. 10, Amer. Math. Soc., Providence, R.I., 1967, pp. 138-183. MR 52 \# 4033.

6. __ Fourier integral operators. I, Acta Math. 127 (1971), 79-183. MR 52 \# 9299.

7. B. Kostant, Symplectic spinors, Symposia Math. 14 (1974), pp. 139-152. MR 53 \#4139.

8. S. Kojasiewicz, Sur la valeur et la limite d'une distribution en un point, Studia Math. 16 (1957), 1-36. MR 19, 433.

9. 21 \# 5892.

10. A. Melin, Lower bounds for pseudo-differential operators, Ark. Math. 9 (1971), 117-140. MR 48 \#6735.

11. L. Schwartz, Theorie des distributions, Publ. Inst. Math. Univ. Strasbourg, nos. 9-10, Hermann, Paris, 1966. MR 35 \#730.

12. A. Weinstein, The principal symbol of a distribution, Bull. Amer. Math. Soc. 82(1976), 548-550.

13. , Lectures on sympletic manifolds, CBMS Regional Conf. Ser. in Math., vol. 29, Amer. Math. Soc., Providence, R. I., 1977.

Institut des Hautes Etudes Scientifiques, 35, route de Chartres, 91440-Bures S/YvetTe, France.

Current address: Department of Mathematics, University of California, Berkeley, California 94720 